\title{
DMSO as a Methine Source in TFA-Mediated One-Pot Tandem Regioselective Synthesis of 3-Substituted-1-Aryl- 1H-Pyrazolo-[3,4-b]quinolines from Anilines and Pyrazolones
}

Pushpendra Yadav, ${ }^{\dagger, *}$, Annapurna Awasthi, ${ }^{\dagger}, *$, Sabapathi Gokulnath, ${ }^{\S}$ and Dharmendra Kumar Tiwari ${ }^{*+, t}$

'Molecular Synthesis and Drug Discovery Laboratory, Center of Biomedical Research (CBMR), Sanjay Gandhi Post-Graduate Institute of Medical Sciences Campus, Raebareli Road, Lucknow, 226014, UP, India.

Department of Chemistry, Institute of Science, Banaras Hindu University, 221005 Varanasi, Uttar Pradesh, India.

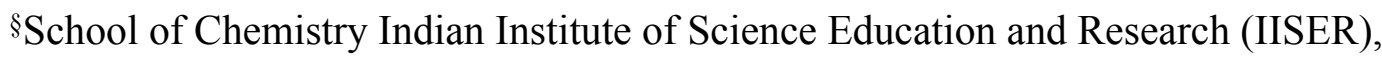
Maruthamala P.O., Vithura, Thiruvananthapuram-695 551, Kerala, India.

$\underline{\text { Table of Contents }}$

\begin{tabular}{|l|l|l|}
\hline 1 & Soft copies of ${ }^{1} \mathrm{H}$ and ${ }^{13} \mathrm{C}\left\{{ }^{1} \mathrm{H}\right\}$ NMR of all compounds & $\mathrm{S} 2-\mathrm{S} 37$ \\
\hline 2 & X-Ray crystal data of 3lb & $\mathrm{S38}-\mathrm{S} 44$ \\
\hline
\end{tabular}


${ }^{1} \mathrm{H}$ NMR $(400 \mathrm{MHz})$ spectra of $\mathbf{2} \mathbf{b}$

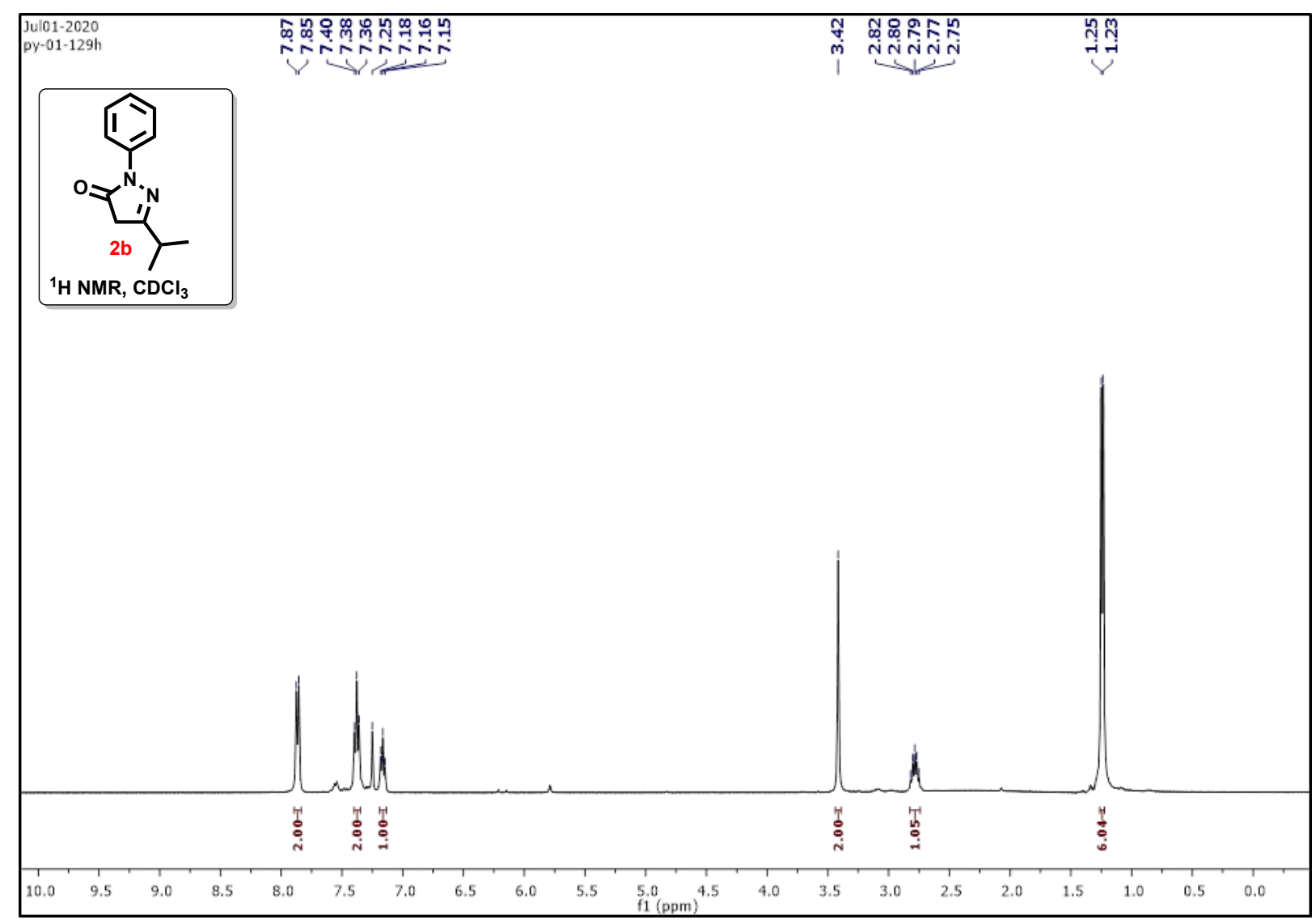

${ }^{13} \mathrm{C}\left\{{ }^{1} \mathrm{H}\right\}$ NMR (101 MHz) spectra of $\mathbf{2} \mathbf{b}$

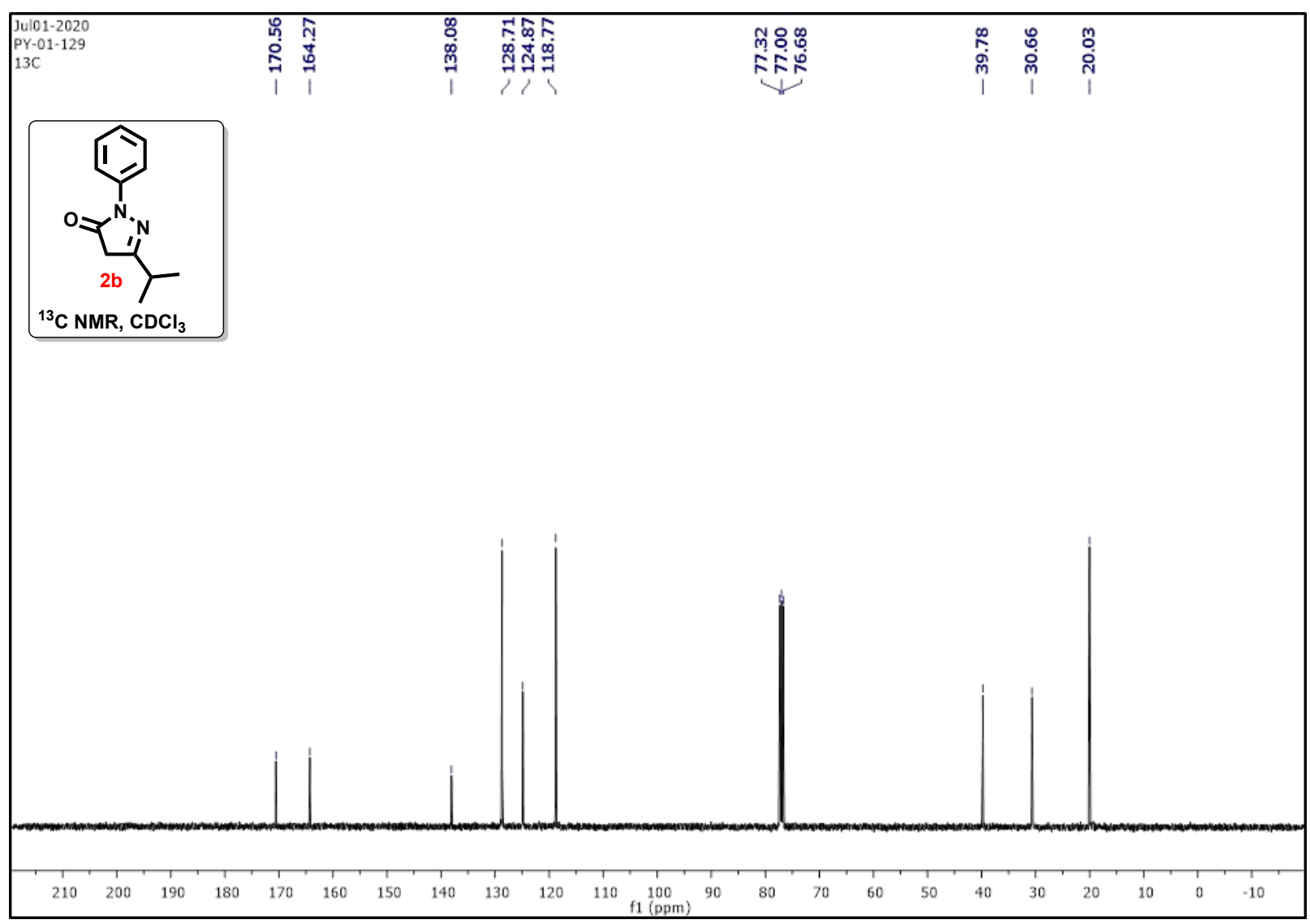


${ }^{1} \mathrm{H}$ NMR $(400 \mathrm{MHz})$ spectra of $\mathbf{2 c}$

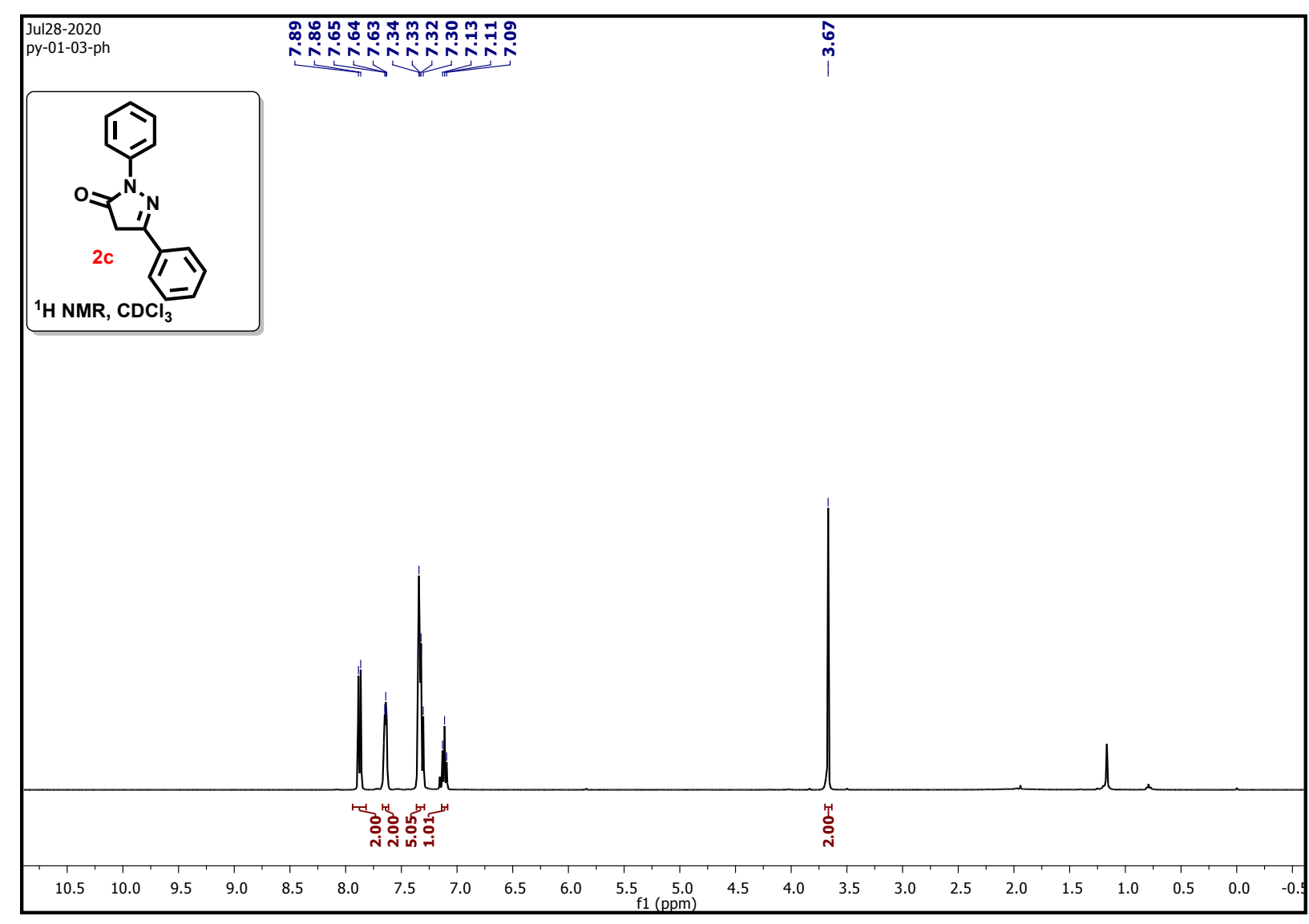

${ }^{13} \mathrm{C}\left\{{ }^{1} \mathrm{H}\right\}$ NMR (101 MHz) spectra of $2 \mathrm{c}$

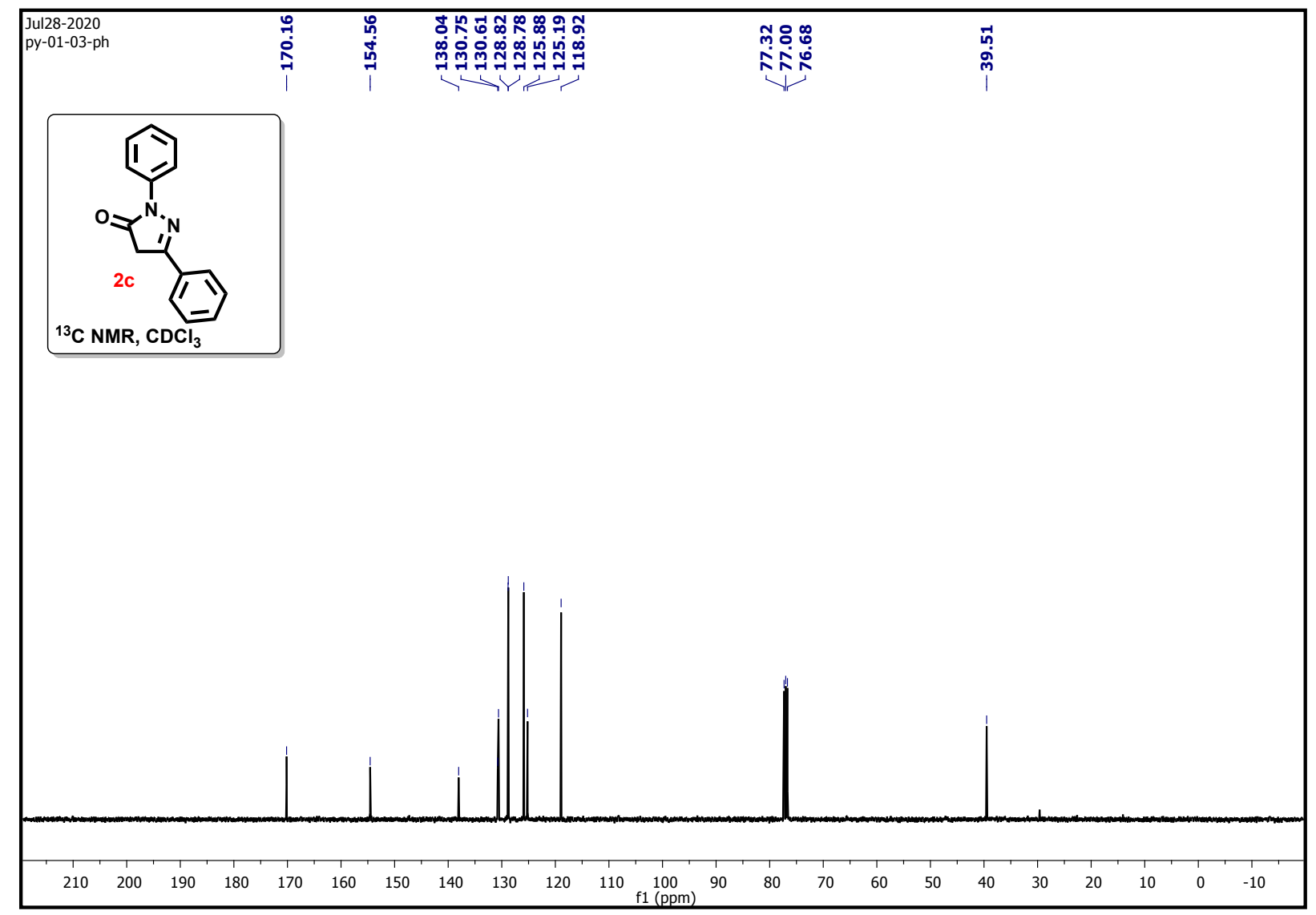


${ }^{1} \mathrm{H}$ NMR $(400 \mathrm{MHz})$ spectra of $\mathbf{2 c}$

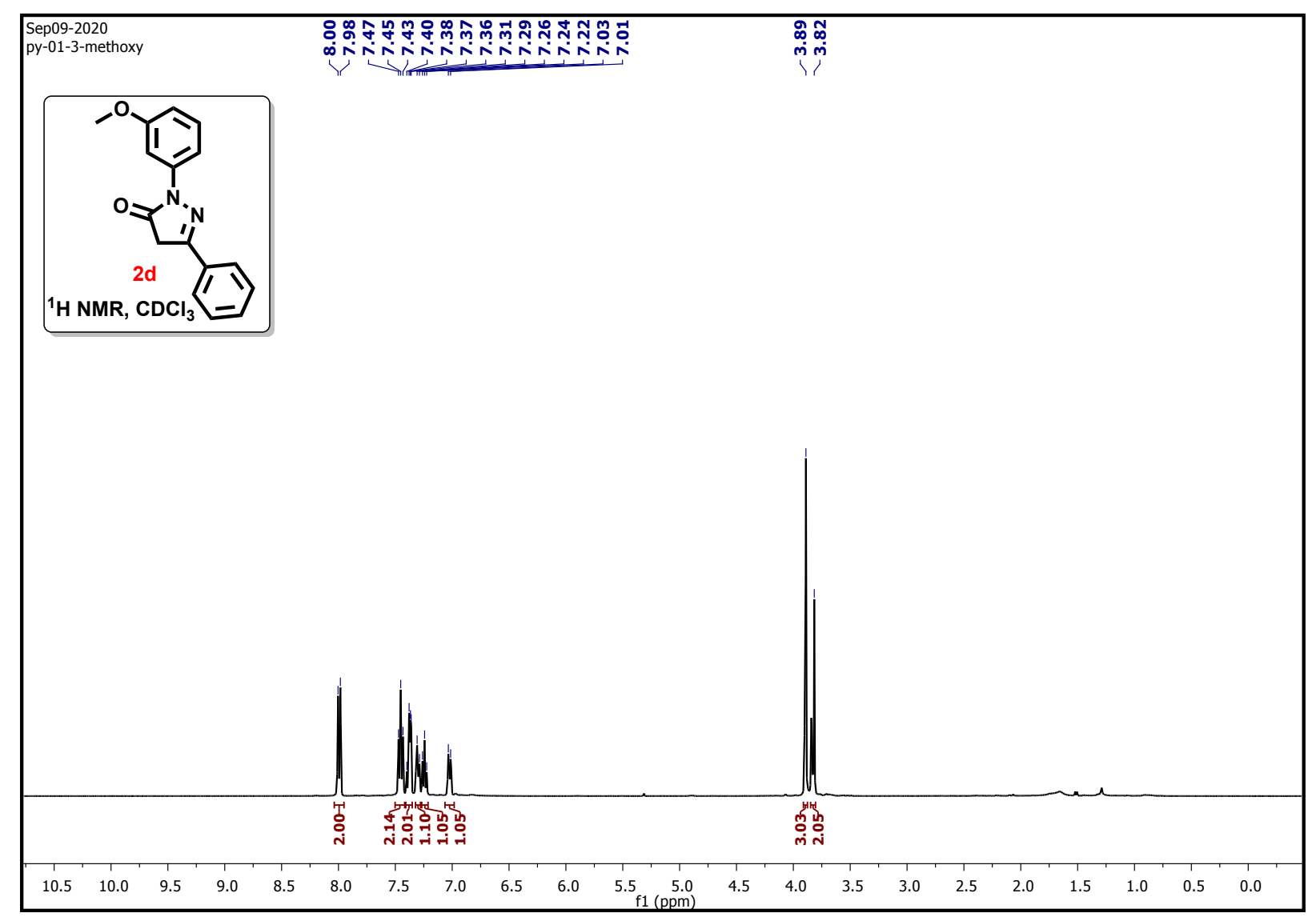

${ }^{13} \mathrm{C}\left\{{ }^{1} \mathrm{H}\right\}$ NMR (101 MHz) spectra of 2c

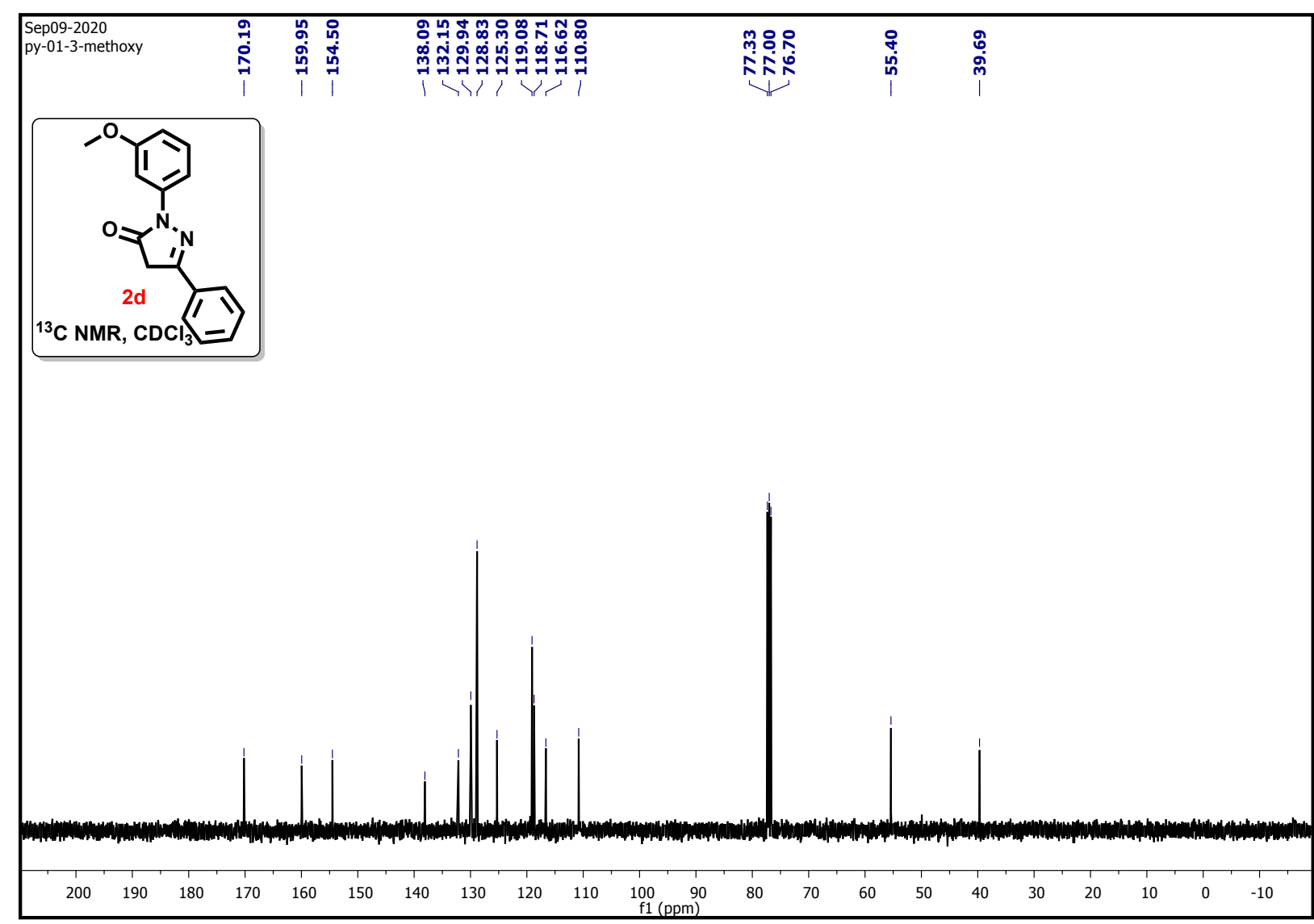


${ }^{1} \mathrm{H}$ NMR (400 MHz) spectra of 3aa

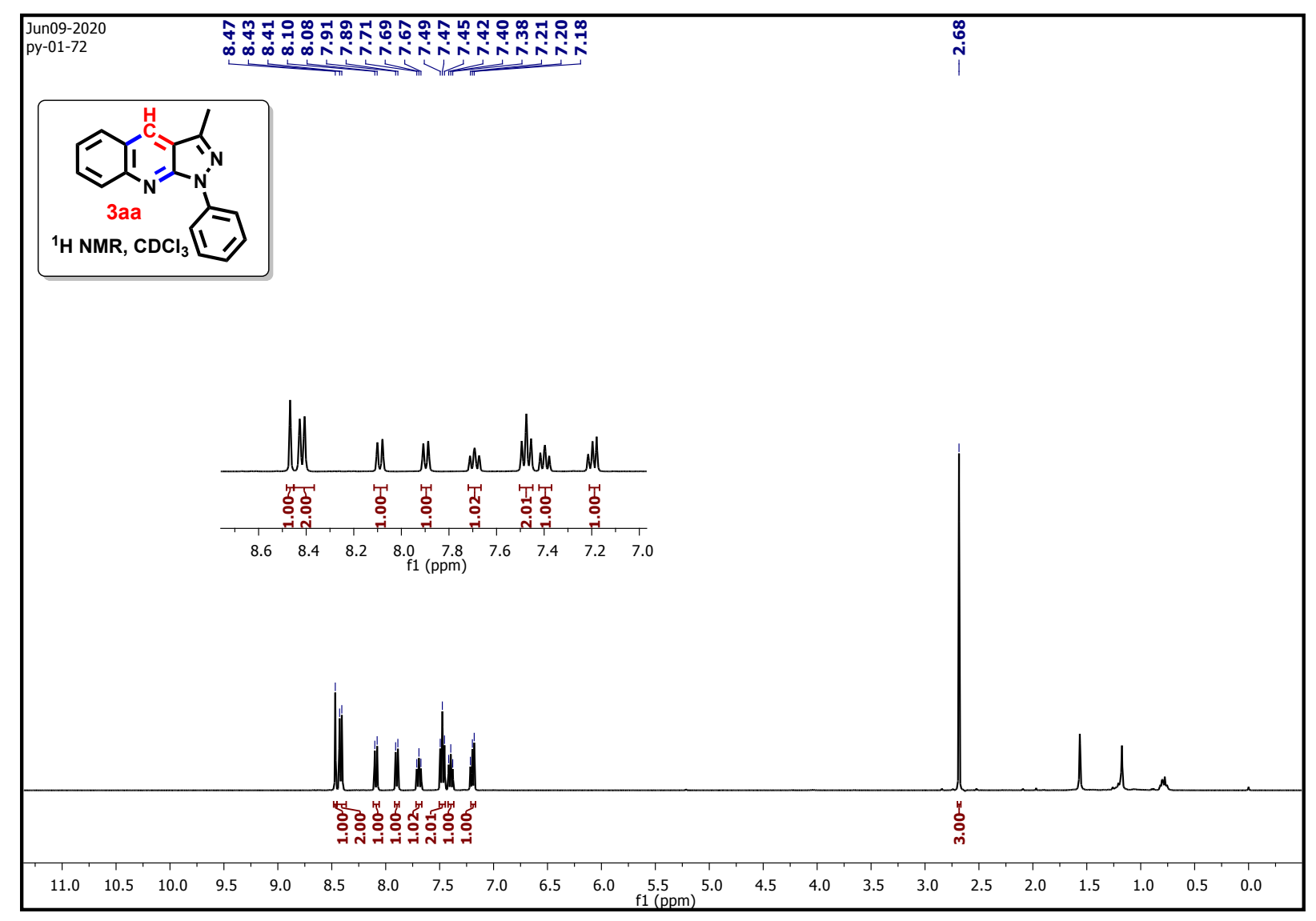

${ }^{13} \mathrm{C}\left\{{ }^{1} \mathrm{H}\right\}$ NMR (101 MHz) spectra of 3aa

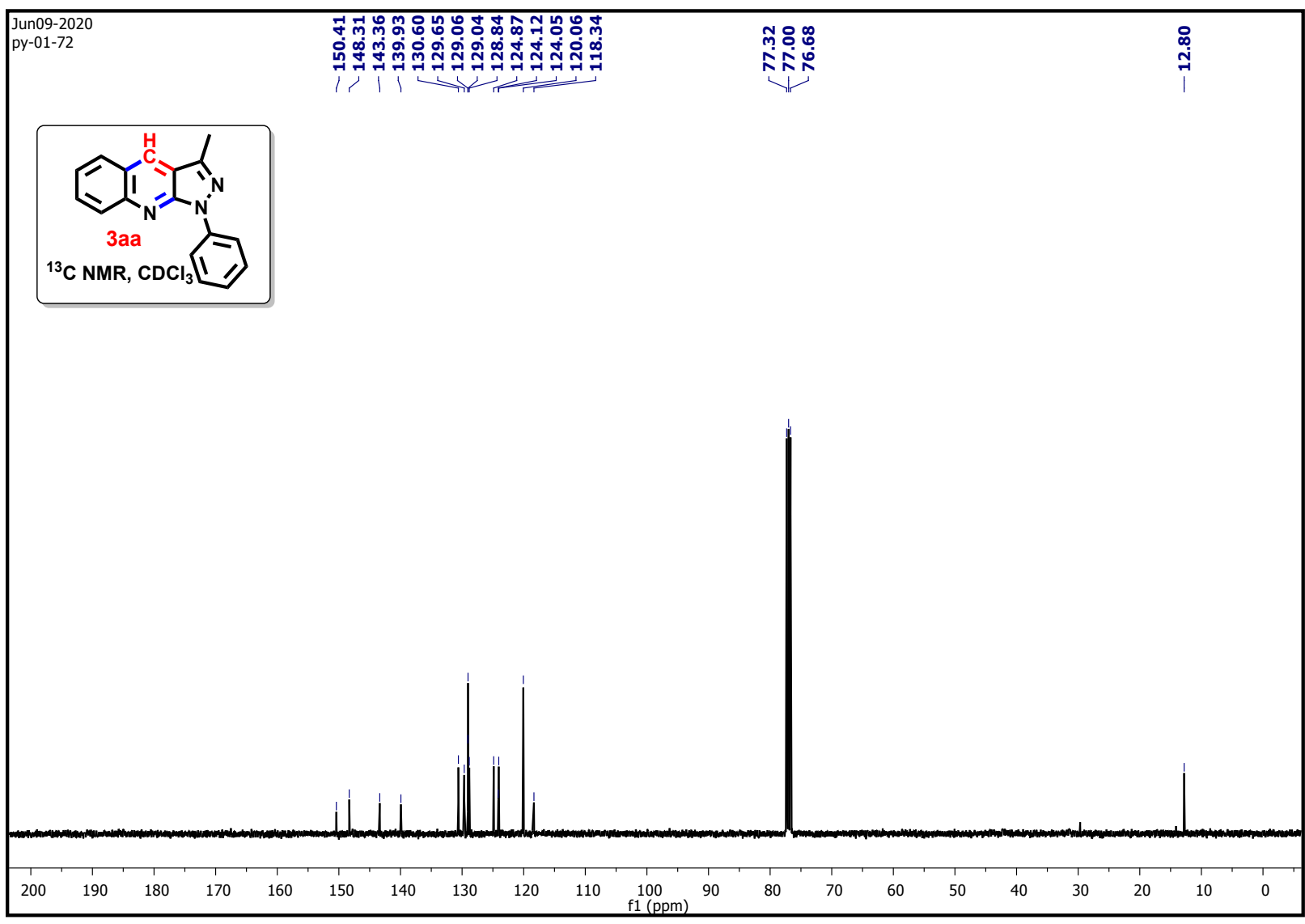


${ }^{1} \mathrm{H}$ NMR $(400 \mathrm{MHz})$ spectra of $\mathbf{3 b a}$

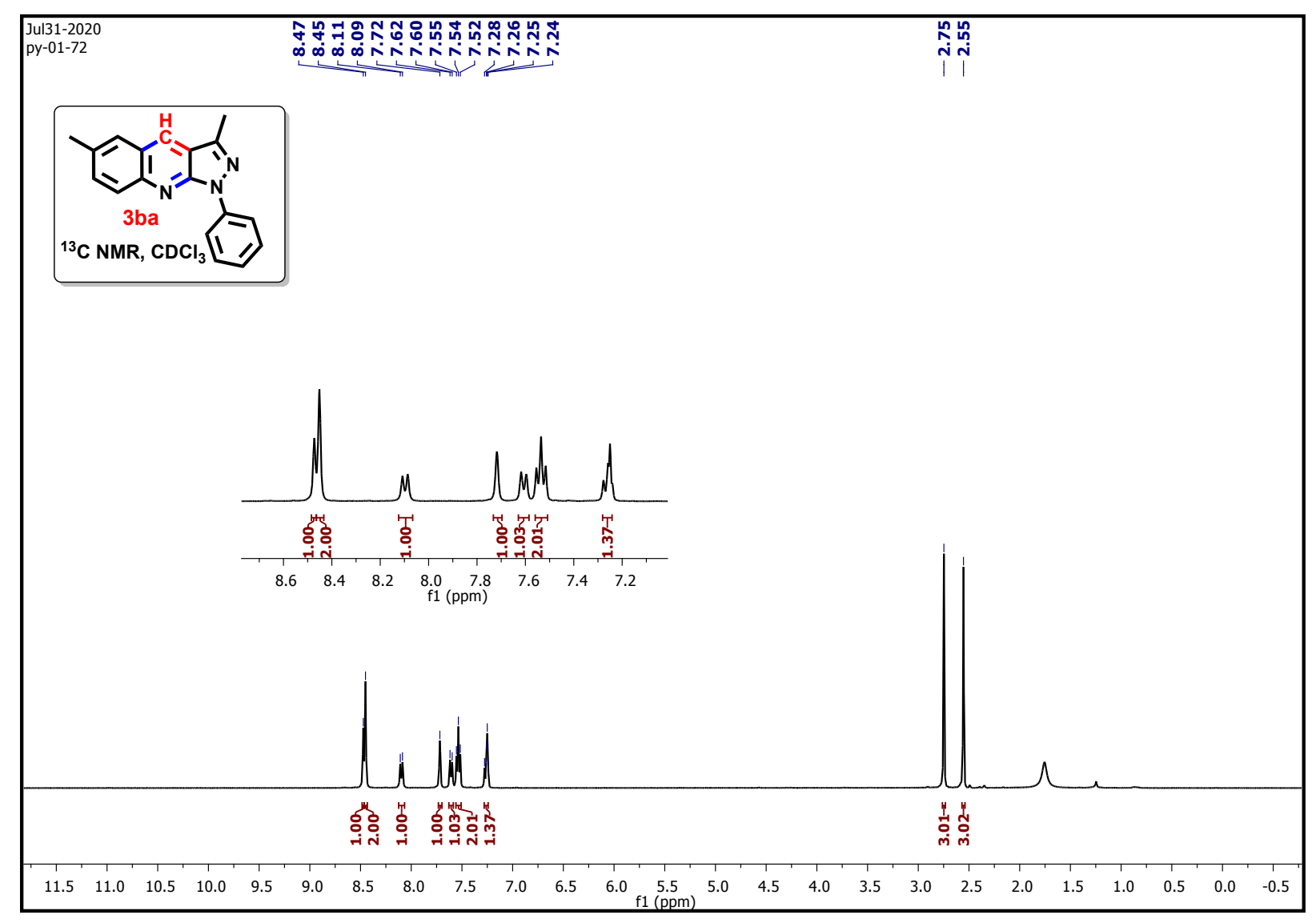

${ }^{13} \mathrm{C}\left\{{ }^{1} \mathrm{H}\right\}$ NMR (101 MHz) spectra of $\mathbf{3 b a}$

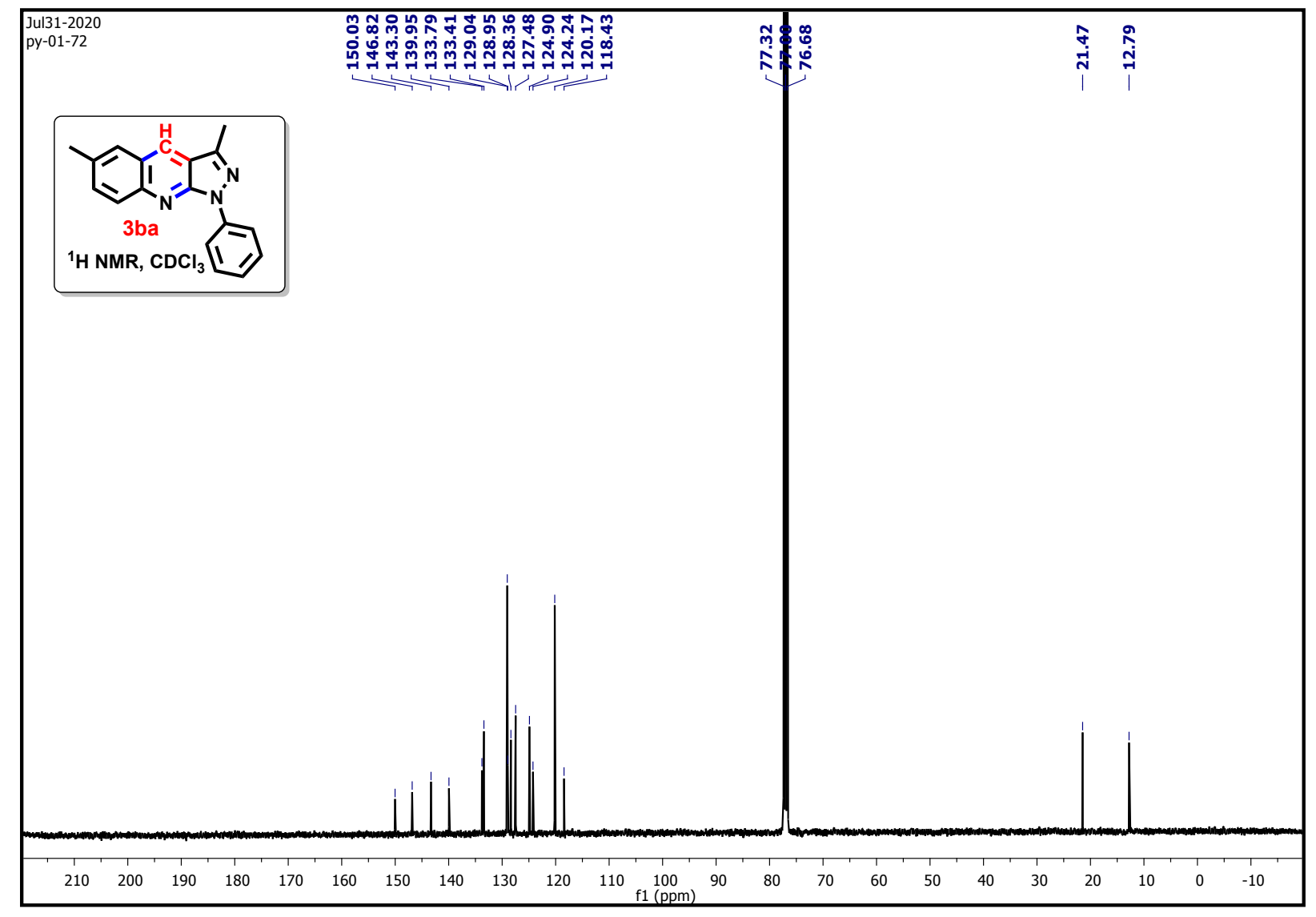


${ }^{1} \mathrm{H}$ NMR (400 MHz) spectra of $3 \mathrm{ca}$

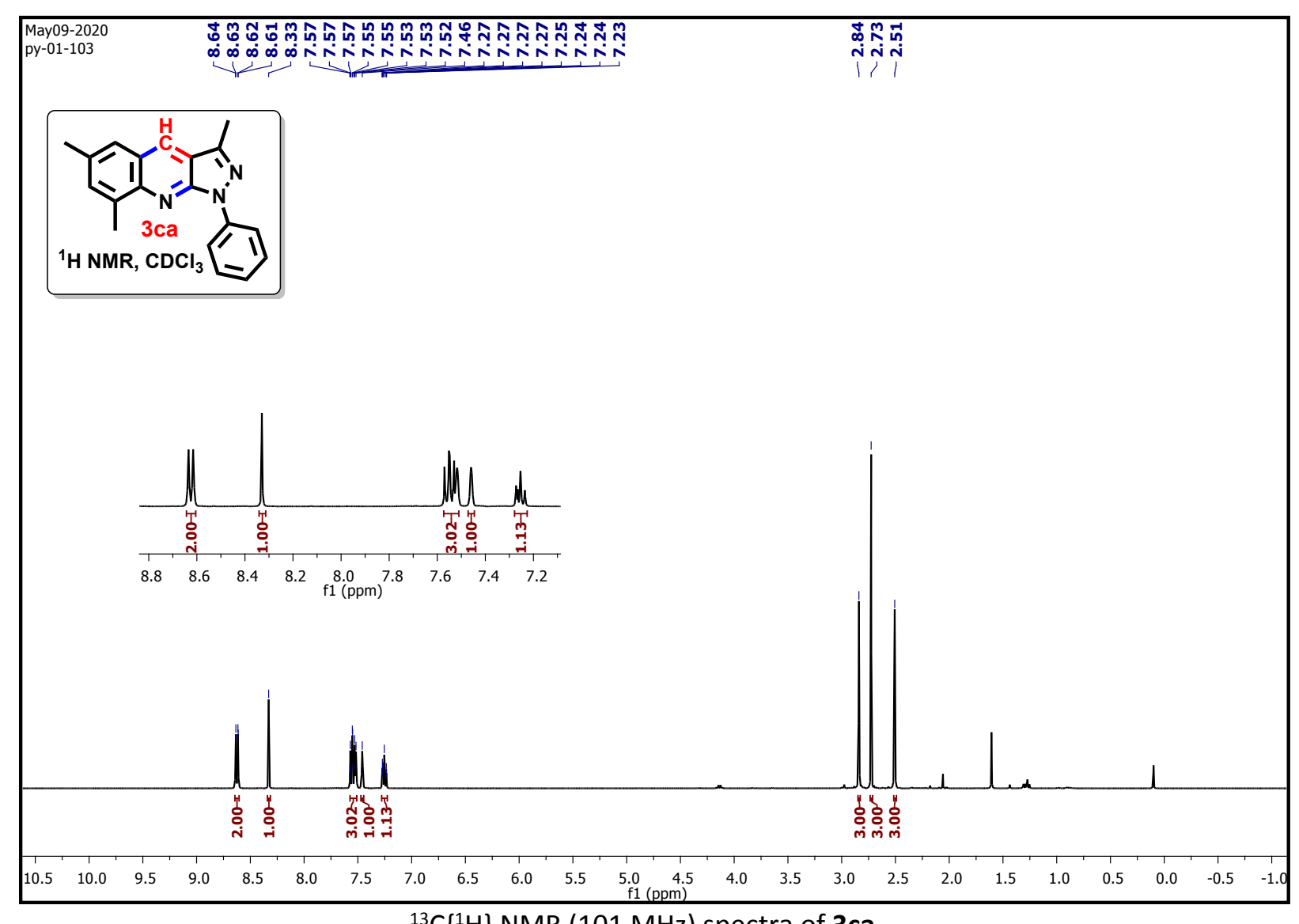

${ }^{13} \mathrm{C}\left\{{ }^{1} \mathrm{H}\right\}$ NMR (101 MHz) spectra of 3ca

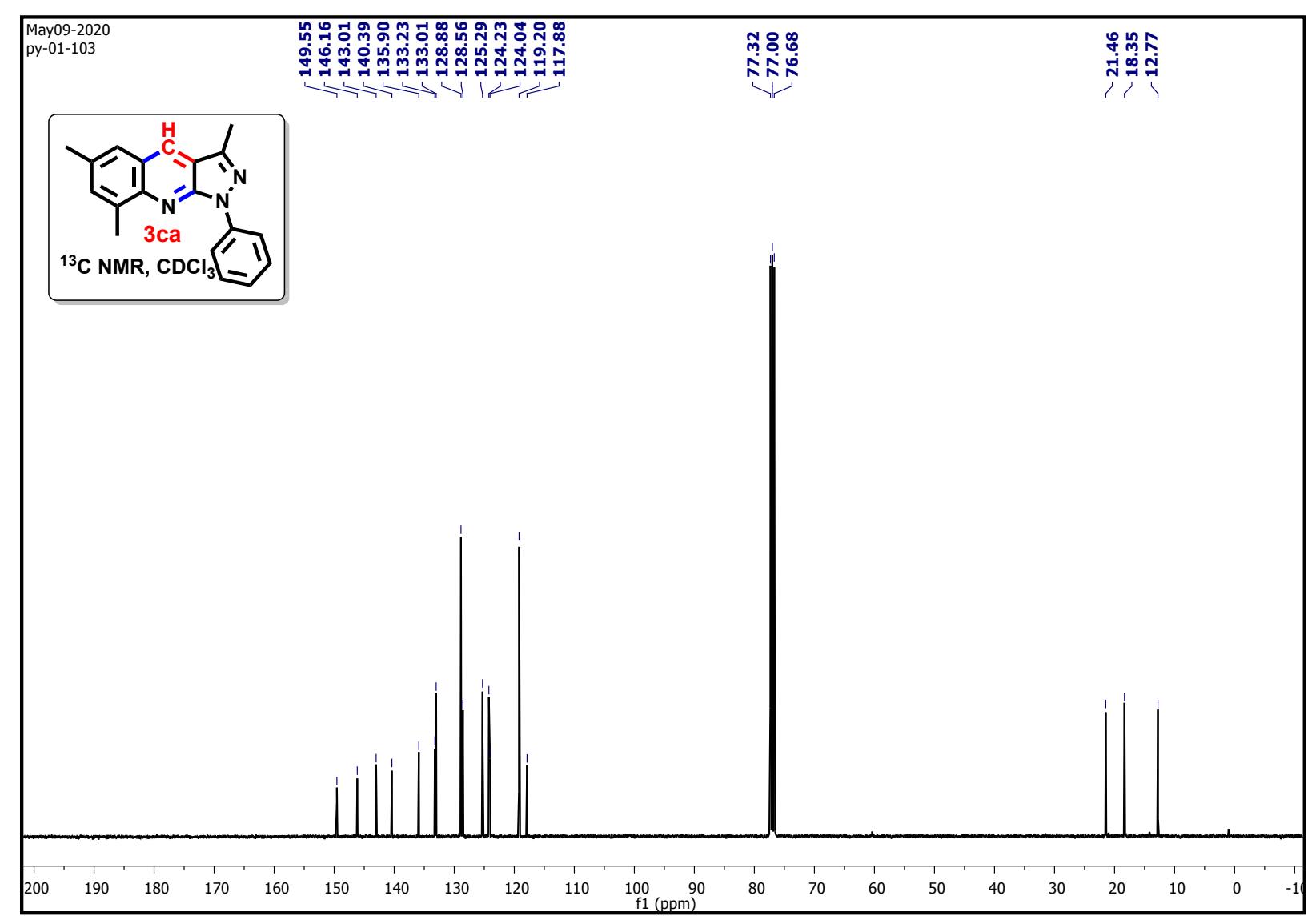


${ }^{1} \mathrm{H}$ NMR (400 MHz) spectra of $\mathbf{3 d a}$

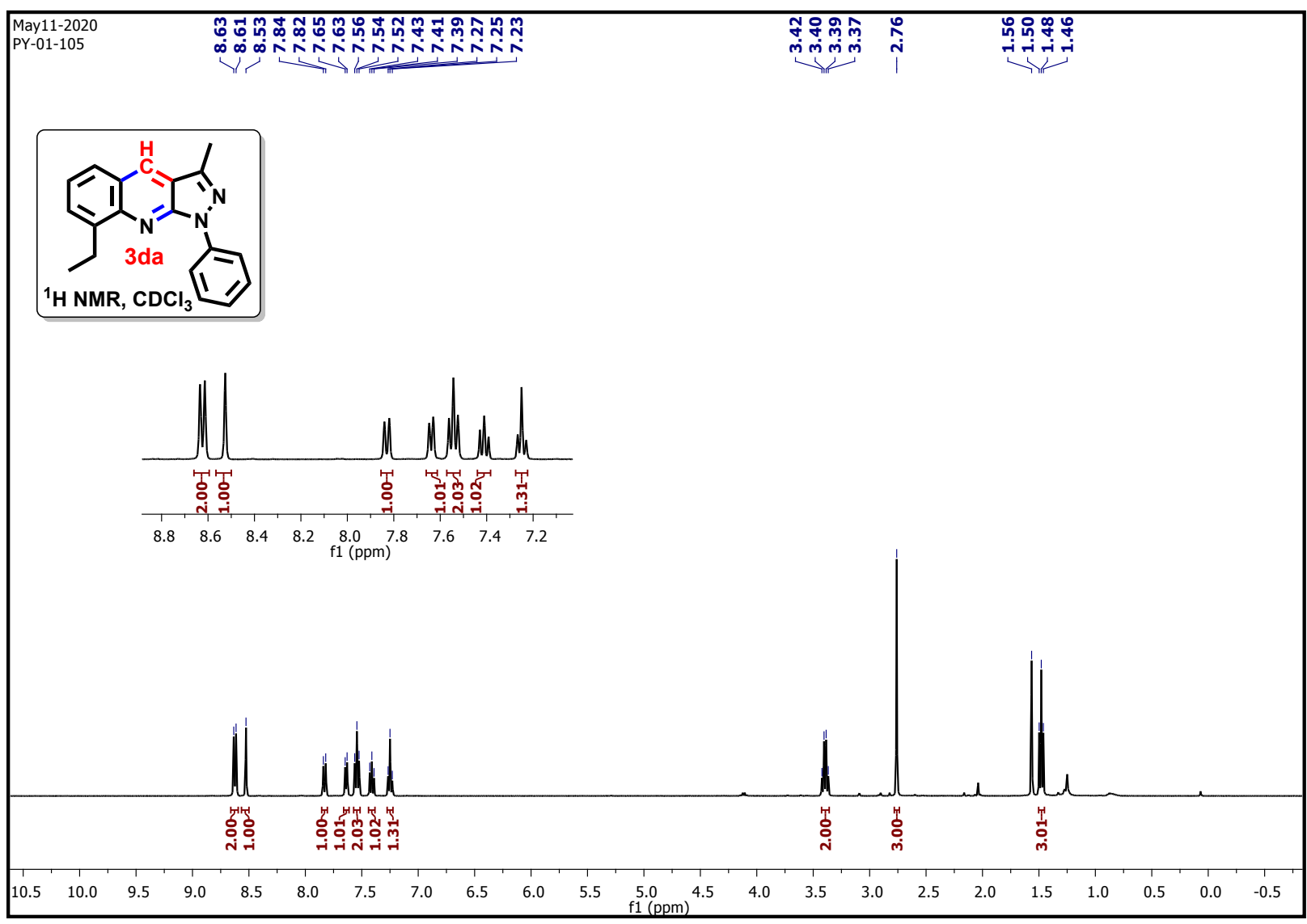

${ }^{13} \mathrm{C}\left\{{ }^{1} \mathrm{H}\right\}$ NMR (101 MHz) spectra of $\mathbf{3 c a}$

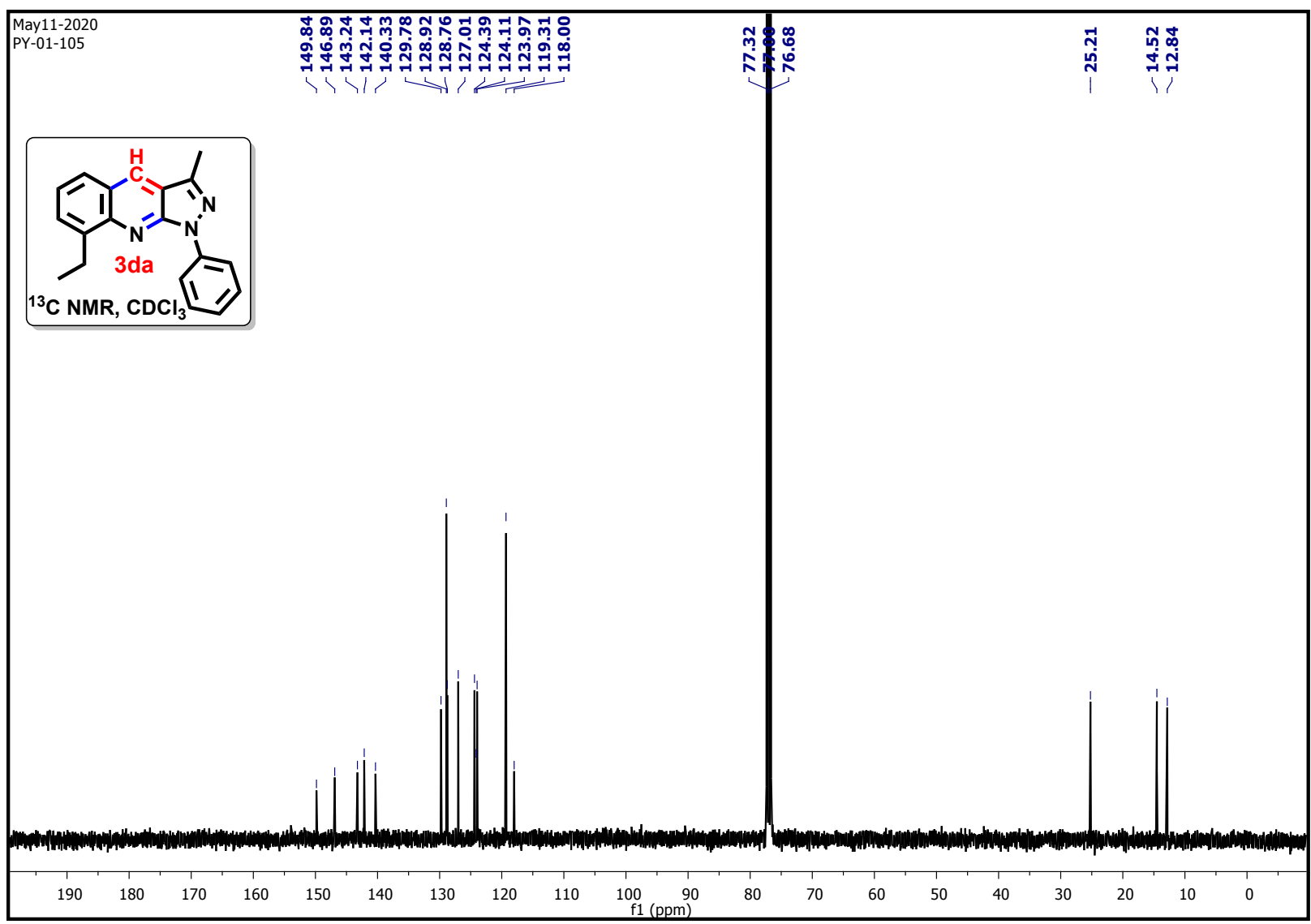


${ }^{1} \mathrm{H}$ NMR (400 MHz) spectra of 3ea

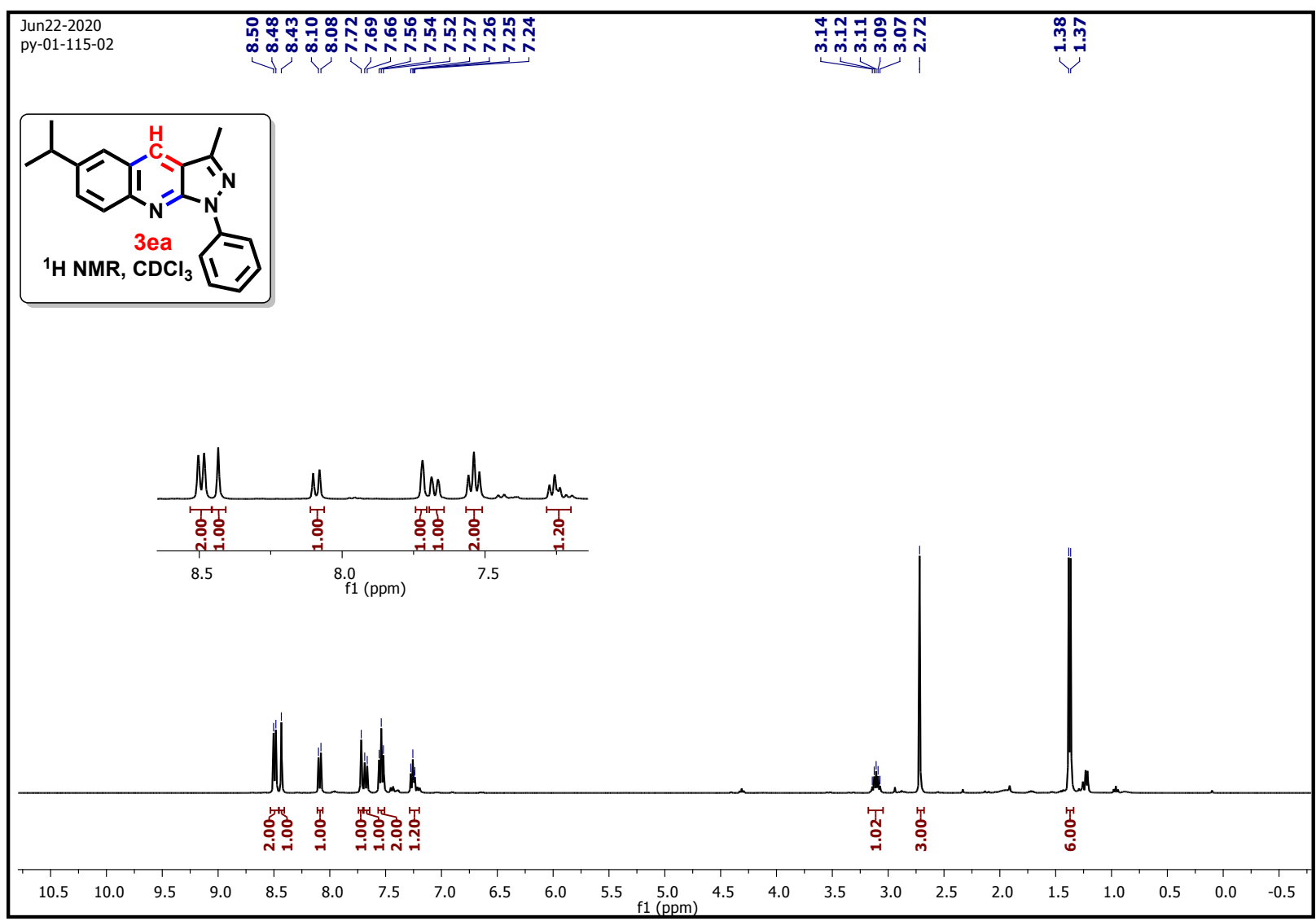

${ }^{13} \mathrm{C}\left\{{ }^{1} \mathrm{H}\right\}$ NMR (101 MHz) spectra of 3ea

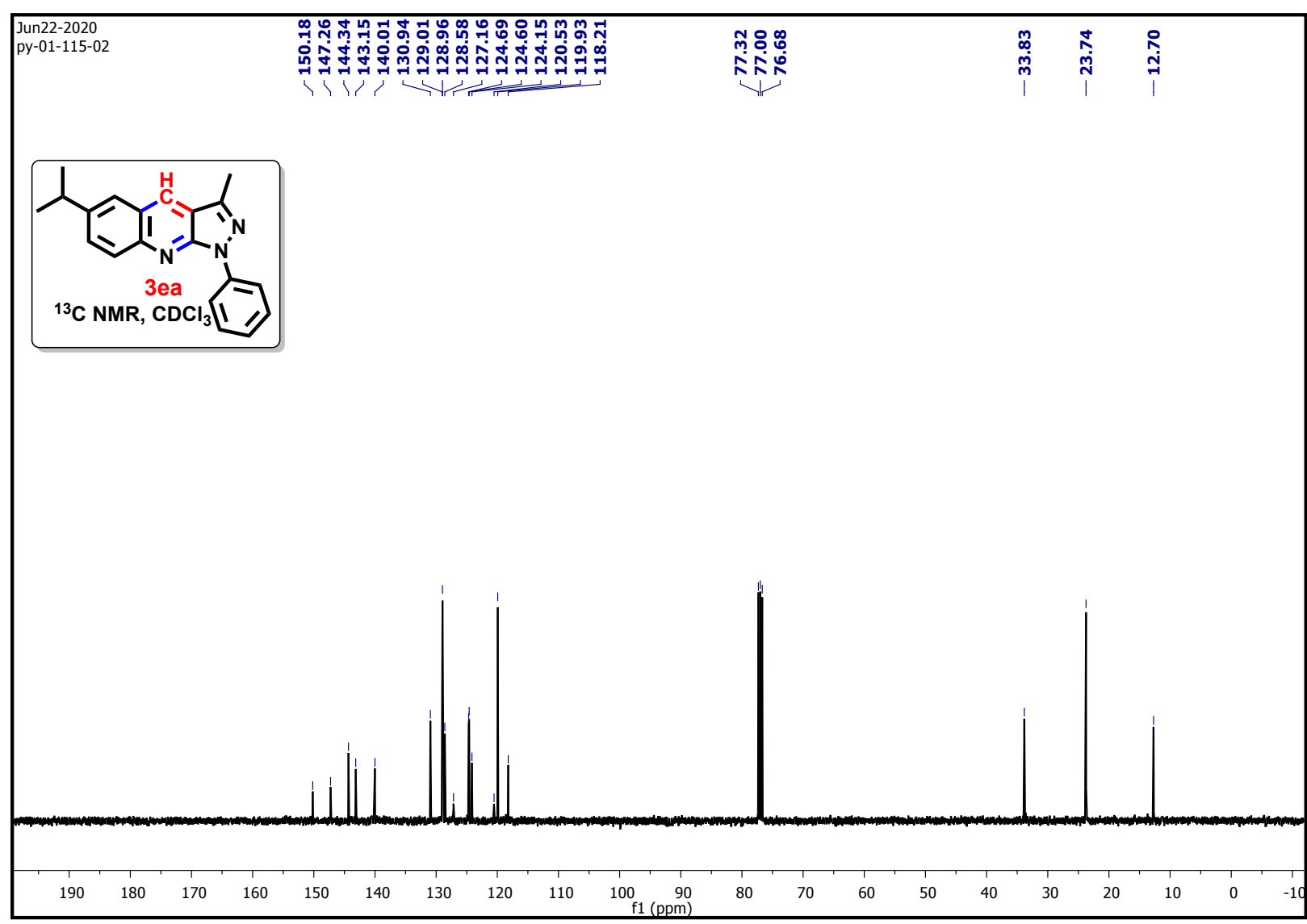


${ }^{1} \mathrm{H}$ NMR (400 MHz) spectra of $\mathbf{3 f a}$

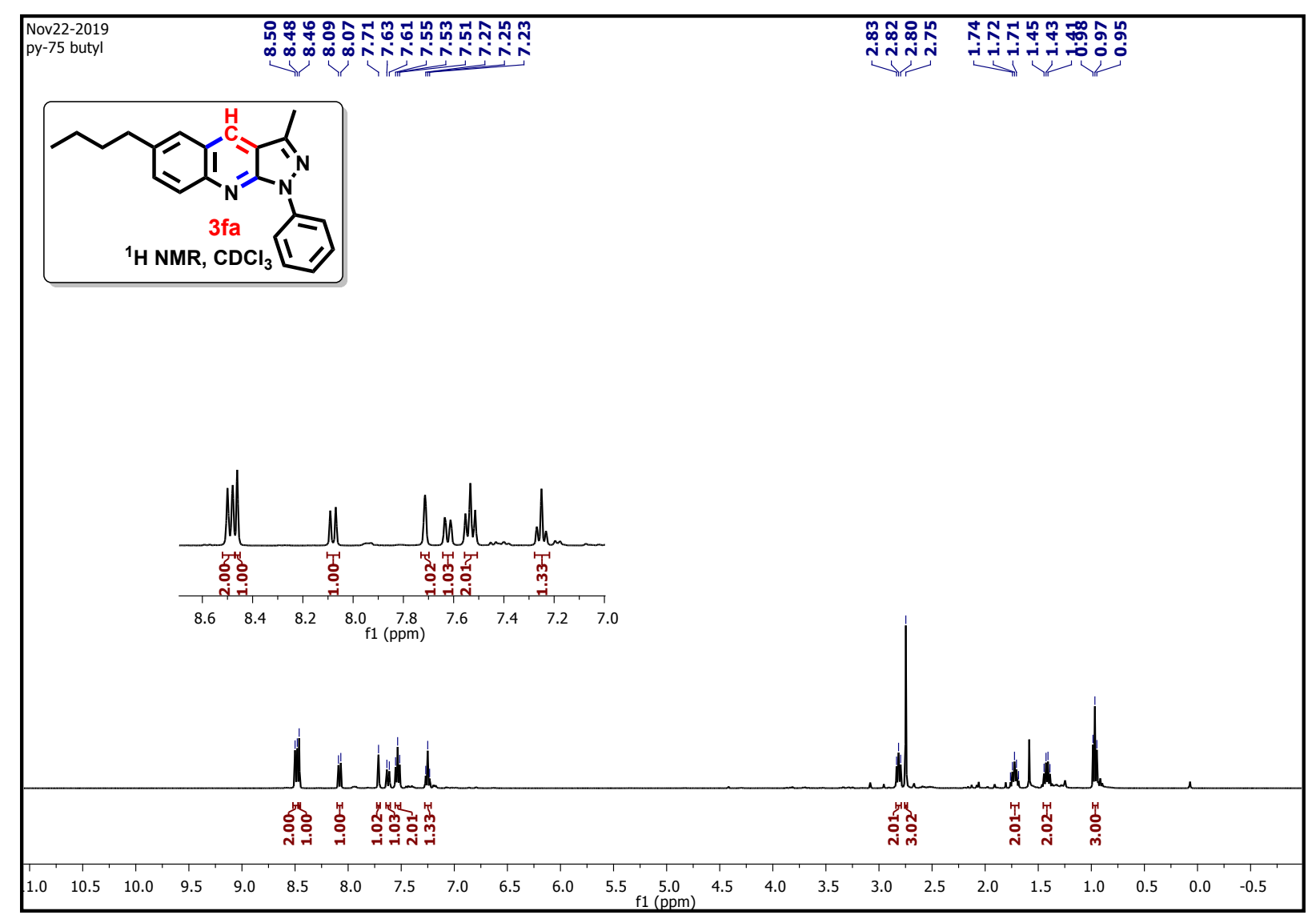

${ }^{13} \mathrm{C}\left\{{ }^{1} \mathrm{H}\right\}$ NMR $(101 \mathrm{MHz})$ spectra of $\mathbf{3 f a}$

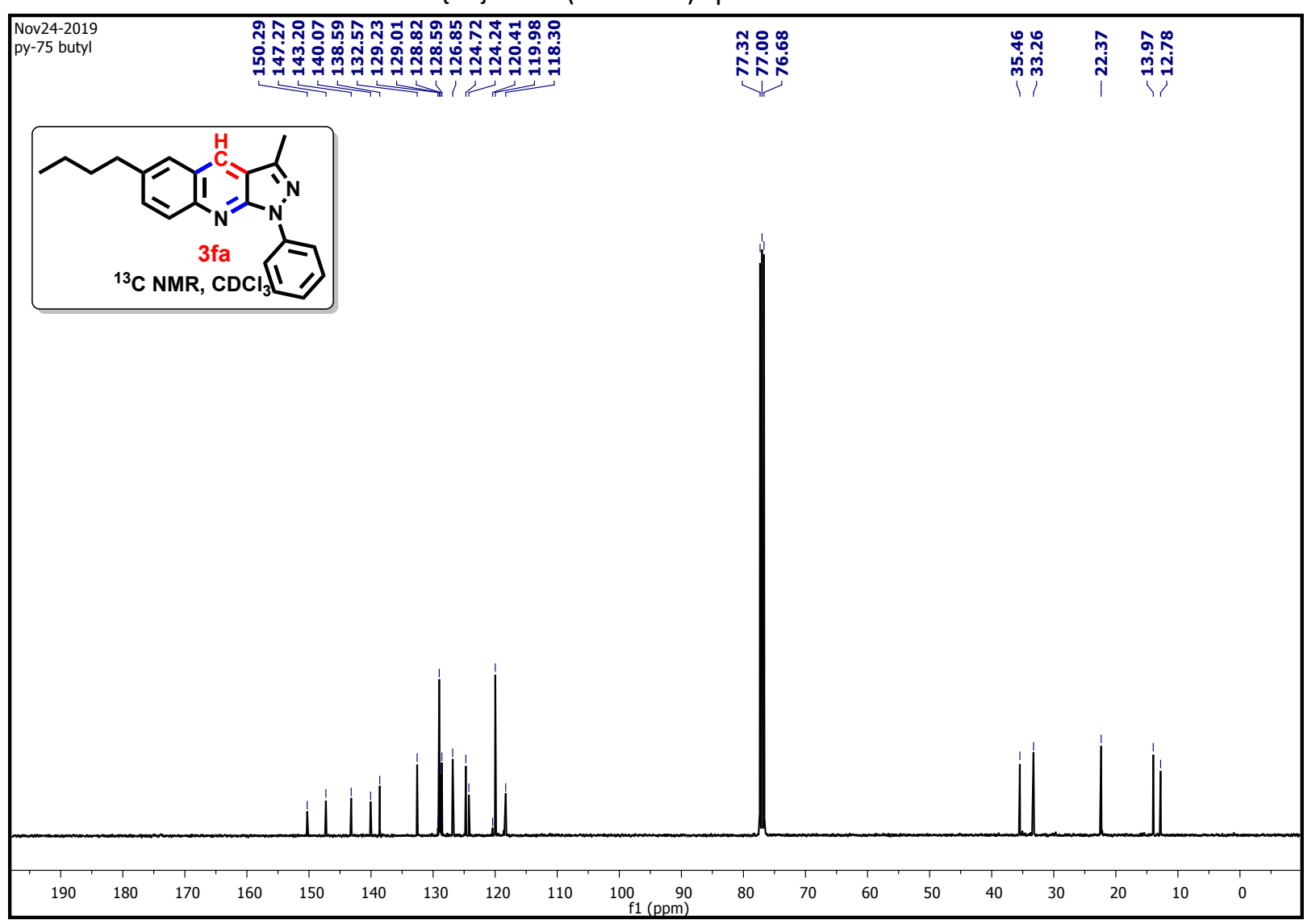


${ }^{1} \mathrm{H}$ NMR (400 MHz) spectra of 3ga

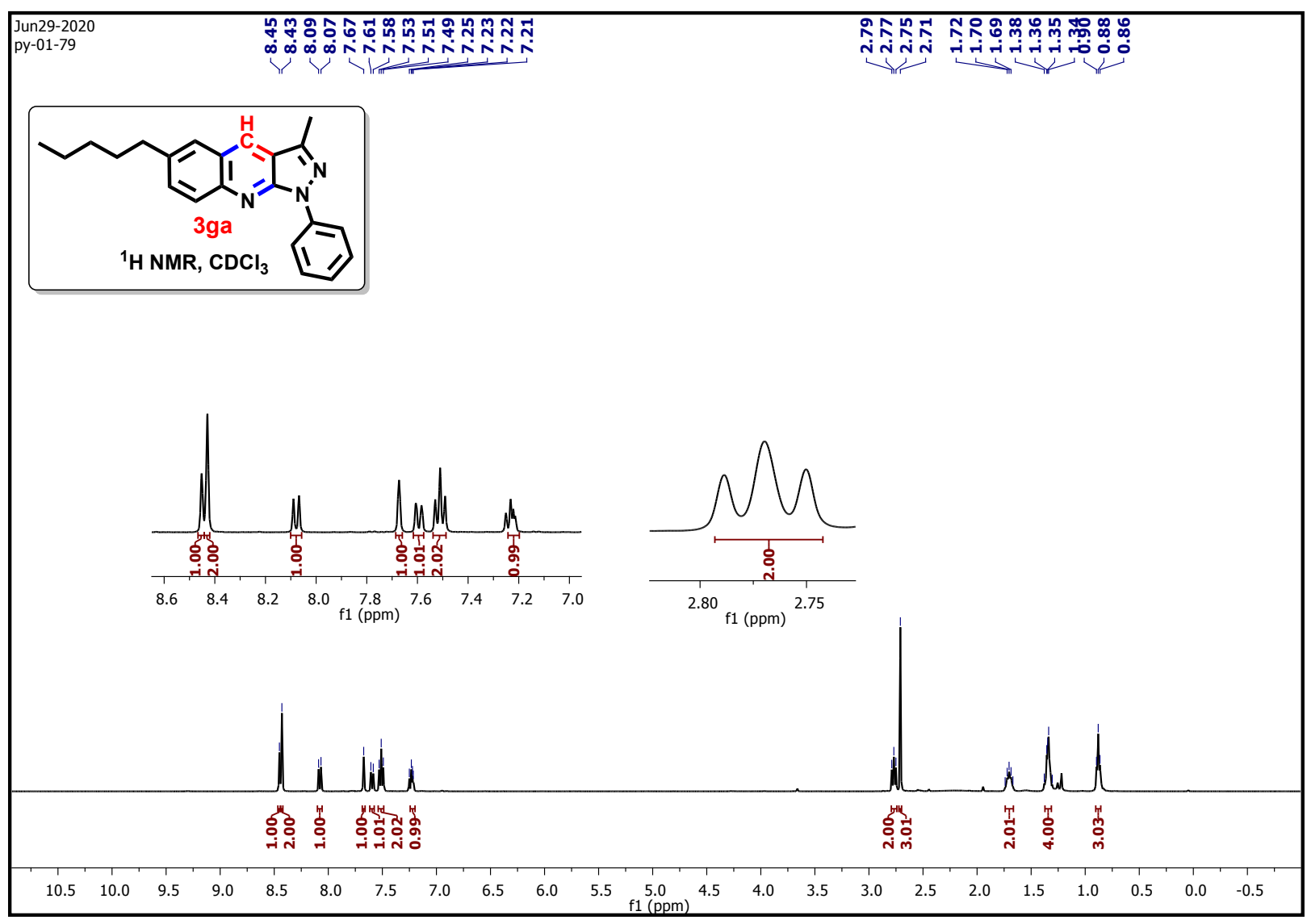

${ }^{13} \mathrm{C}\left\{{ }^{1} \mathrm{H}\right\}$ NMR (101 MHz) spectra of 3ga

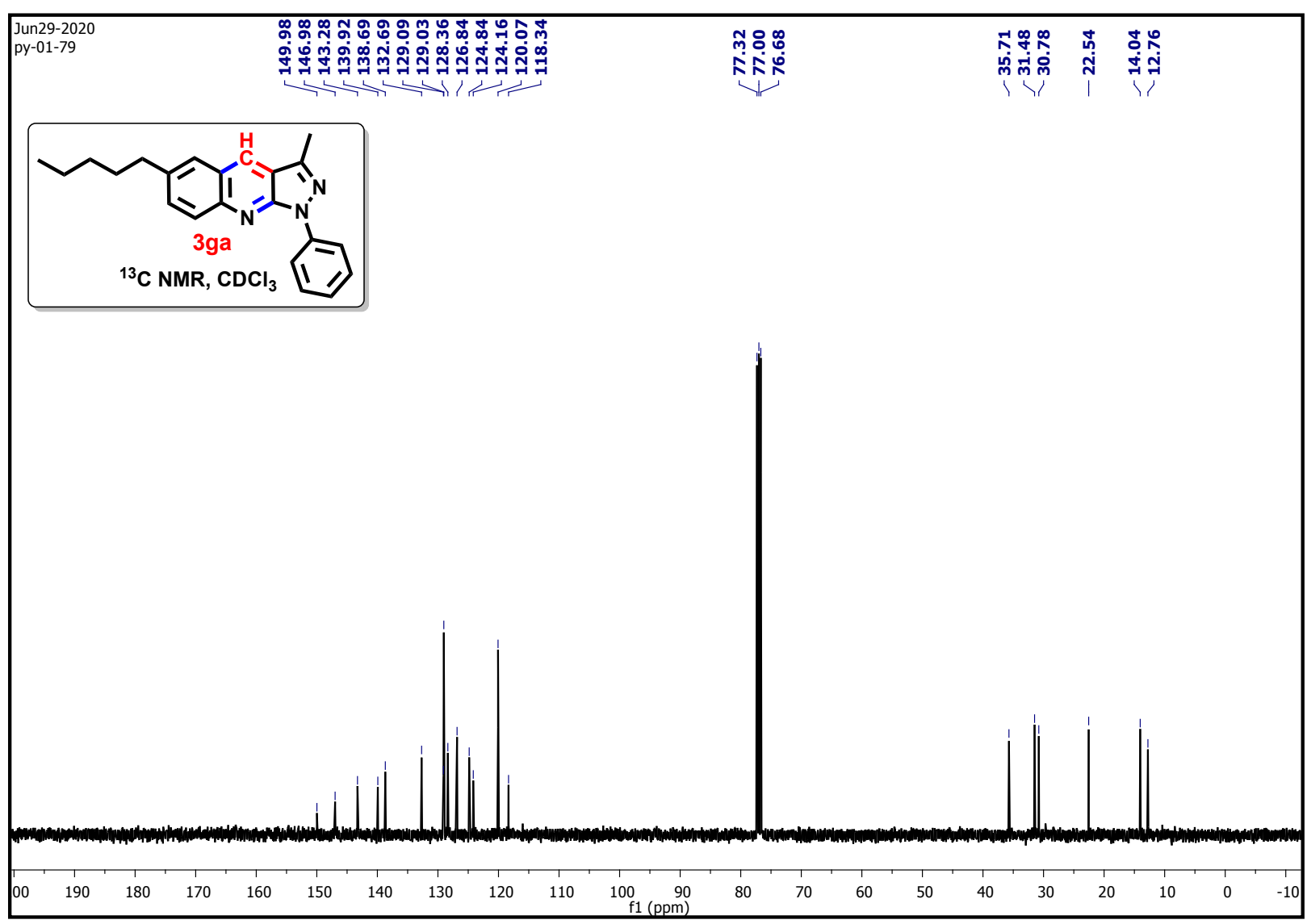


${ }^{1} \mathrm{H}$ NMR (400 MHz) spectra of 3ha

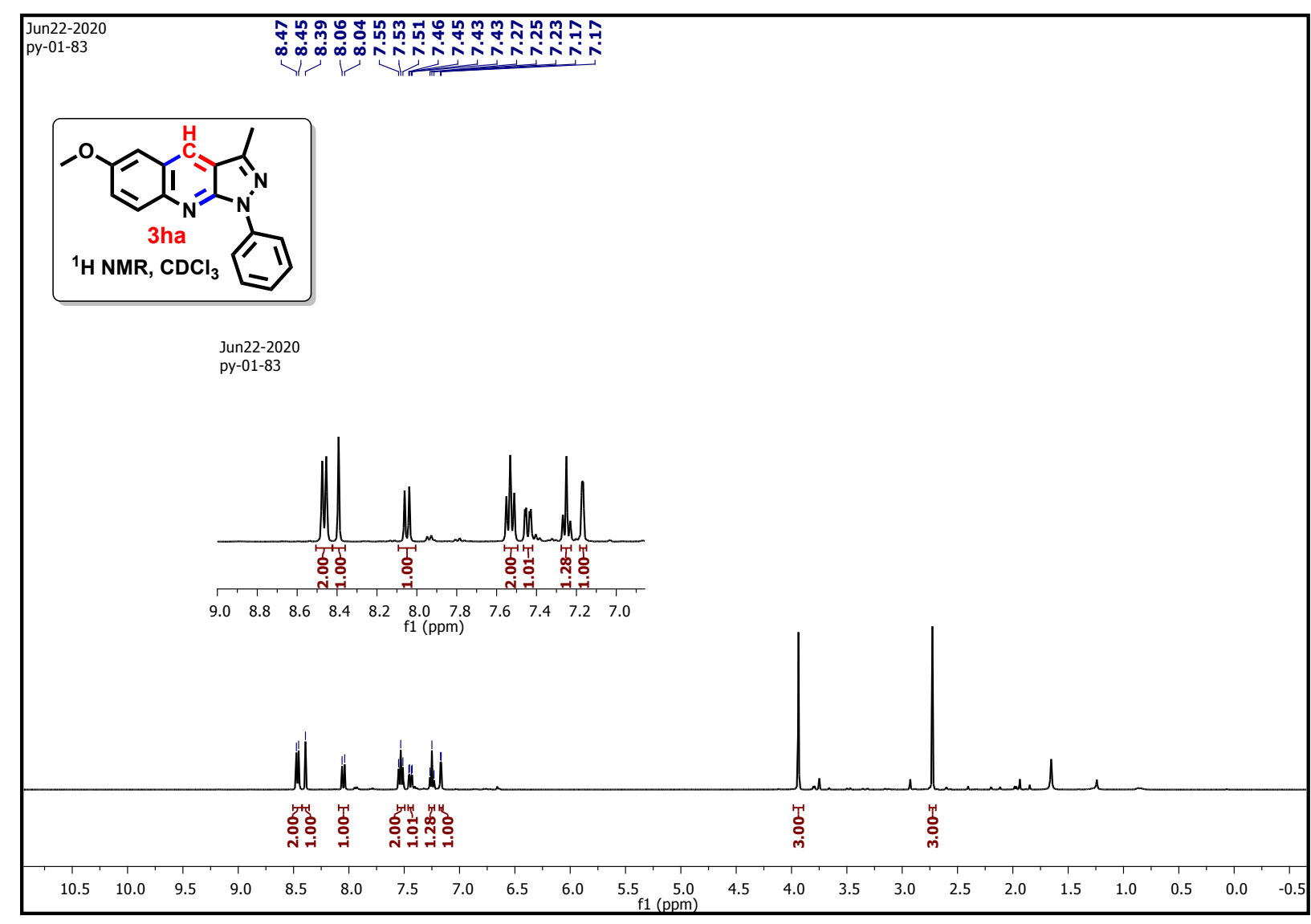

${ }^{13} \mathrm{C}\left\{{ }^{1} \mathrm{H}\right\}$ NMR (101 MHz) spectra of 3 ha

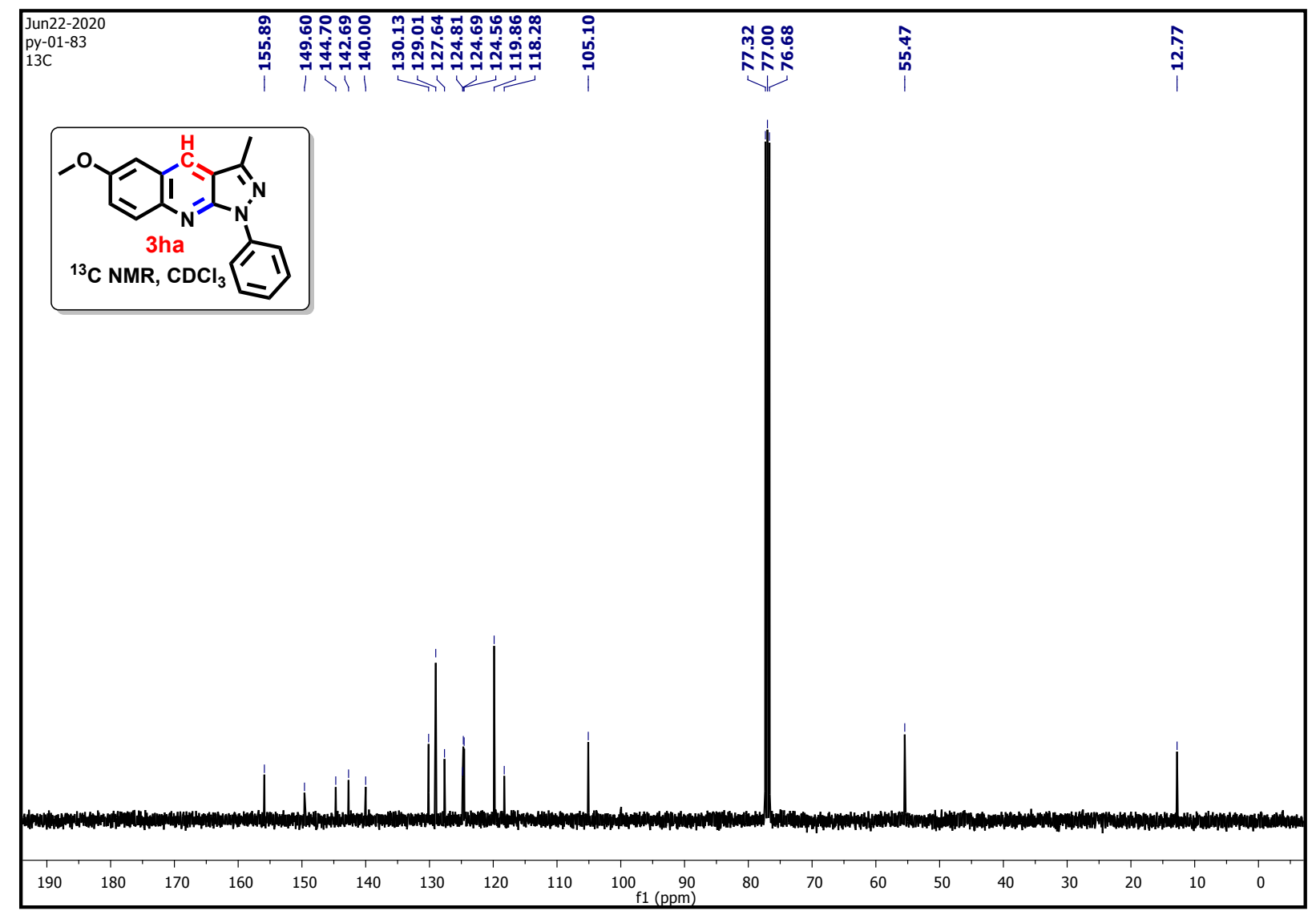


${ }^{1} \mathrm{H}$ NMR (400 MHz) spectra of 3ia

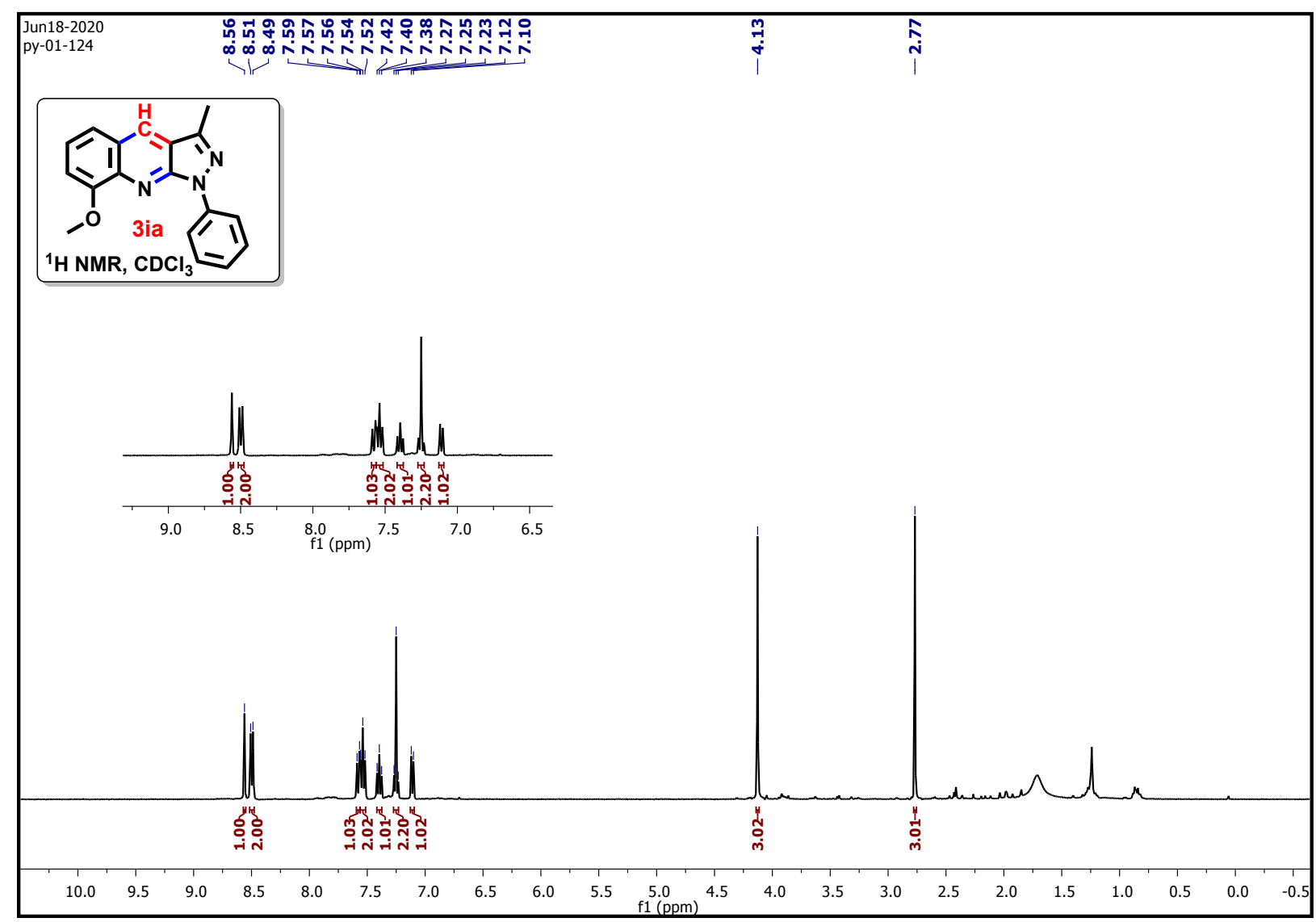

${ }^{13} \mathrm{C}\left\{{ }^{1} \mathrm{H}\right\}$ NMR (101 MHz) spectra of 3ia

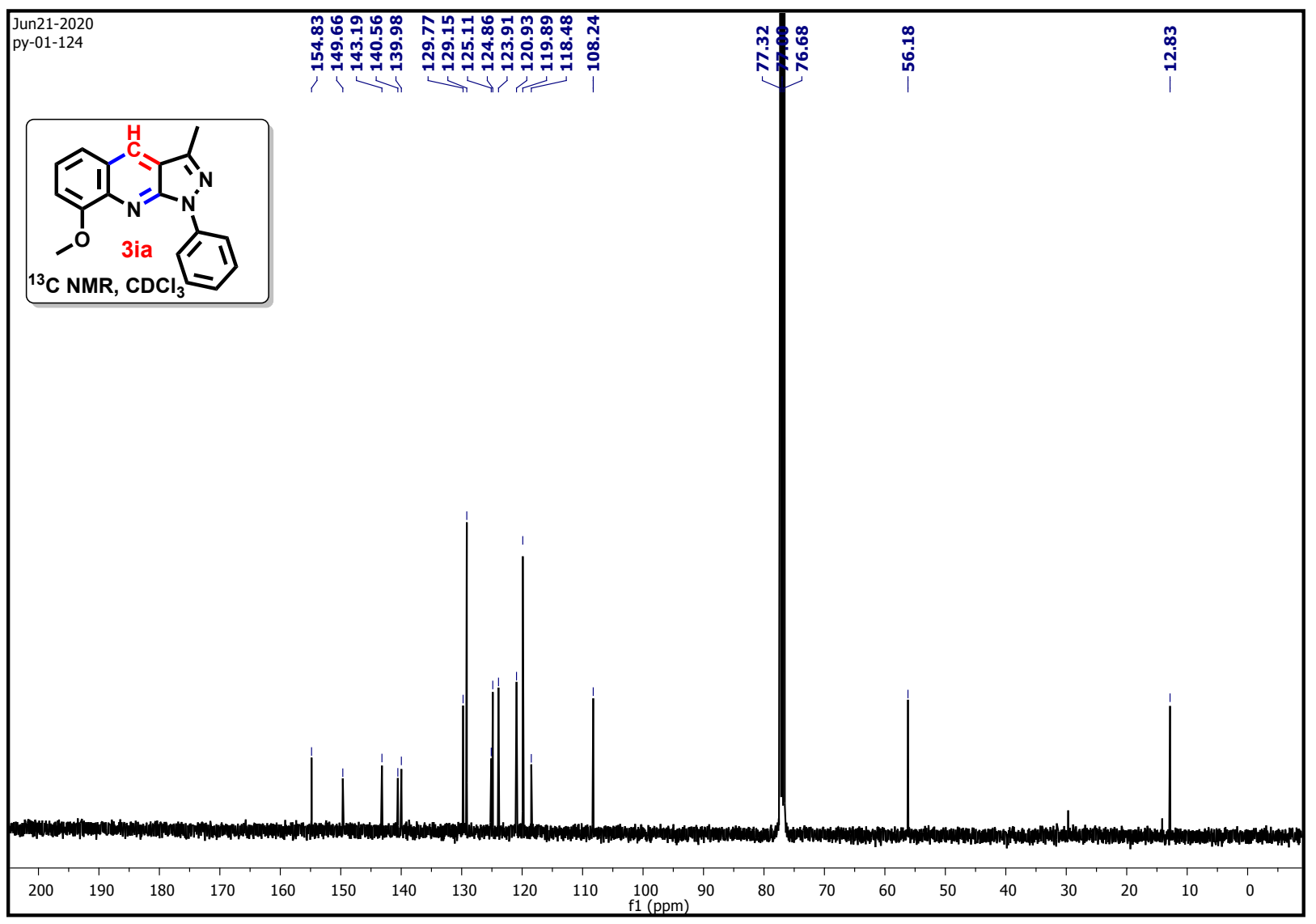


${ }^{1} \mathrm{H}$ NMR (400 MHz) spectra of 3ja

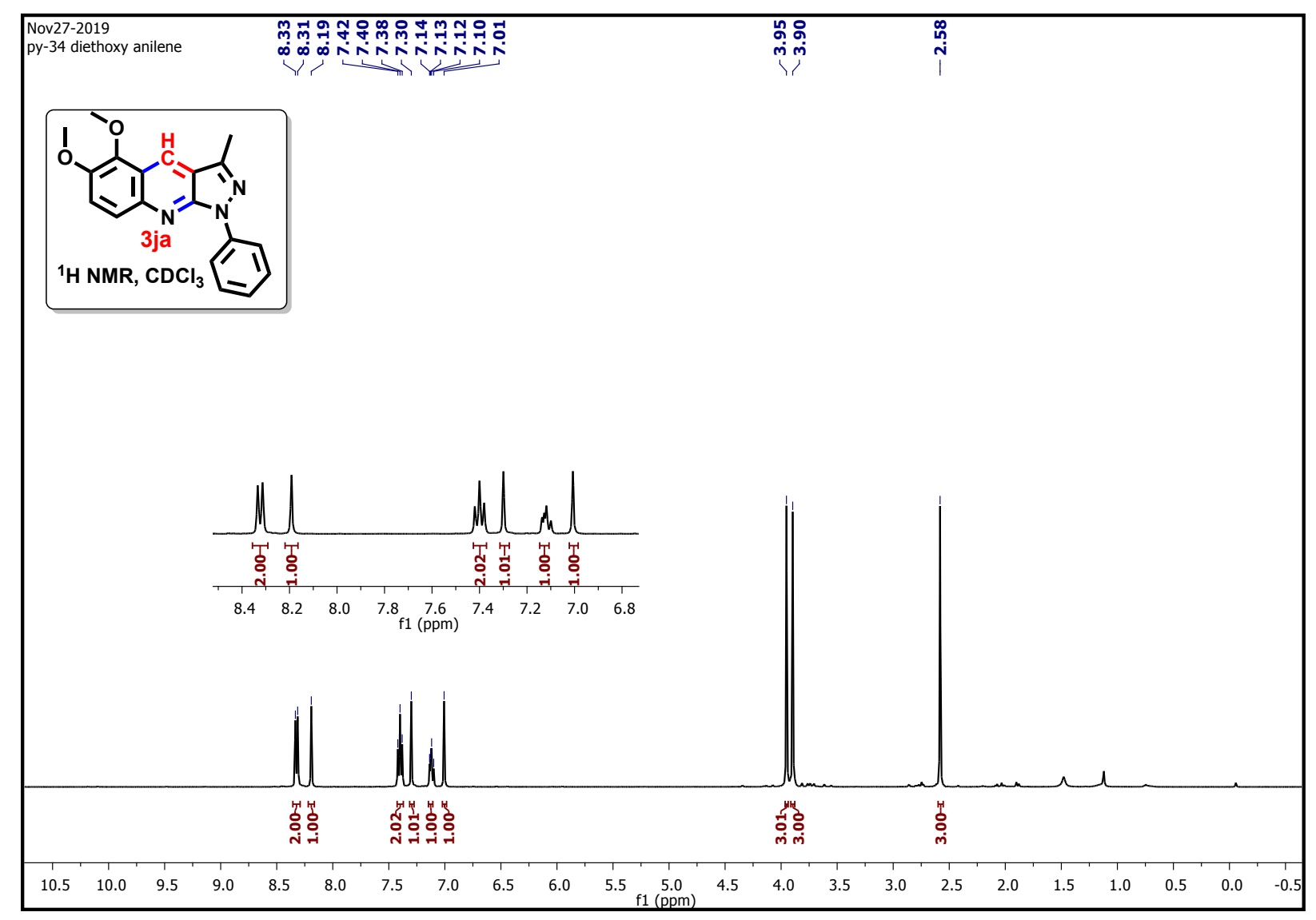

${ }^{13} \mathrm{C}\left\{{ }^{1} \mathrm{H}\right\}$ NMR (101 MHz) spectra of 3ja

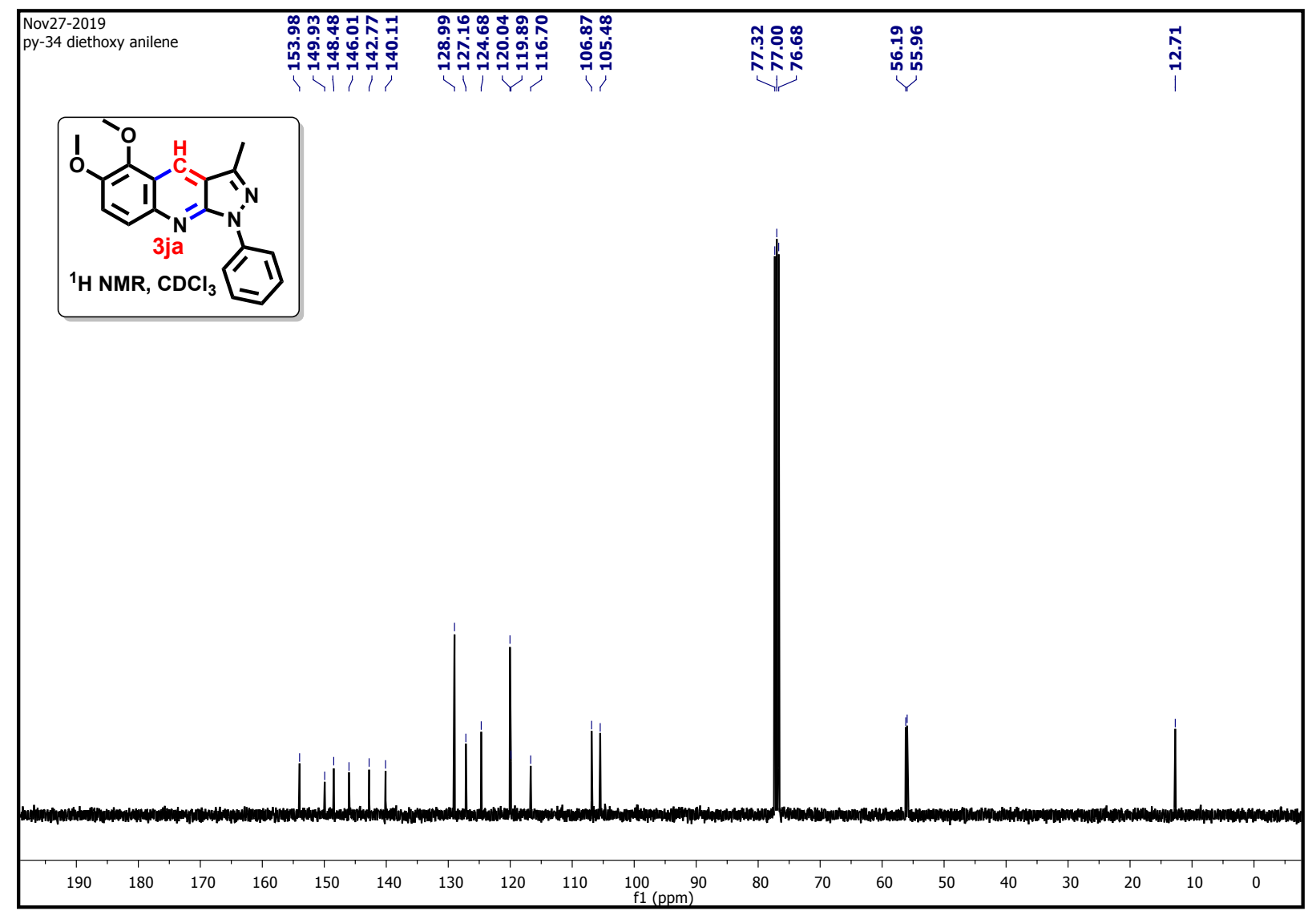


${ }^{1} \mathrm{H}$ NMR (800 MHz) spectra of $\mathbf{3 k a}$

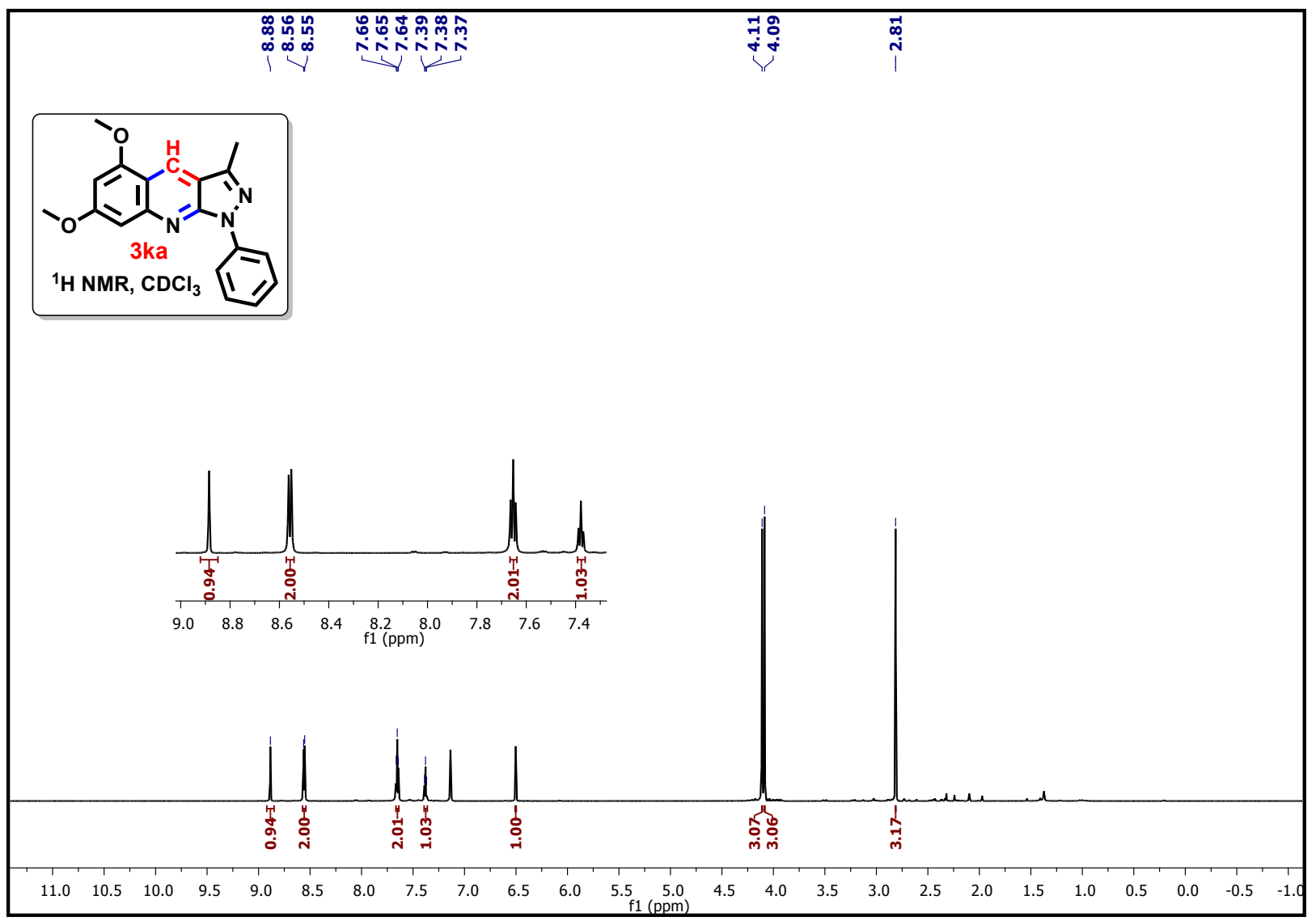

${ }^{13} \mathrm{C}\left\{{ }^{1} \mathrm{H}\right\}$ NMR (200 MHz) spectra of 3ka

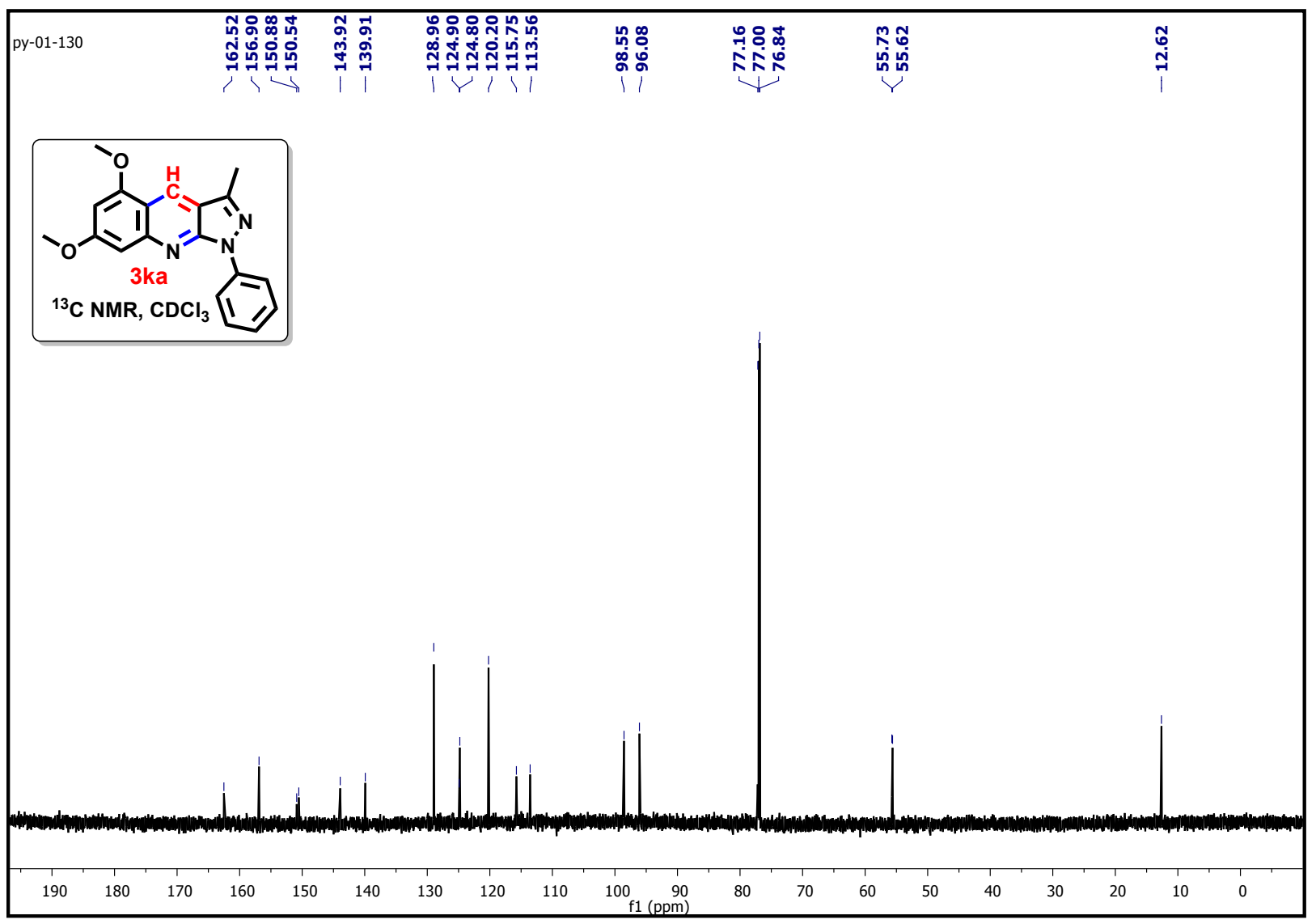


${ }^{1} \mathrm{H}$ NMR (400 MHz) spectra of 3la

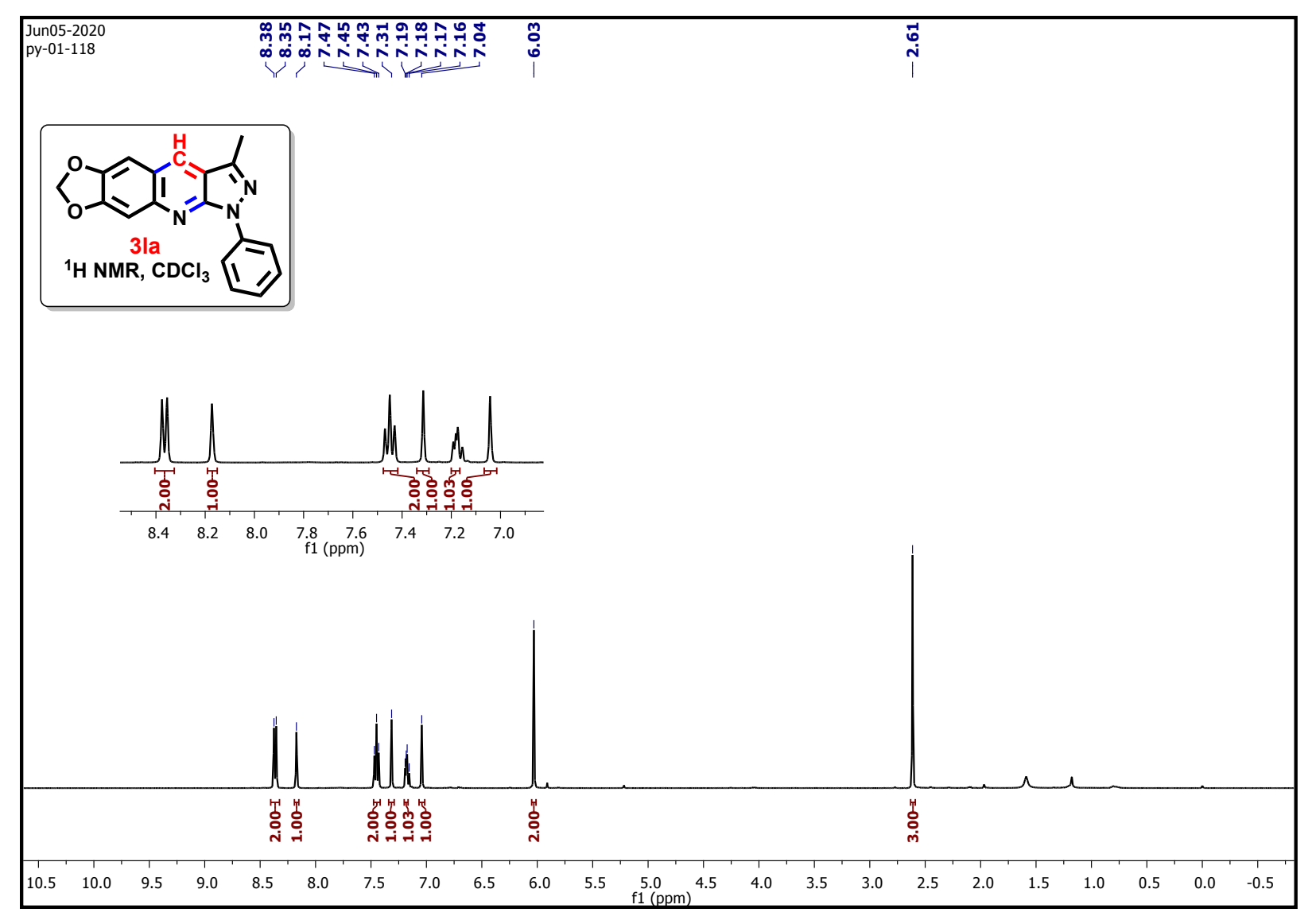

${ }^{13} \mathrm{C}\left\{{ }^{1} \mathrm{H}\right\}$ NMR (101 MHz) spectra of 3la

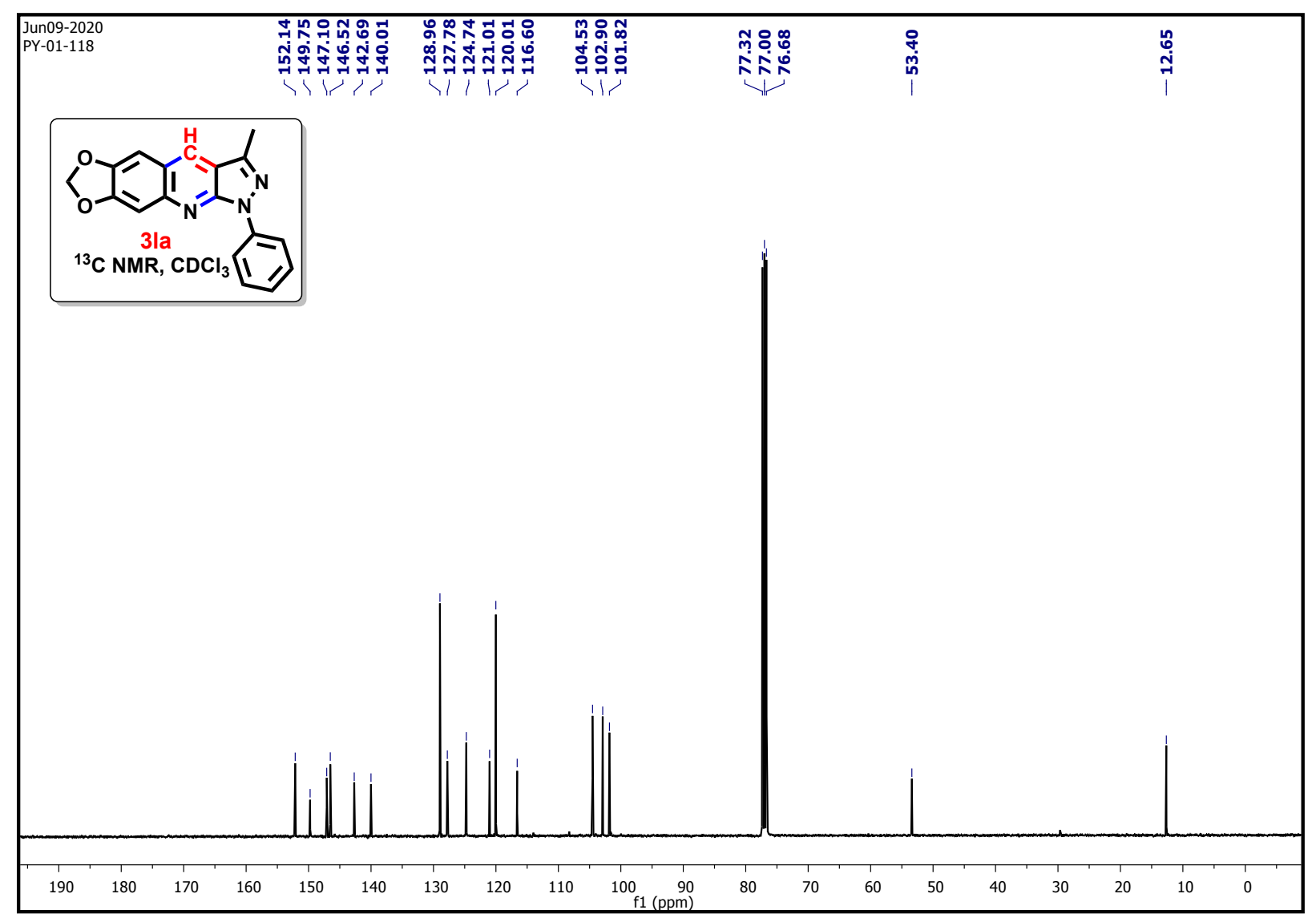


${ }^{1} \mathrm{H}$ NMR (400 MHz) spectra of 3ma

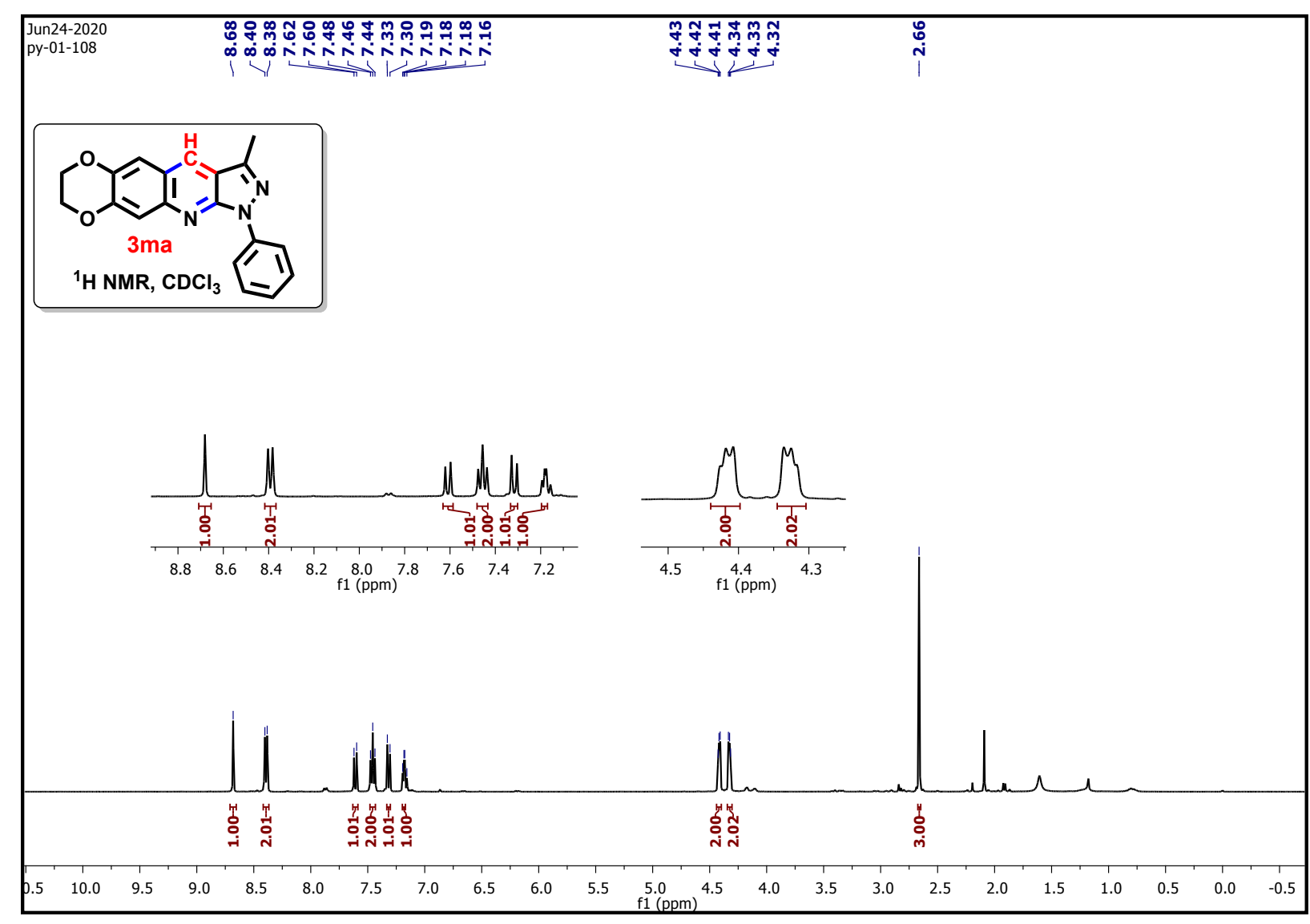

${ }^{13} \mathrm{C}\left\{{ }^{1} \mathrm{H}\right\}$ NMR (101 MHz) spectra of $\mathbf{3 m a}$

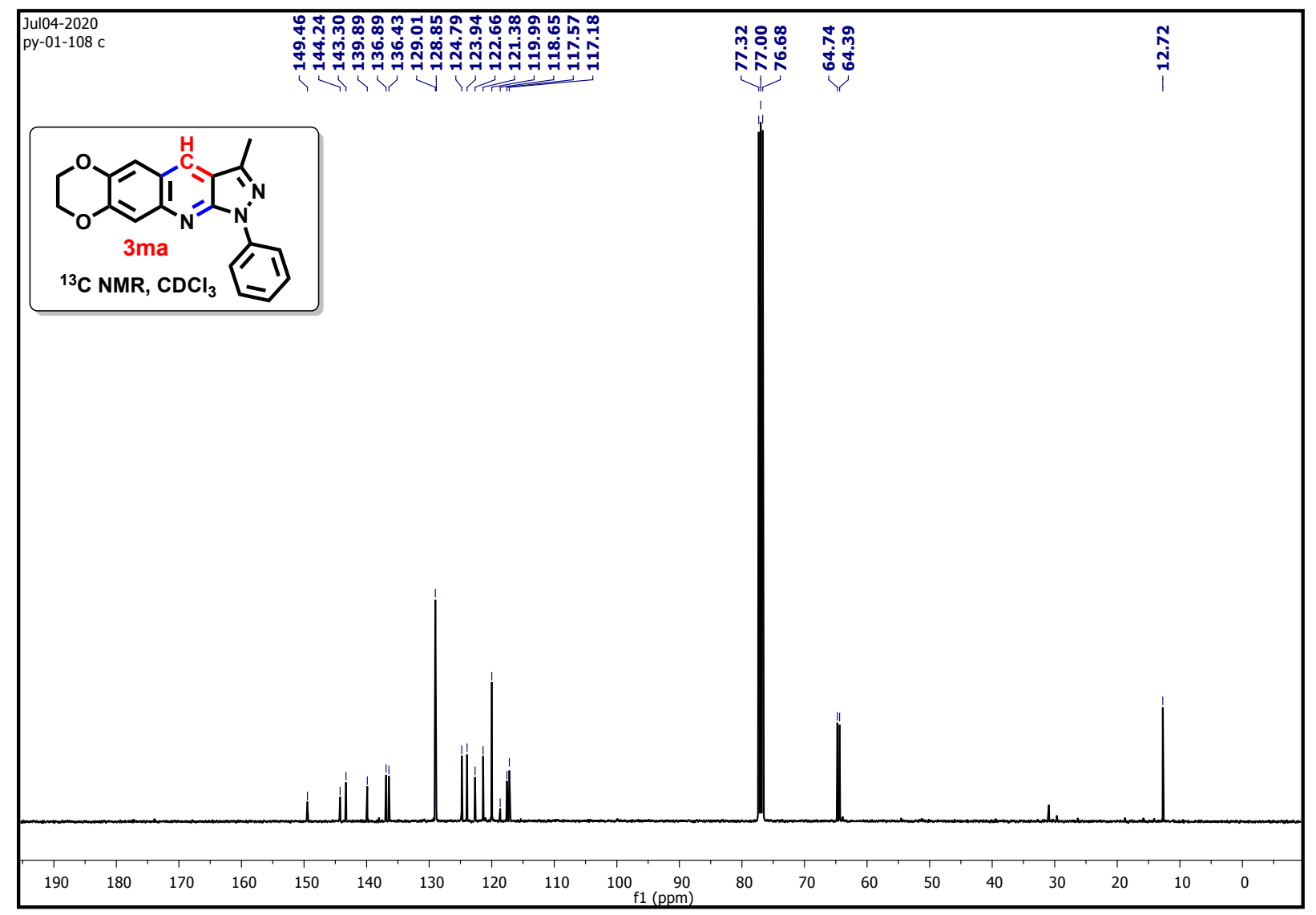


${ }^{1} \mathrm{H}$ NMR $(400 \mathrm{MHz})$ spectra of 3 na

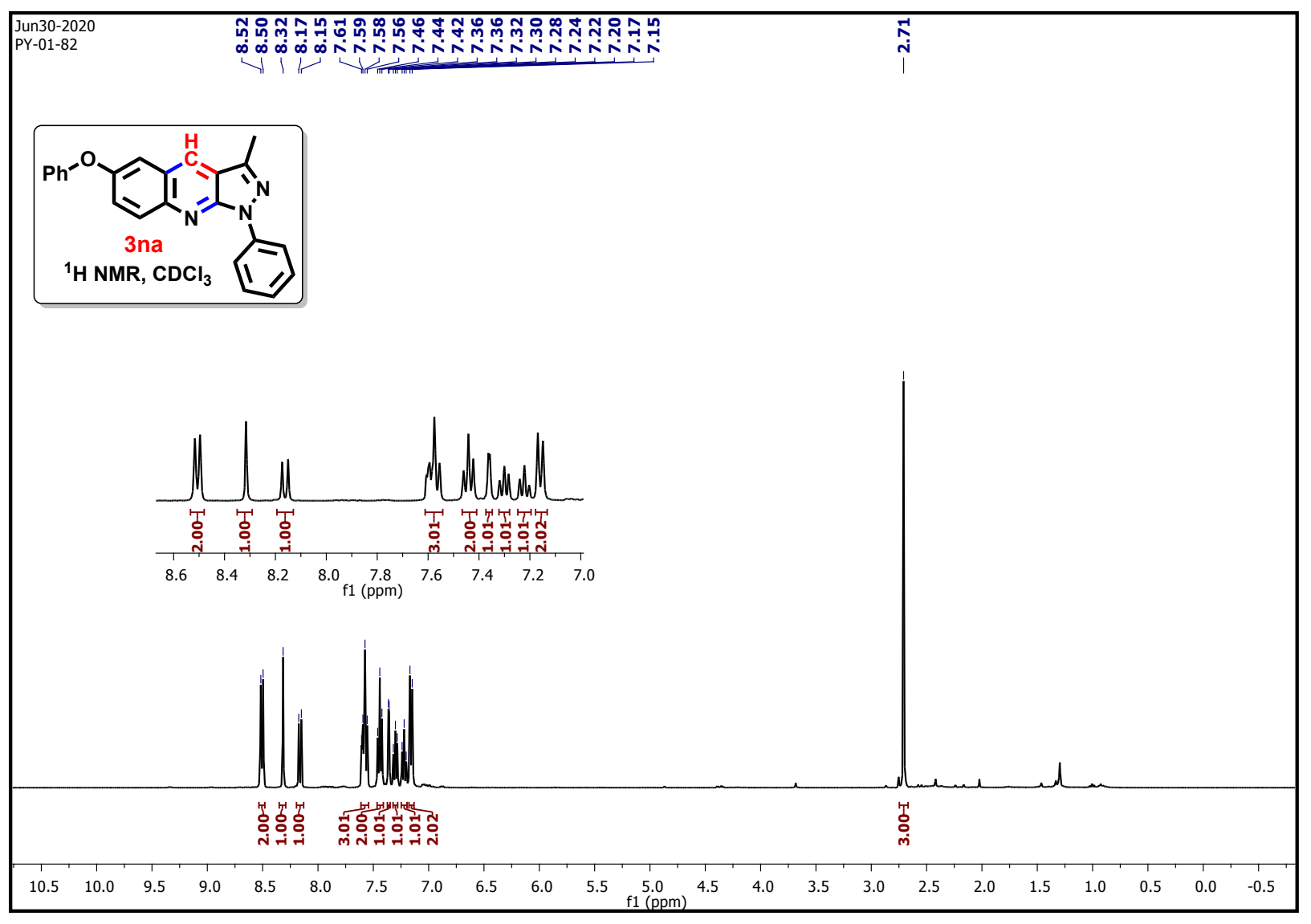

${ }^{13} \mathrm{C}\left\{{ }^{1} \mathrm{H}\right\}$ NMR (101 MHz) spectra of 3na

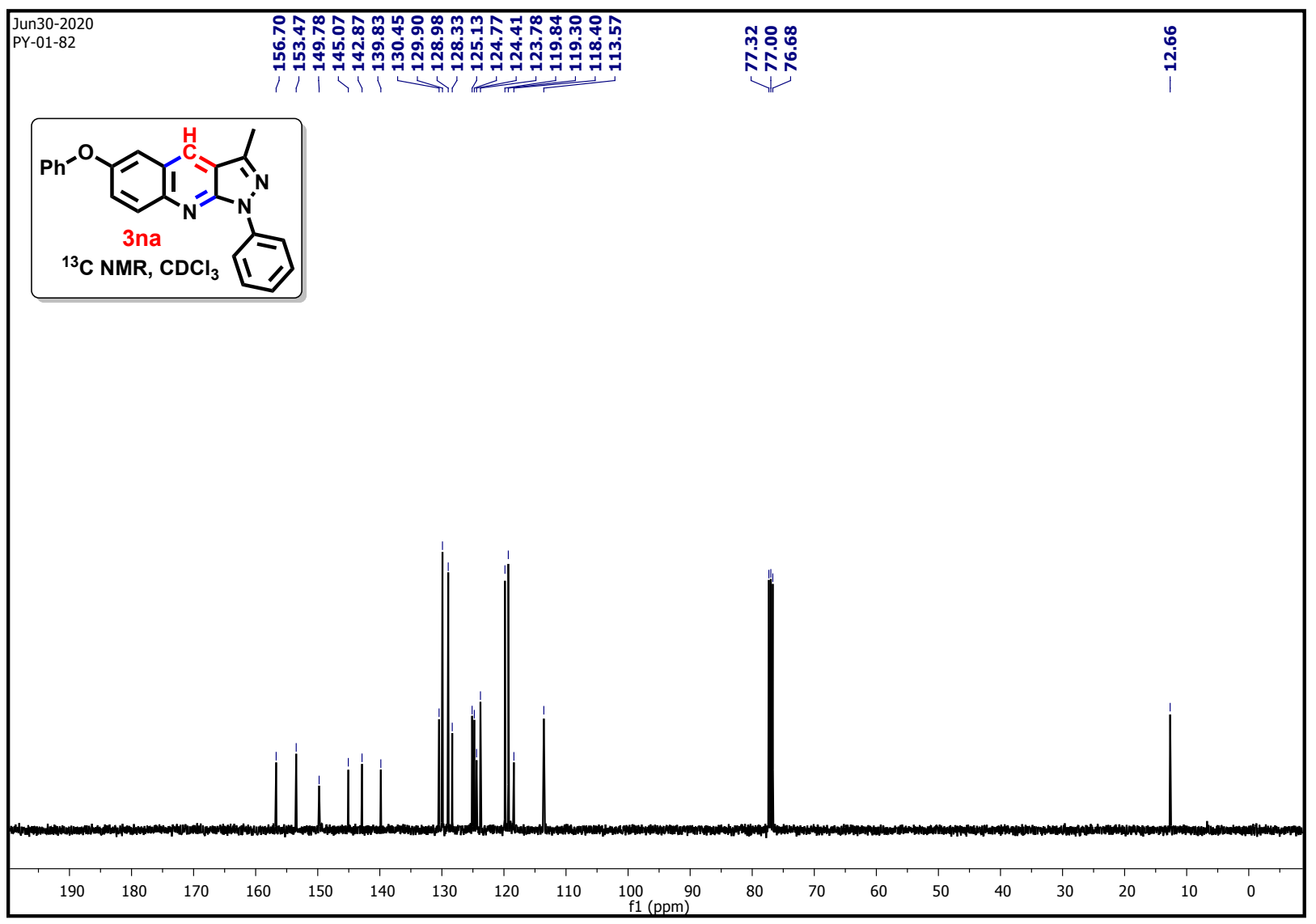


${ }^{1} \mathrm{H}$ NMR (400 MHz) spectra of $30 a$

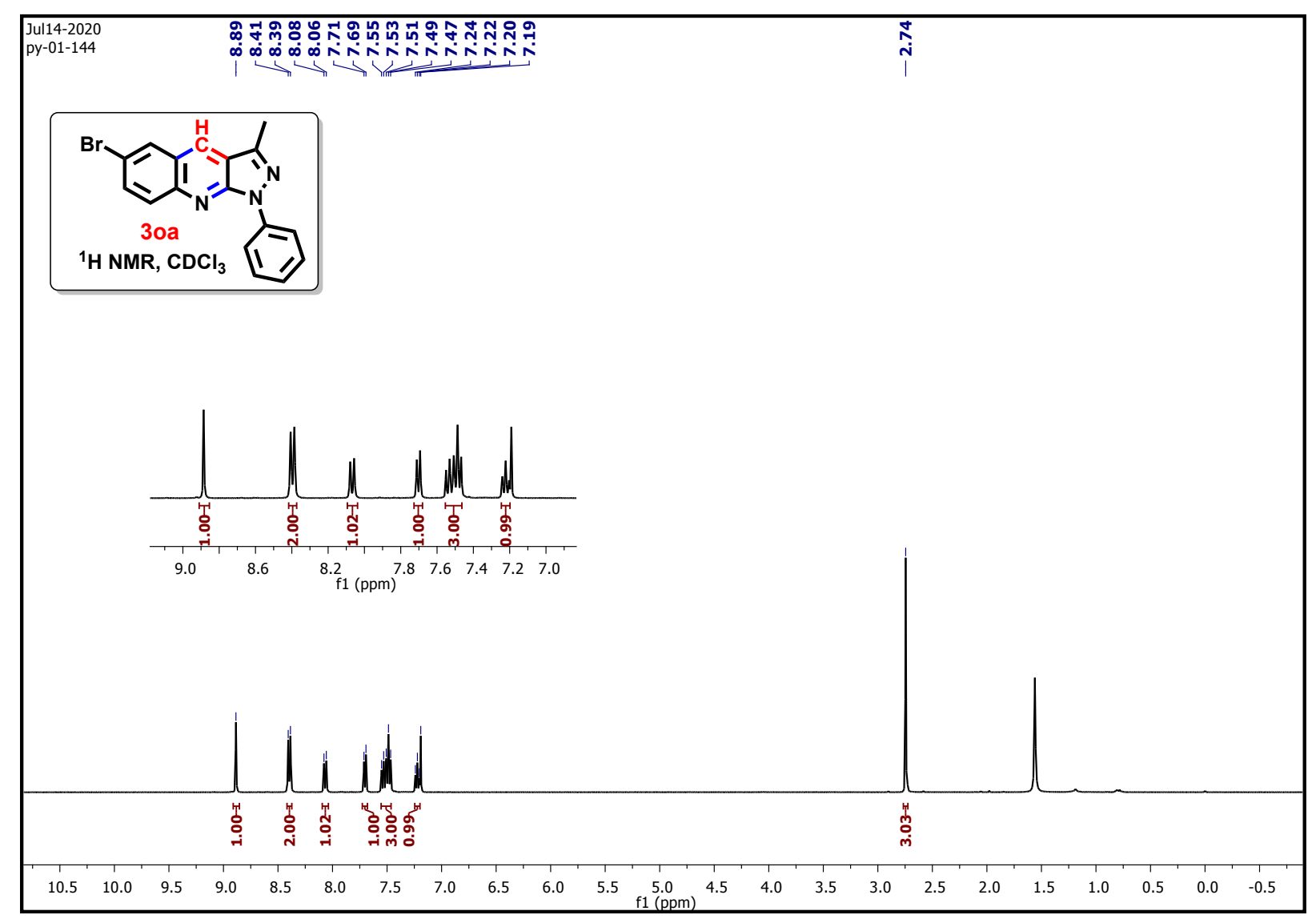

${ }^{13} \mathrm{C}\left\{{ }^{1} \mathrm{H}\right\}$ NMR (101 MHz) spectra of $\mathbf{3 0 a}$

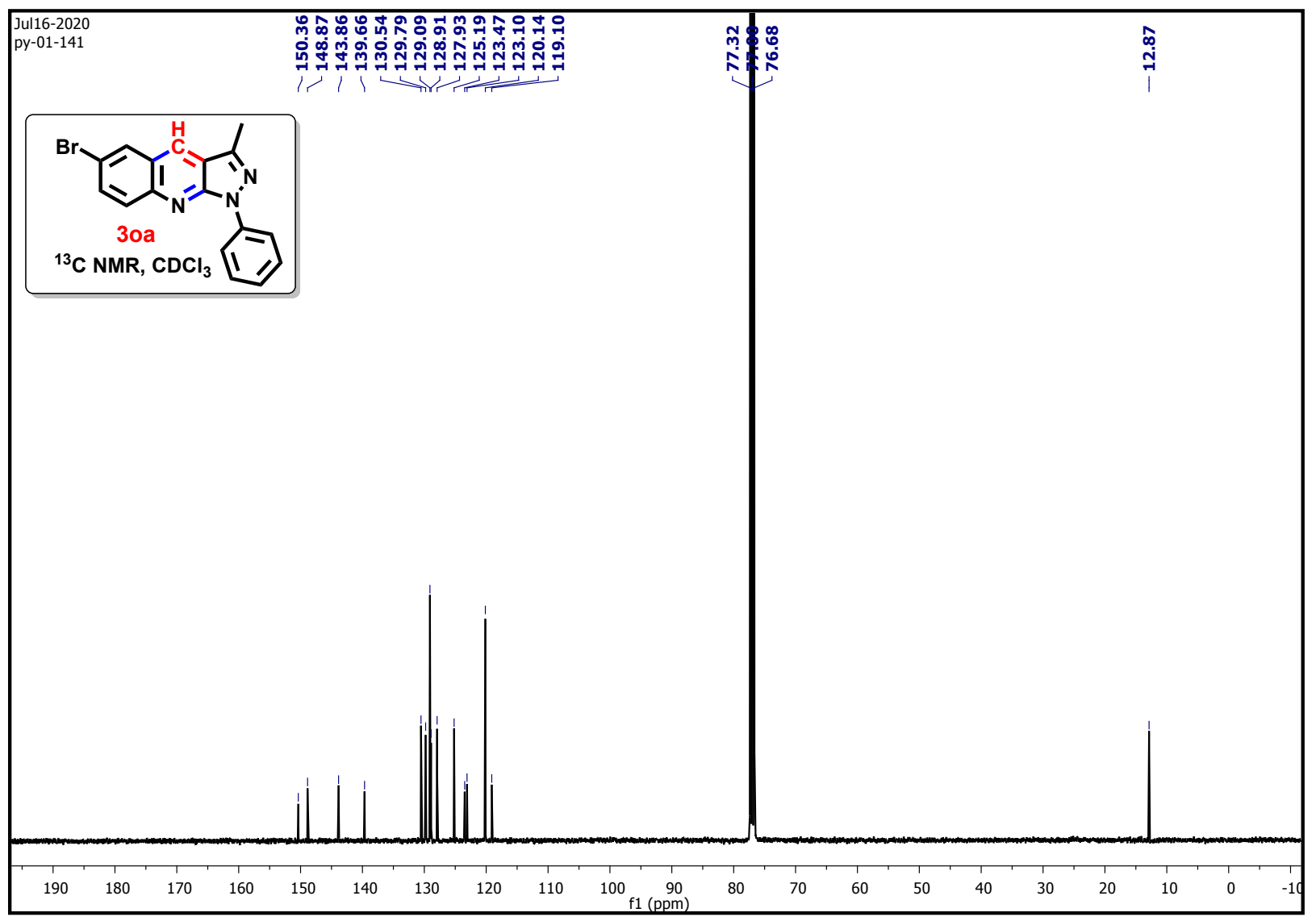


${ }^{1} \mathrm{H}$ NMR $(400 \mathrm{MHz})$ spectra of $3 p a$

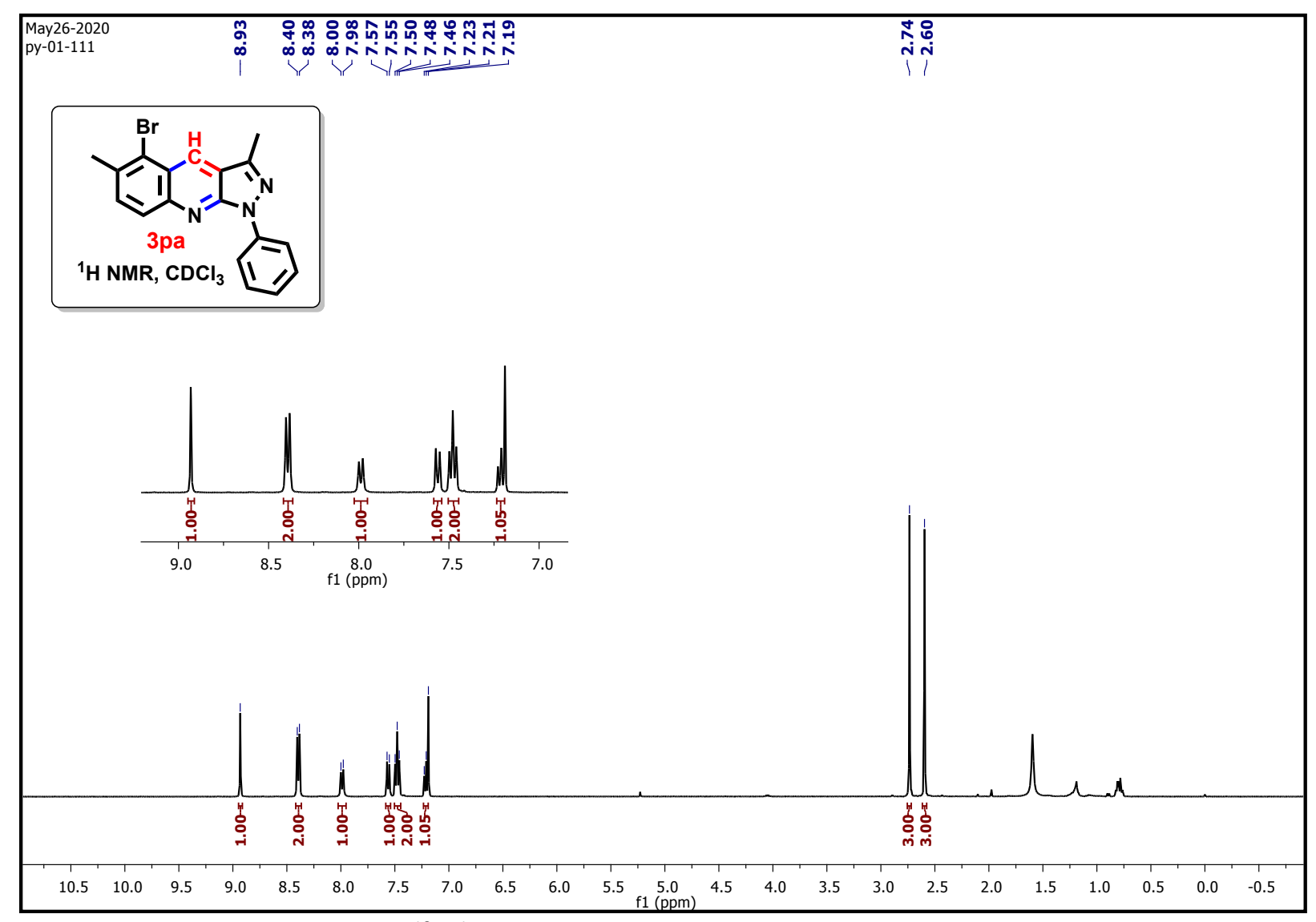

${ }^{13} \mathrm{C}\left\{{ }^{1} \mathrm{H}\right\}$ NMR (101 MHz) spectra of 3pa

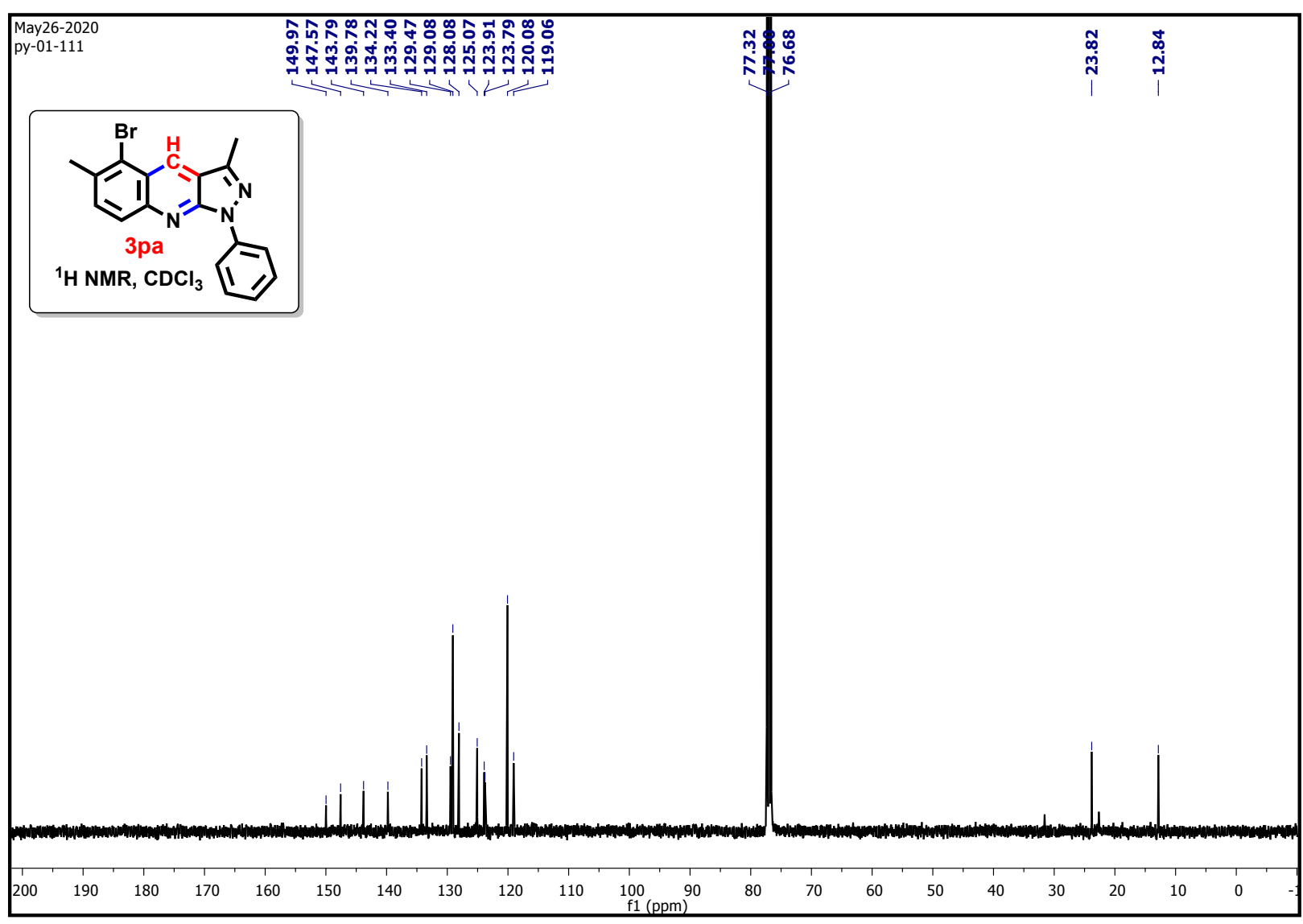


${ }^{1} \mathrm{H}$ NMR (400 MHz) spectra of 3qa

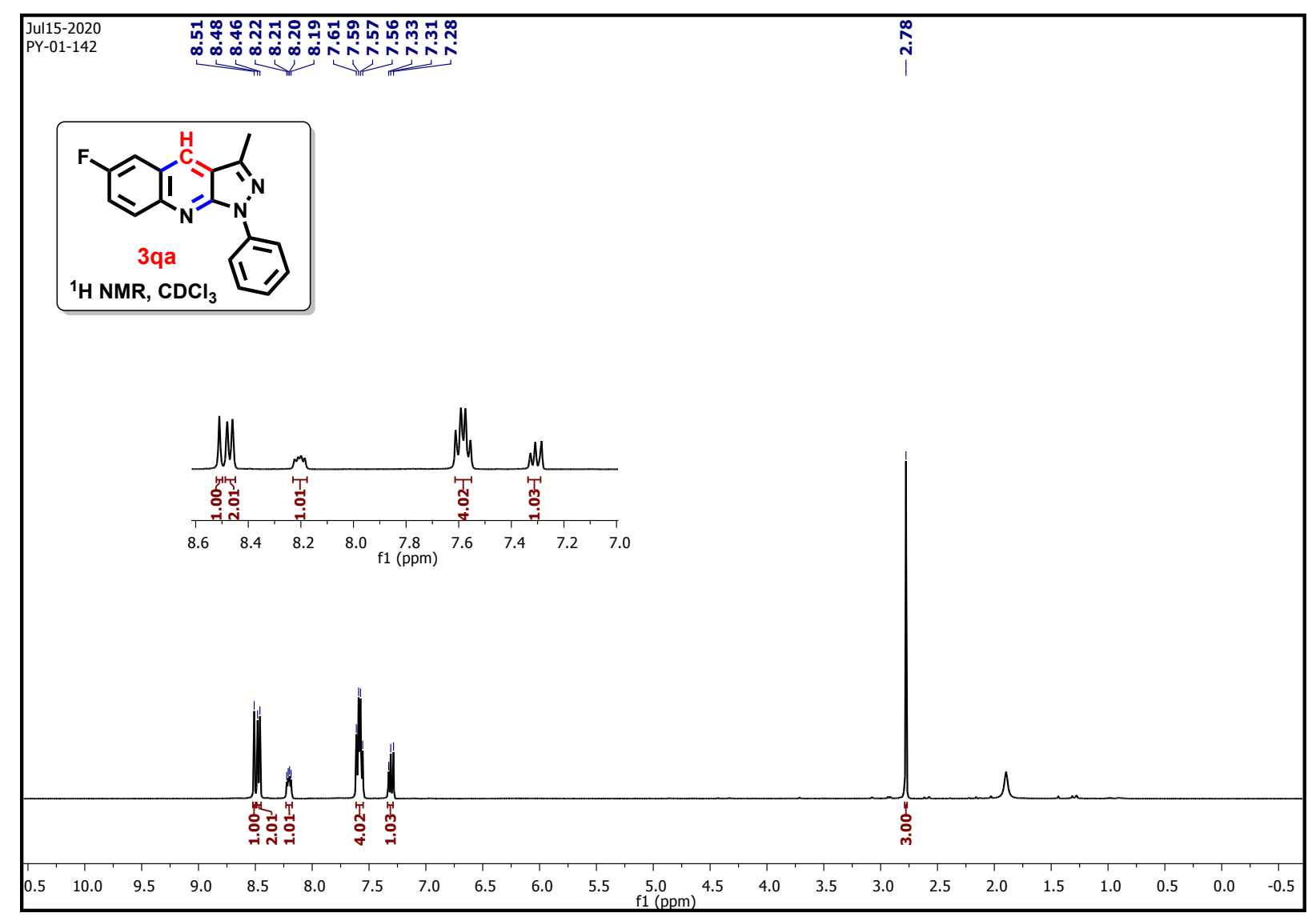

${ }^{13} \mathrm{C}\left\{{ }^{1} \mathrm{H}\right\}$ NMR (101 MHz) spectra of 3qa

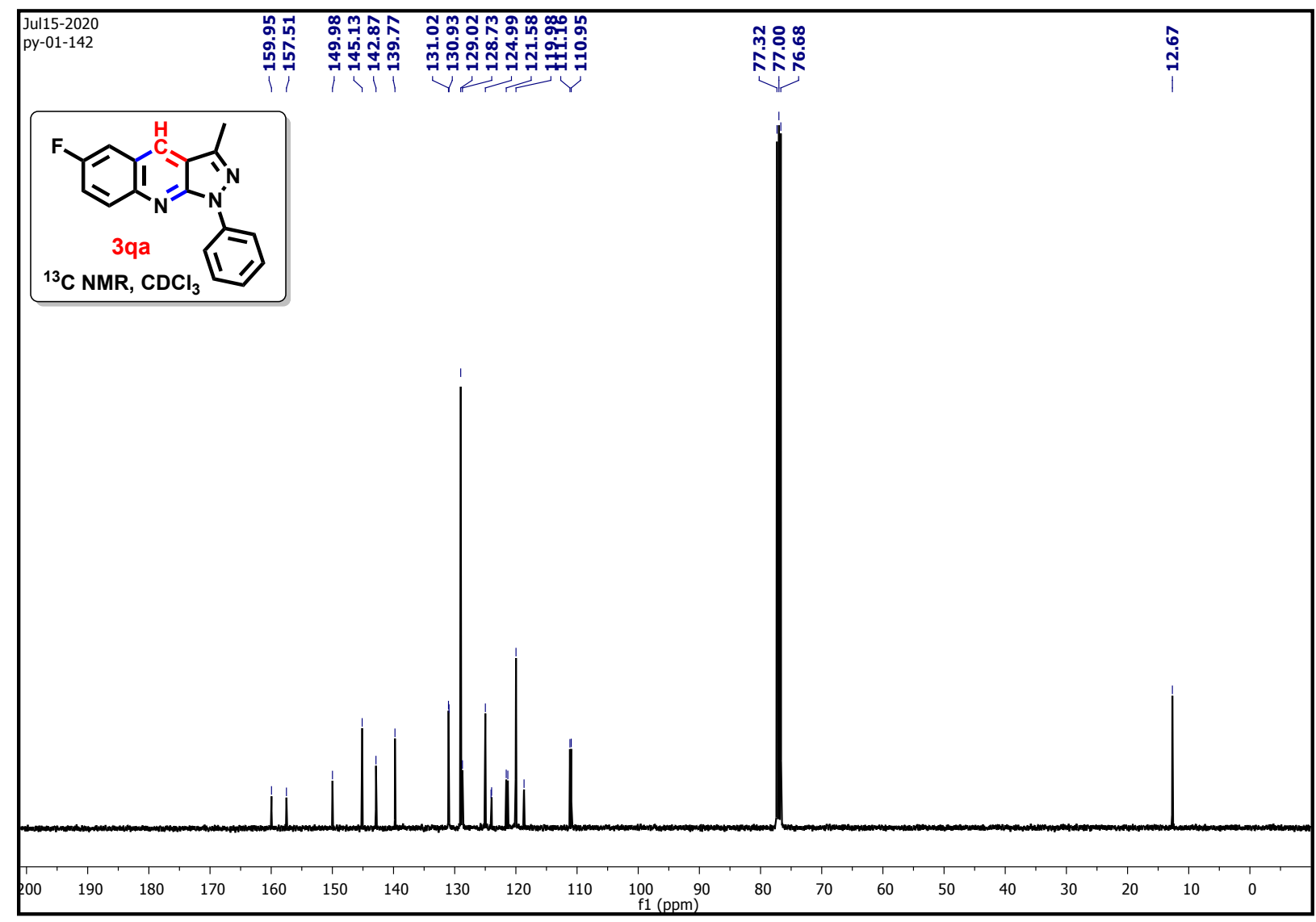


${ }^{1} \mathrm{H}$ NMR (400 MHz) spectra of 3ra

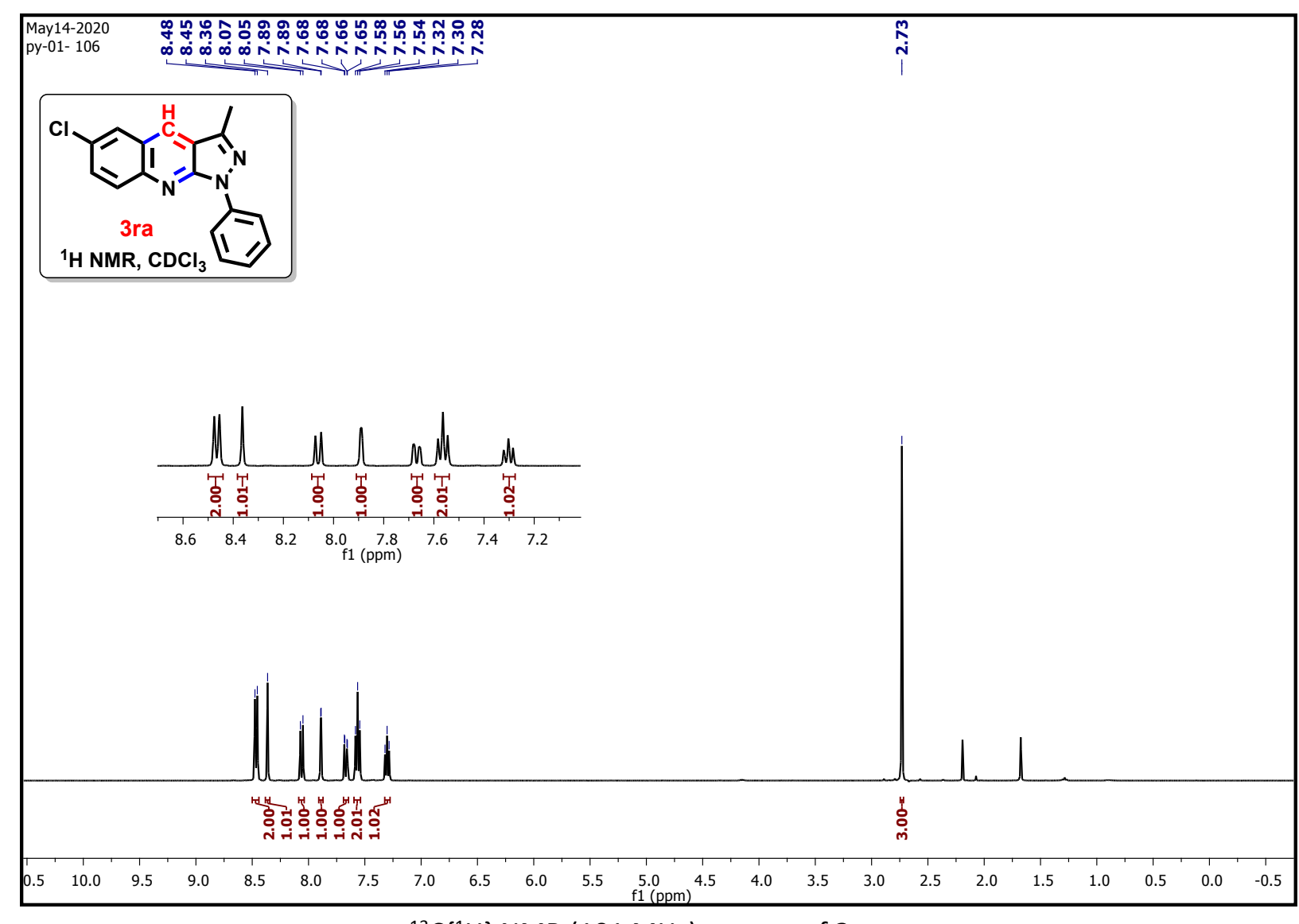

${ }^{13} \mathrm{C}\left\{{ }^{1} \mathrm{H}\right\}$ NMR $(101 \mathrm{MHz})$ spectra of 3ra

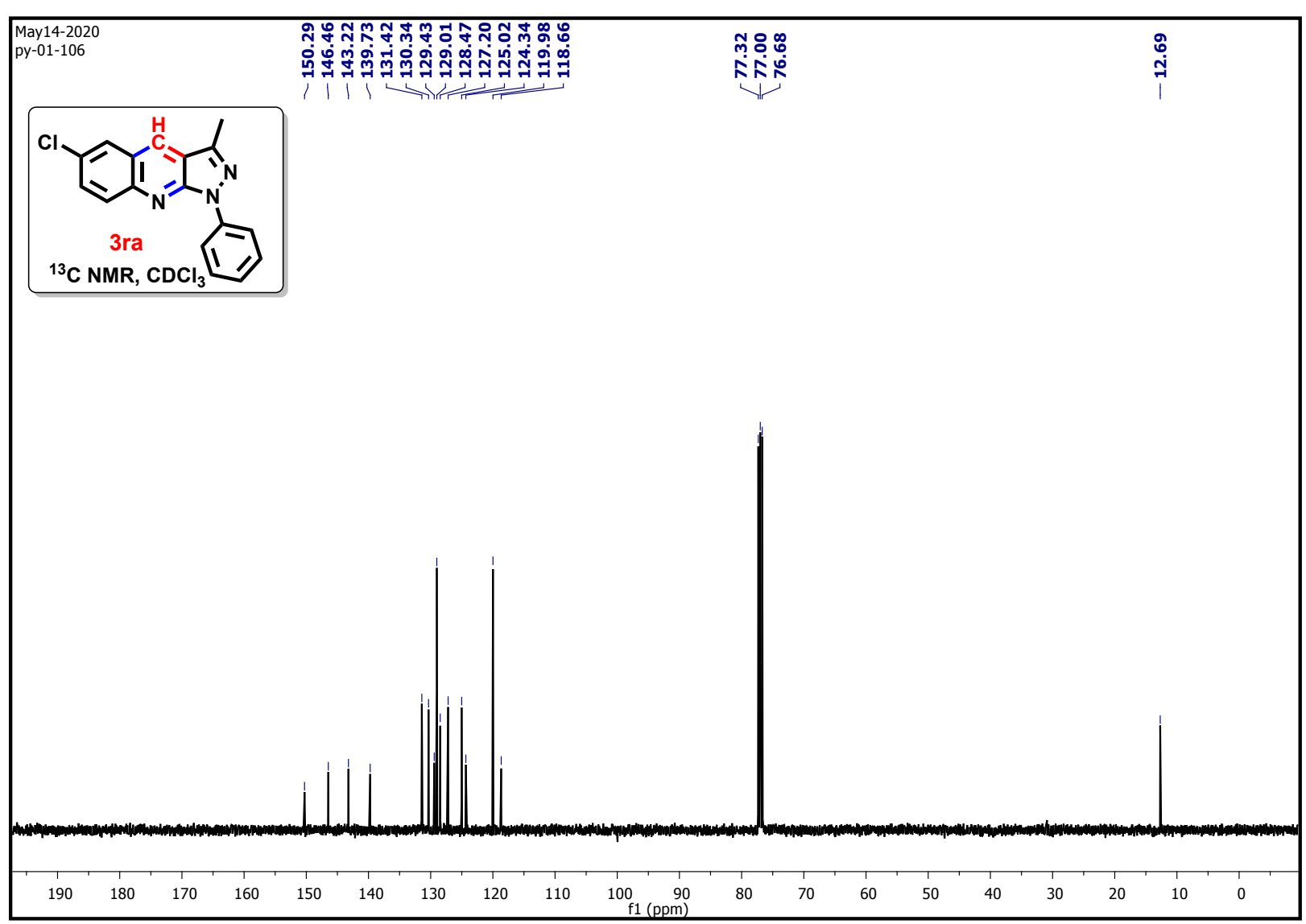


${ }^{1} \mathrm{H}$ NMR (400 MHz) spectra of 3sa

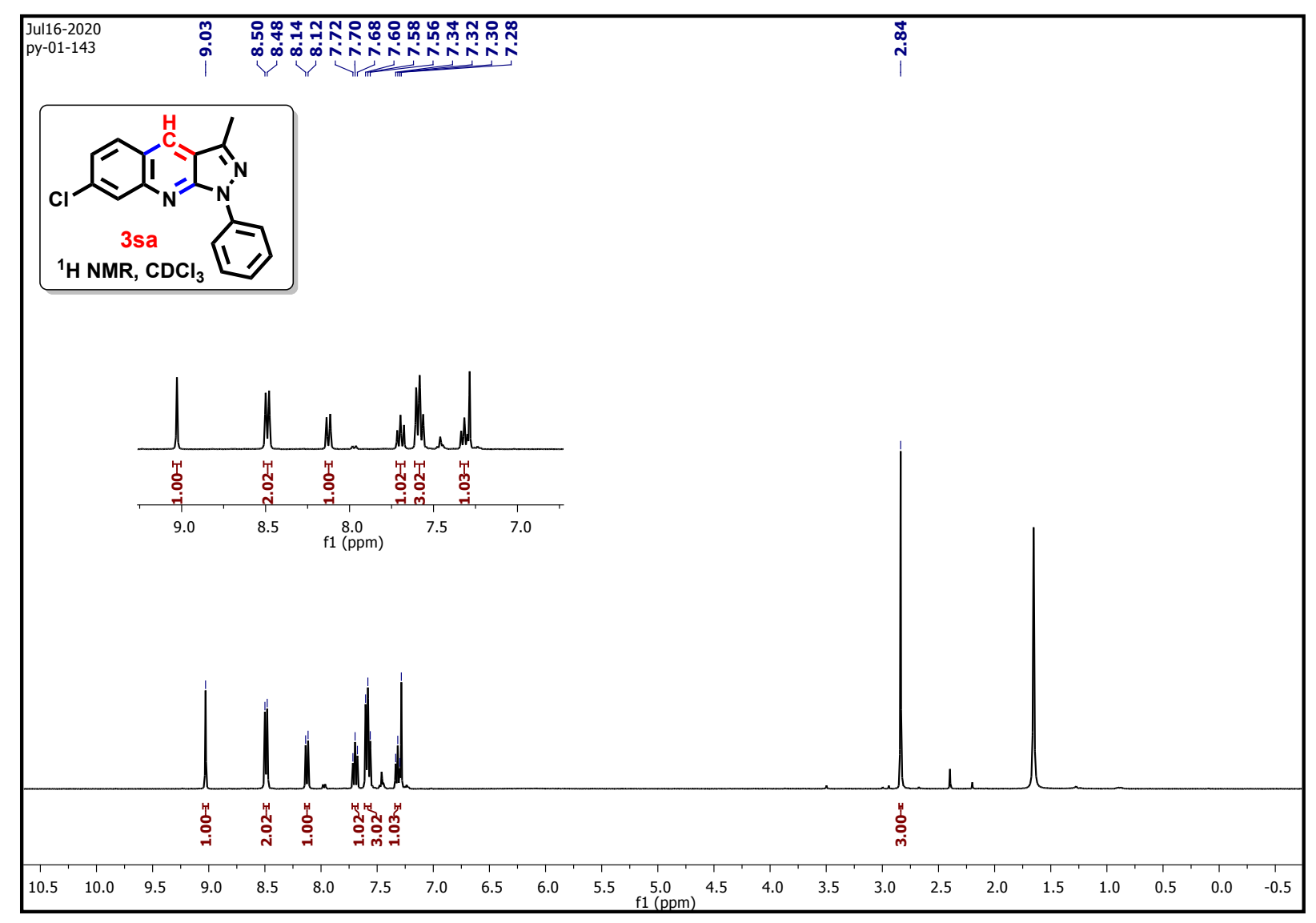

${ }^{13} \mathrm{C}\left\{{ }^{1} \mathrm{H}\right\}$ NMR (101 MHz) spectra of 3sa

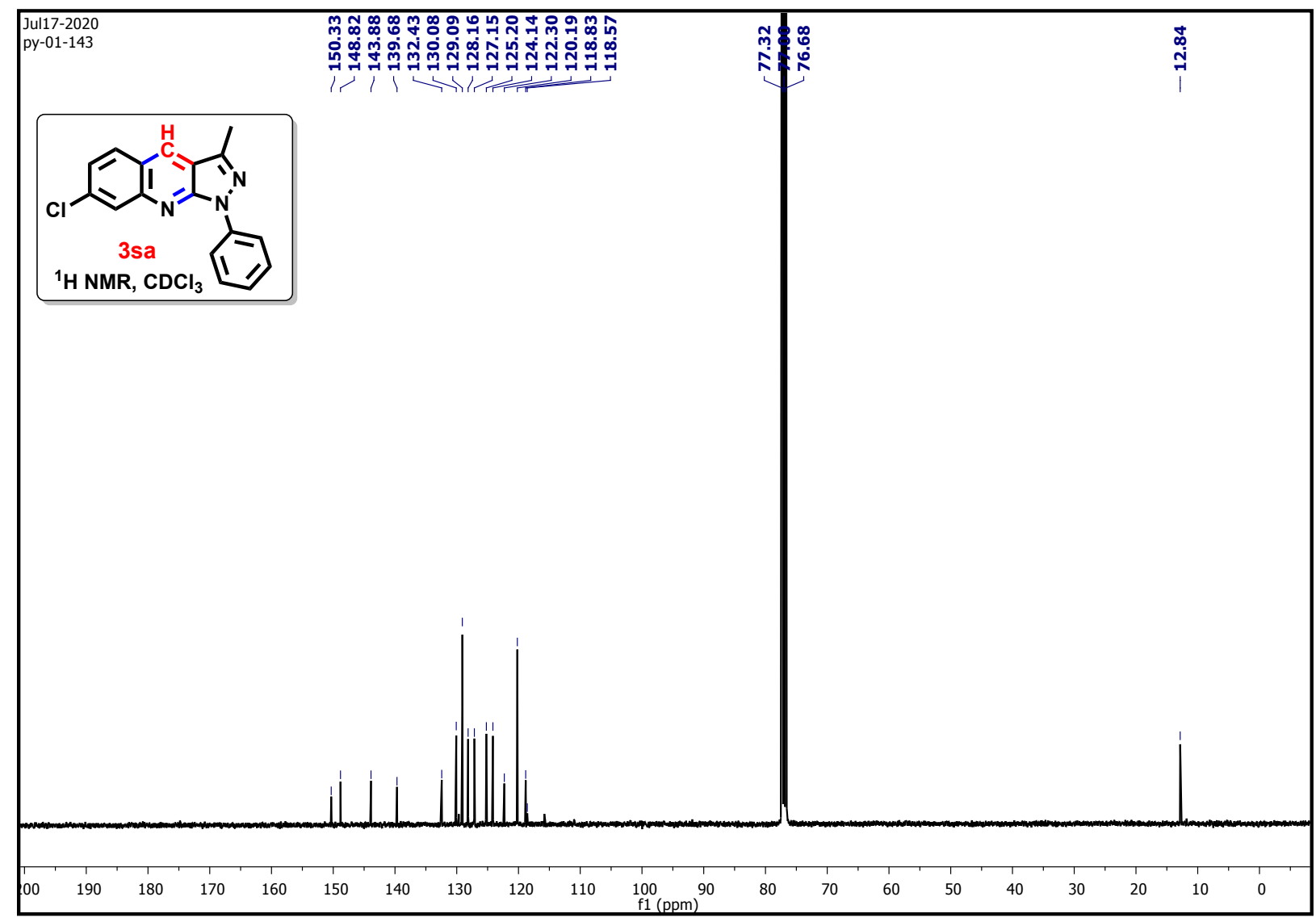


${ }^{1} \mathrm{H}$ NMR (400 MHz) spectra of $3 a b$

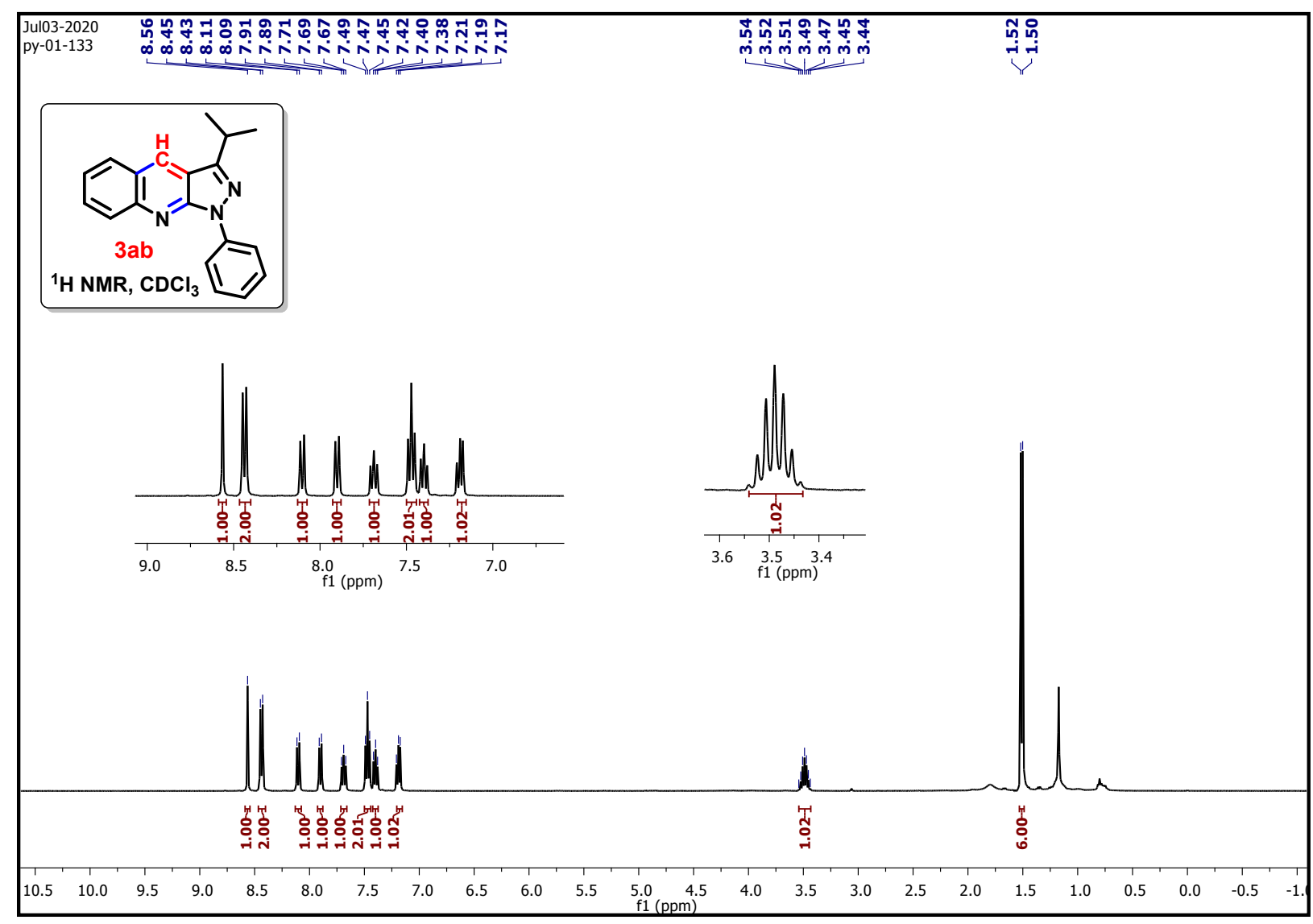

${ }^{13} \mathrm{C}\left\{{ }^{1} \mathrm{H}\right\}$ NMR (101 MHz) spectra of $\mathbf{3 a b}$

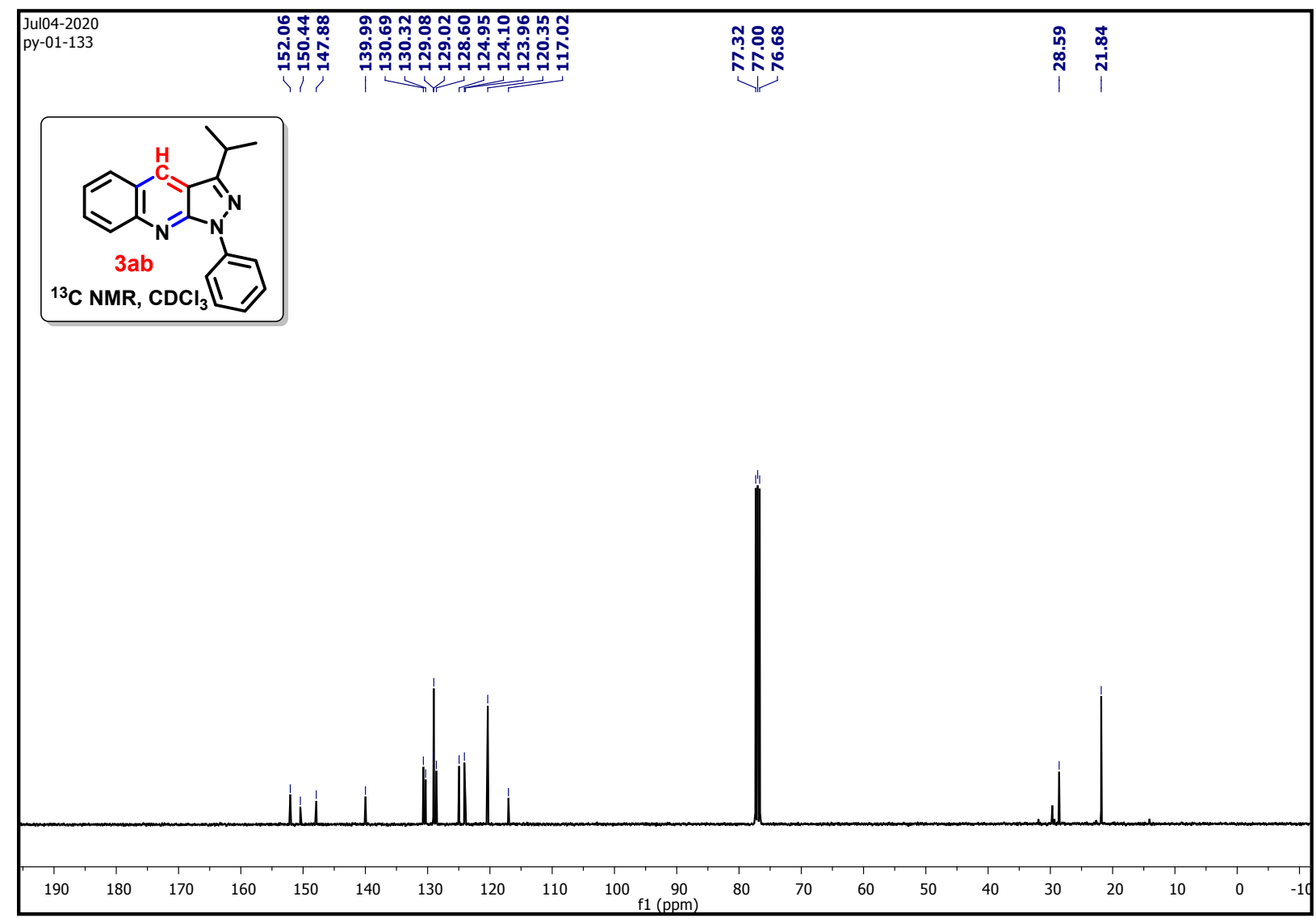


${ }^{1} \mathrm{H}$ NMR (400 MHz) spectra of $\mathbf{3 b b}$

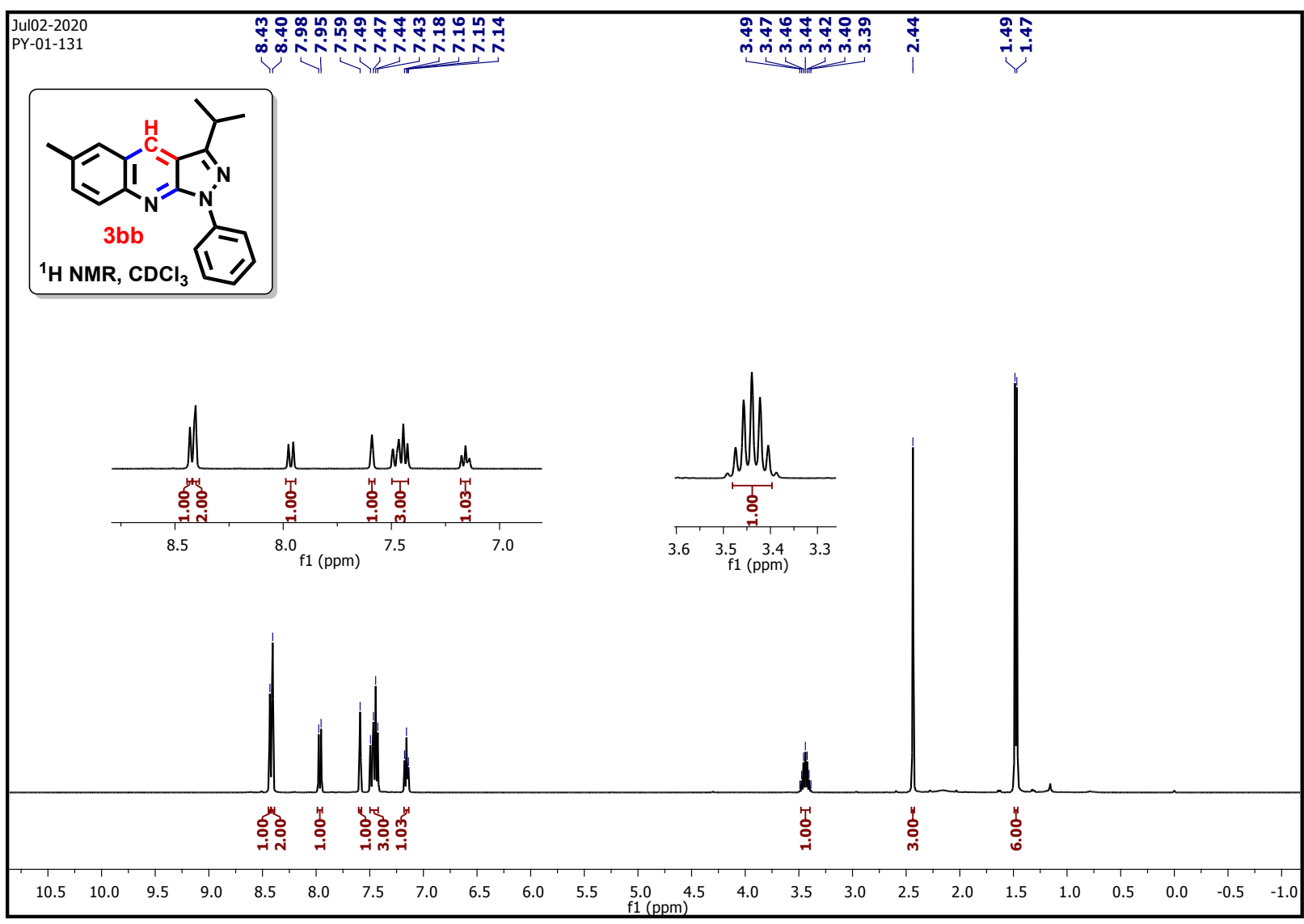

${ }^{13} \mathrm{C}\left\{{ }^{1} \mathrm{H}\right\}$ NMR (101 MHz) spectra of $\mathbf{3 b} \mathbf{b}$

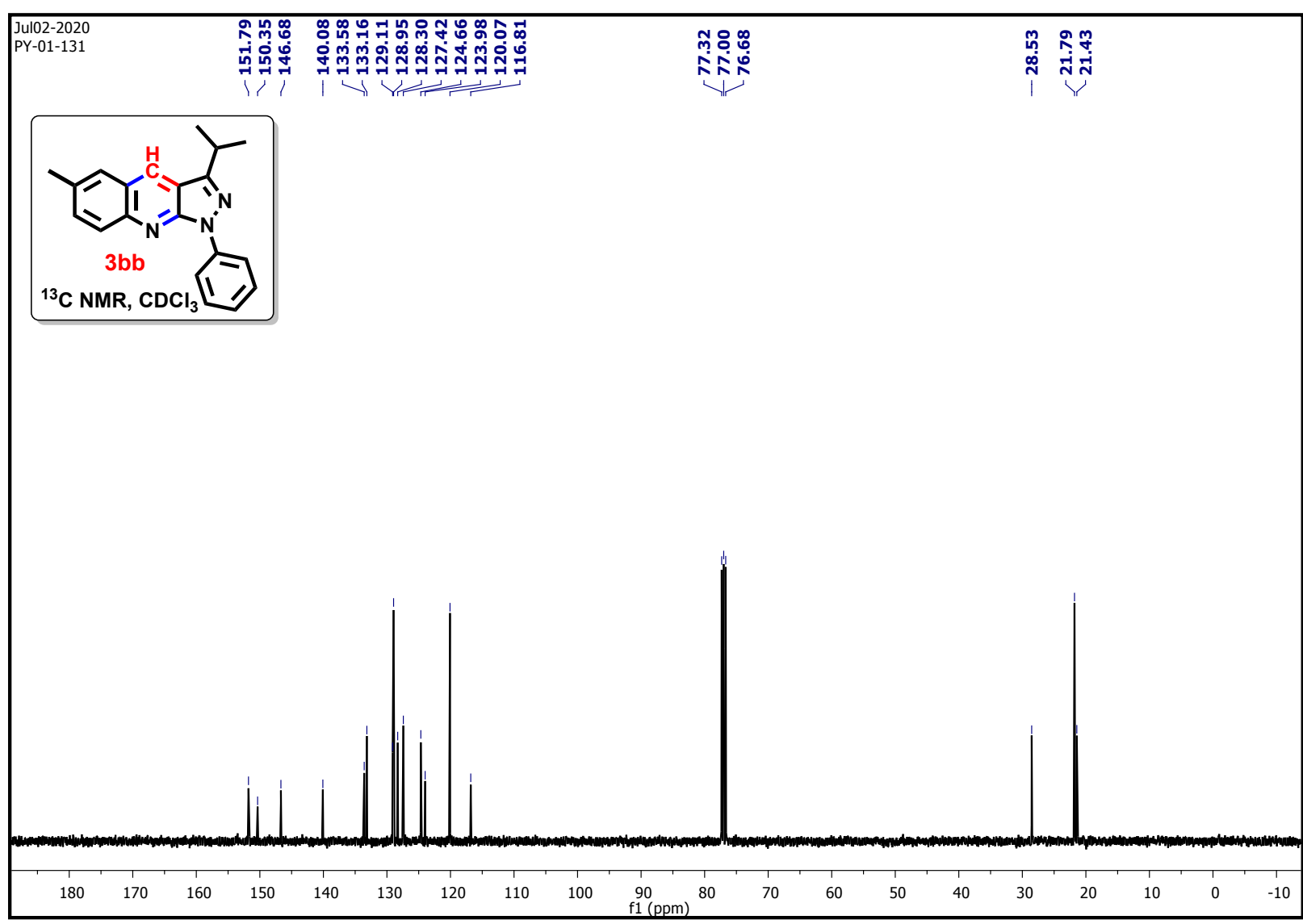


${ }^{1} \mathrm{H}$ NMR (400 MHz) spectra of 3ib

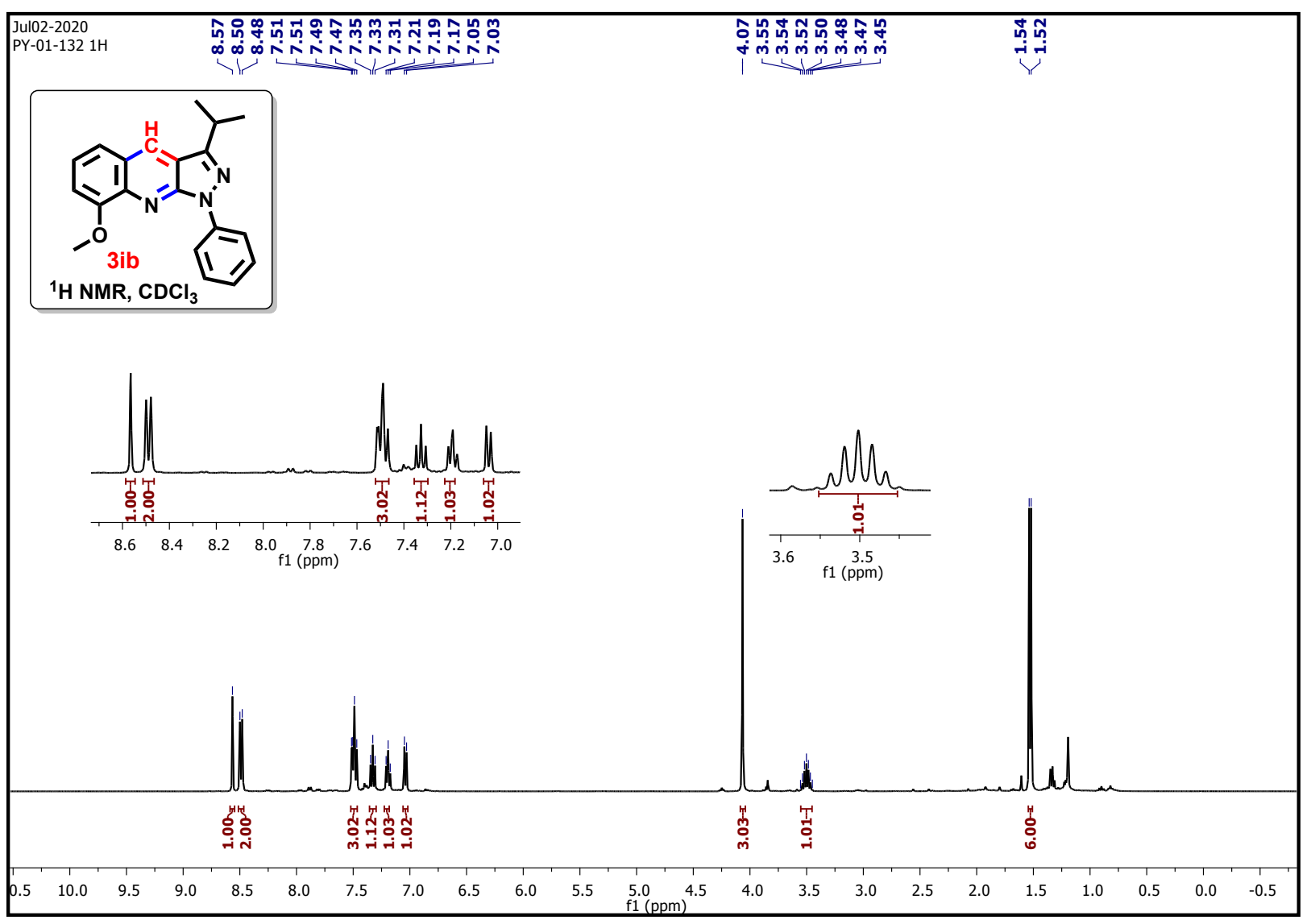

${ }^{13} \mathrm{C}\left\{{ }^{1} \mathrm{H}\right\}$ NMR (101 MHz) spectra of 3ib

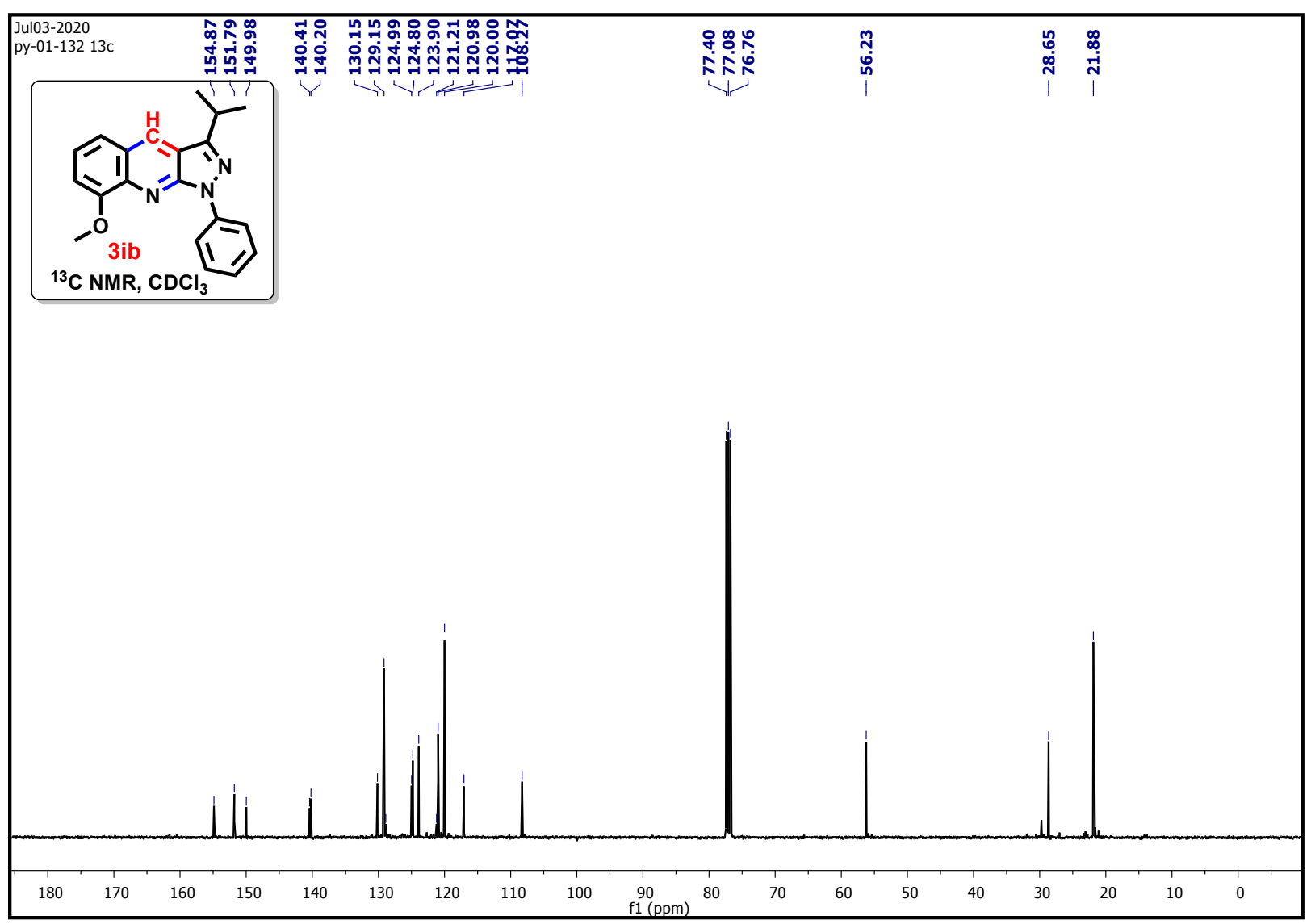


${ }^{1} \mathrm{H}$ NMR (400 MHz) spectra of 3lb

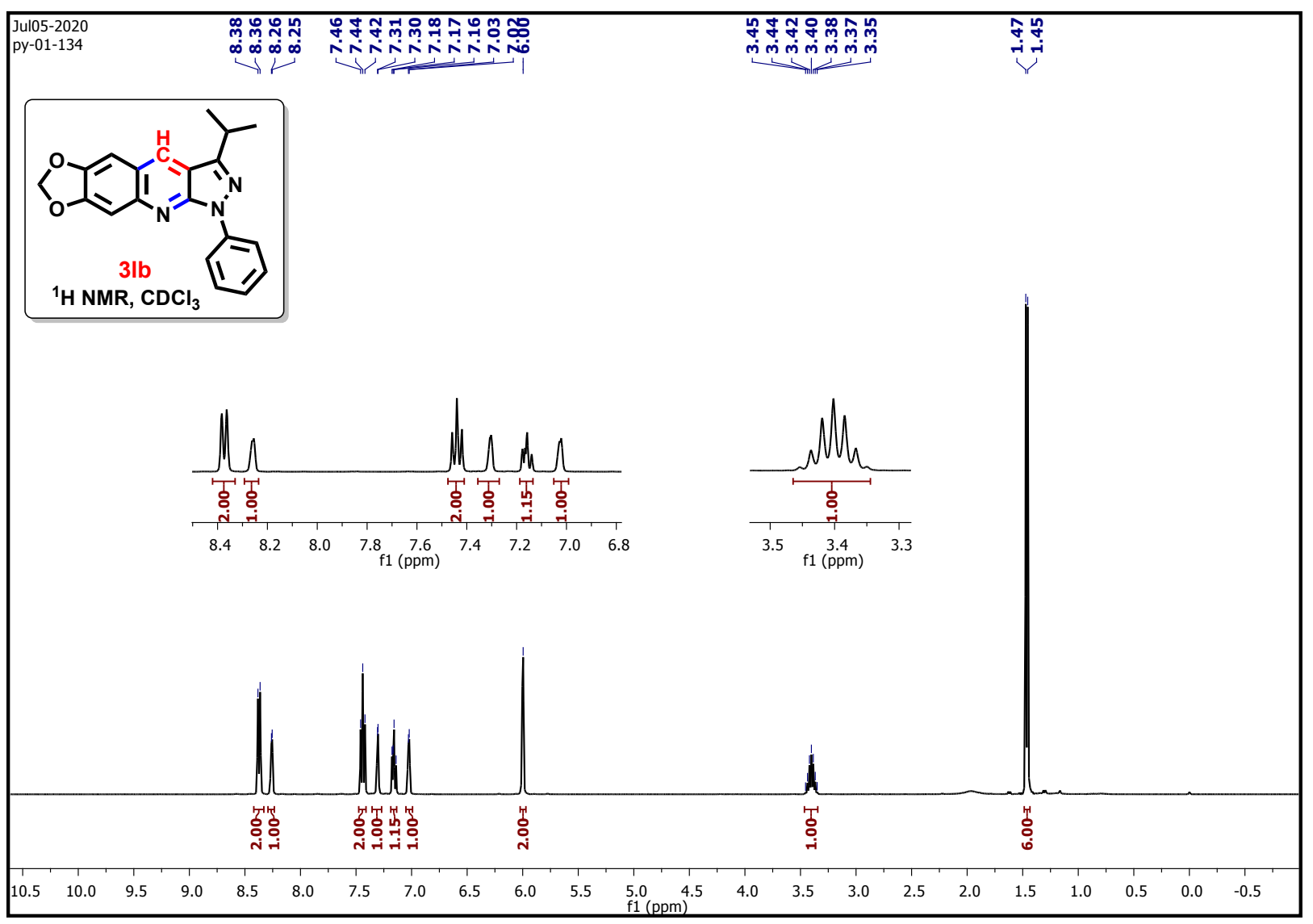

${ }^{13} \mathrm{C}\left\{{ }^{1} \mathrm{H}\right\}$ NMR (101 MHz) spectra of 3/b

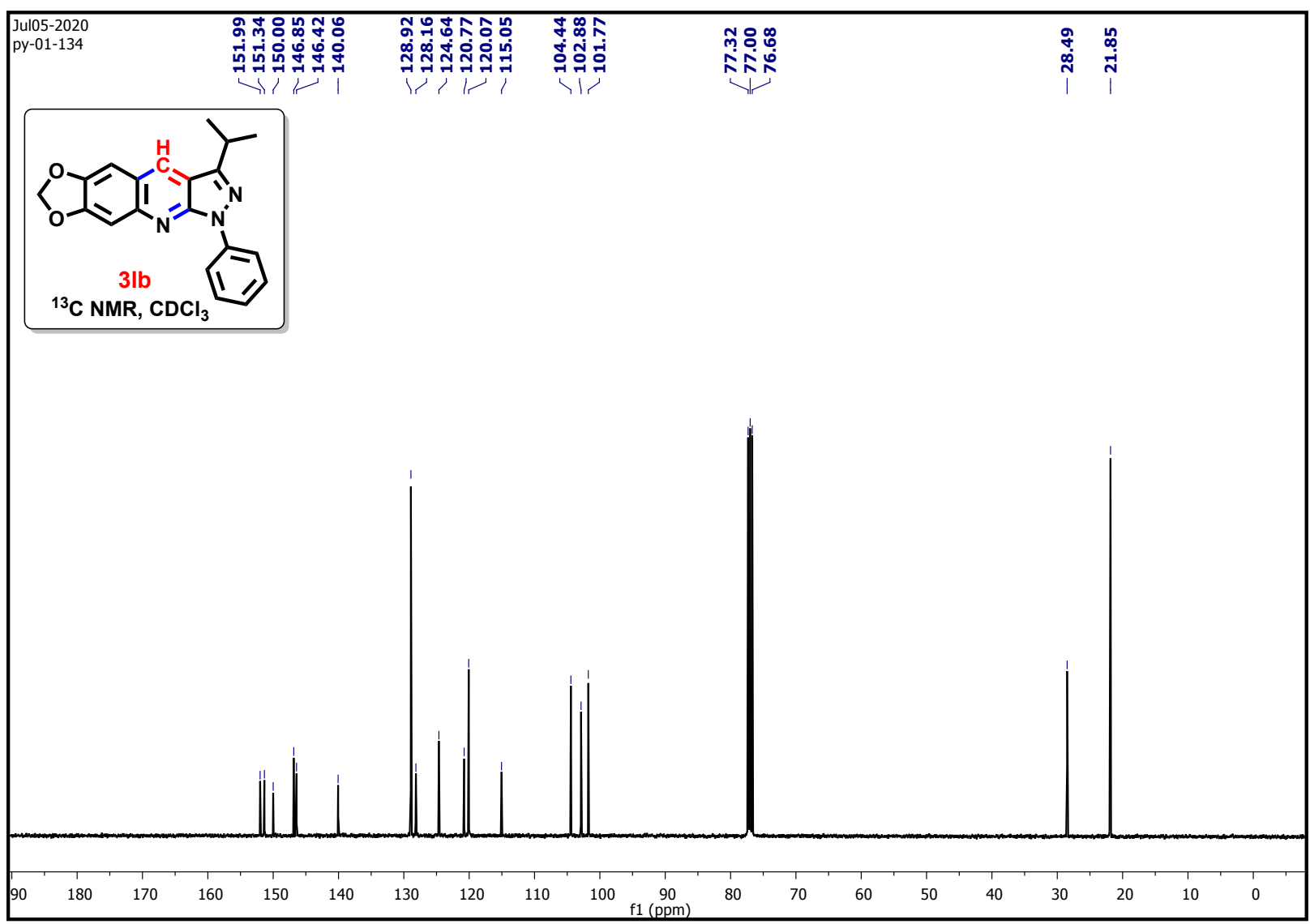


${ }^{1} \mathrm{H}$ NMR $(400 \mathrm{MHz})$ spectra of $\mathbf{3 q b}$

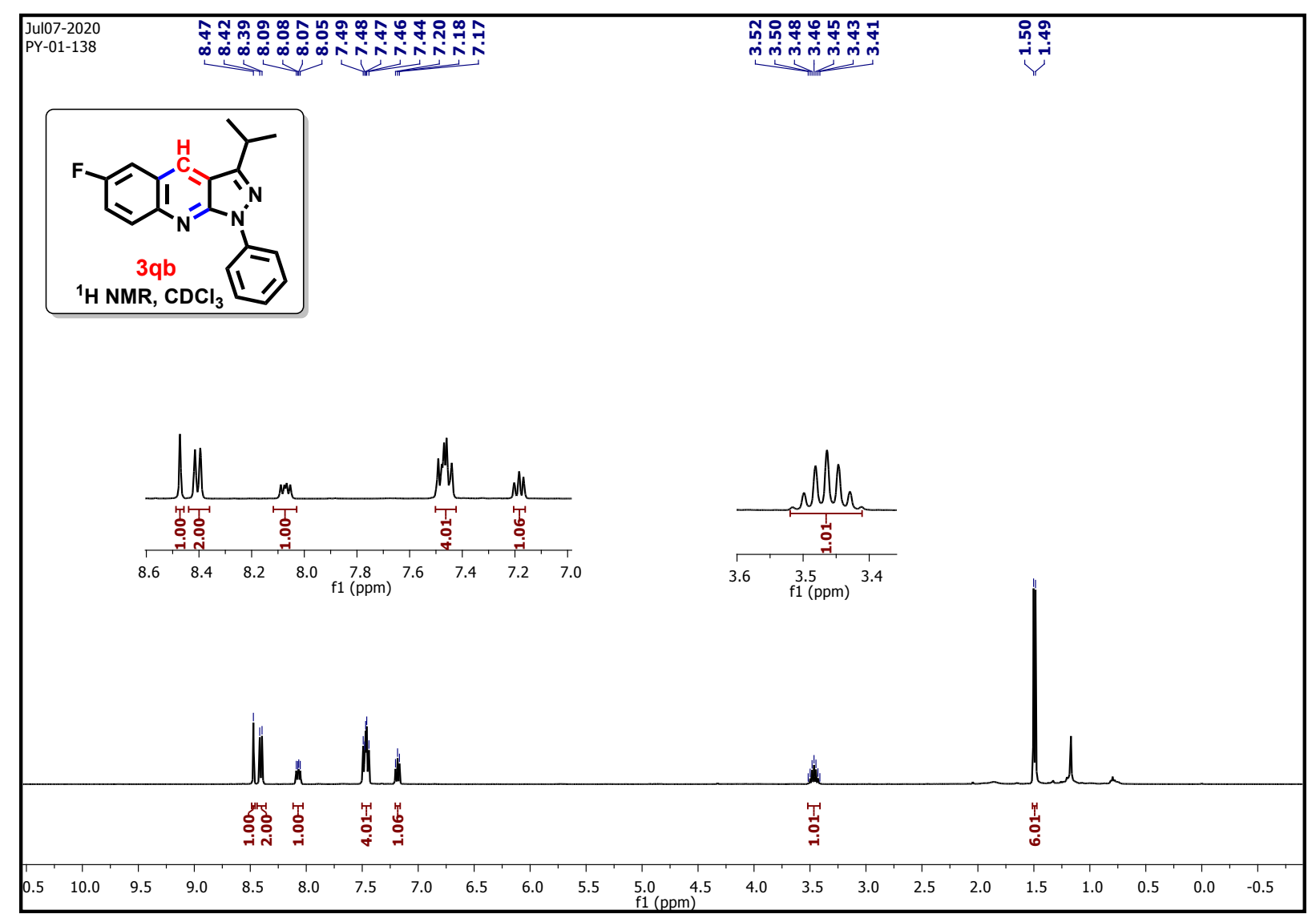

${ }^{13} \mathrm{C}\left\{{ }^{1} \mathrm{H}\right\}$ NMR (101 MHz) spectra of 3qb

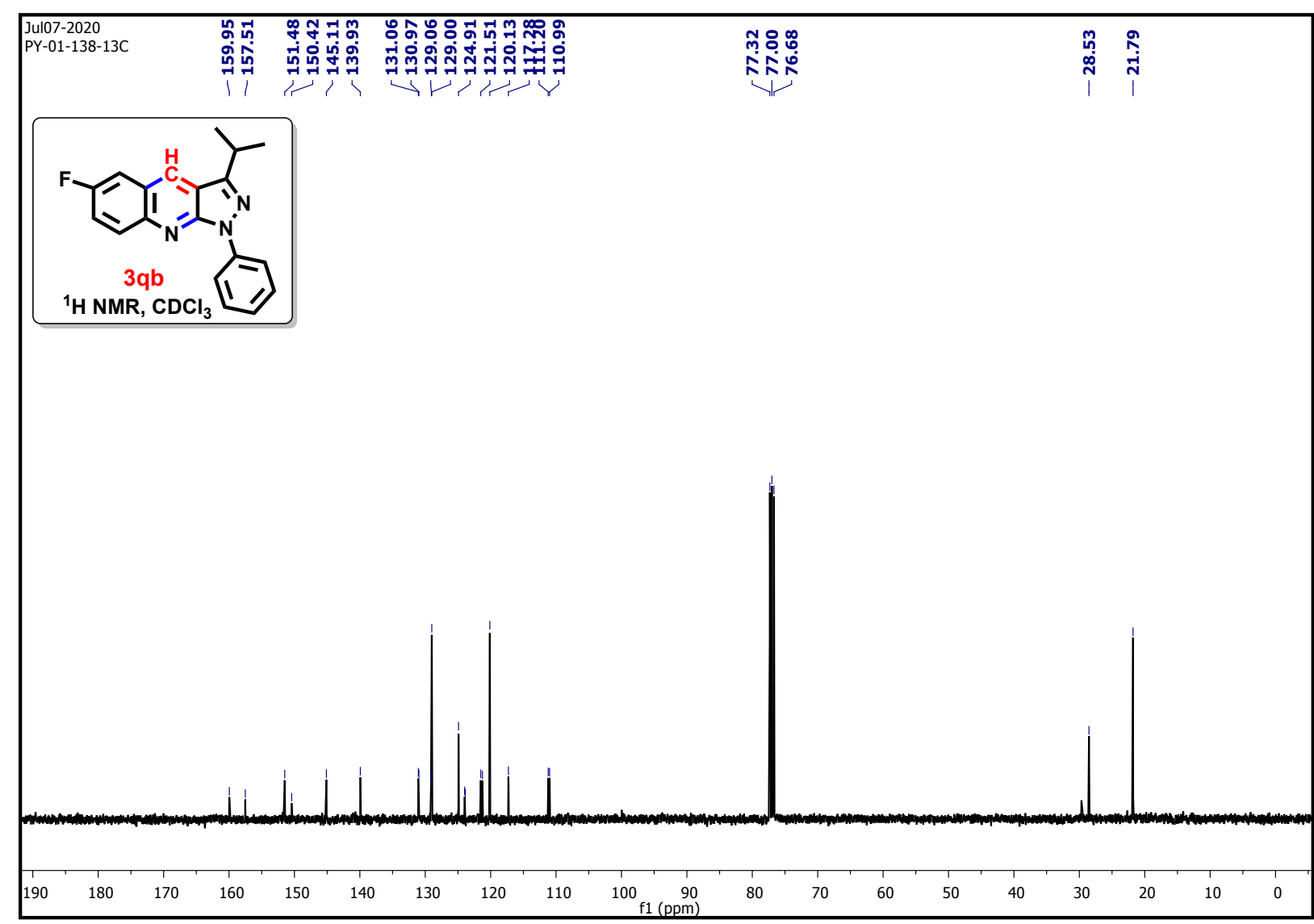


${ }^{1} \mathrm{H}$ NMR (400 MHz) spectra of $3 \mathbf{b c}$

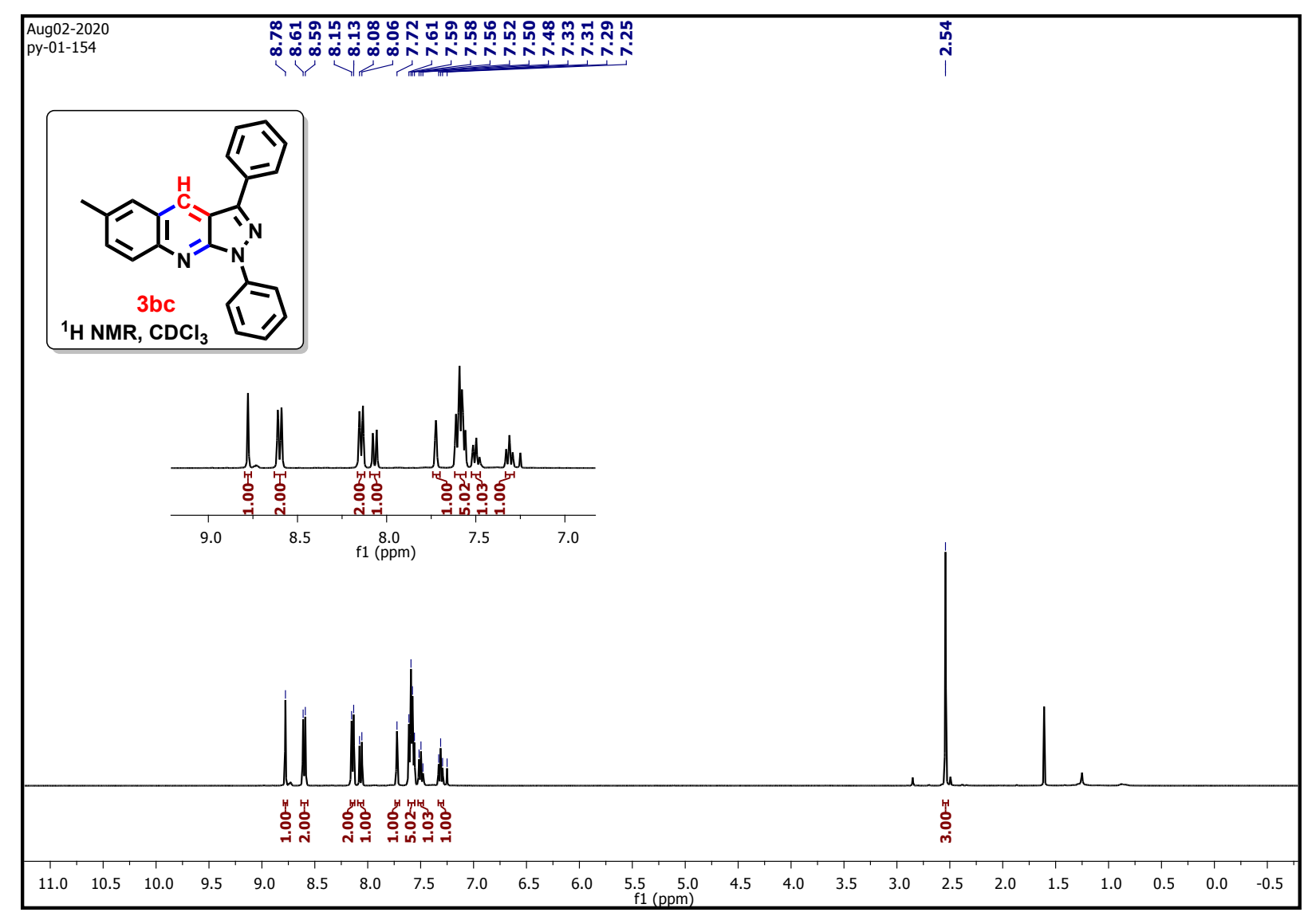

${ }^{13} \mathrm{C}\left\{{ }^{1} \mathrm{H}\right\}$ NMR (101 MHz) spectra of $\mathbf{3 b c}$

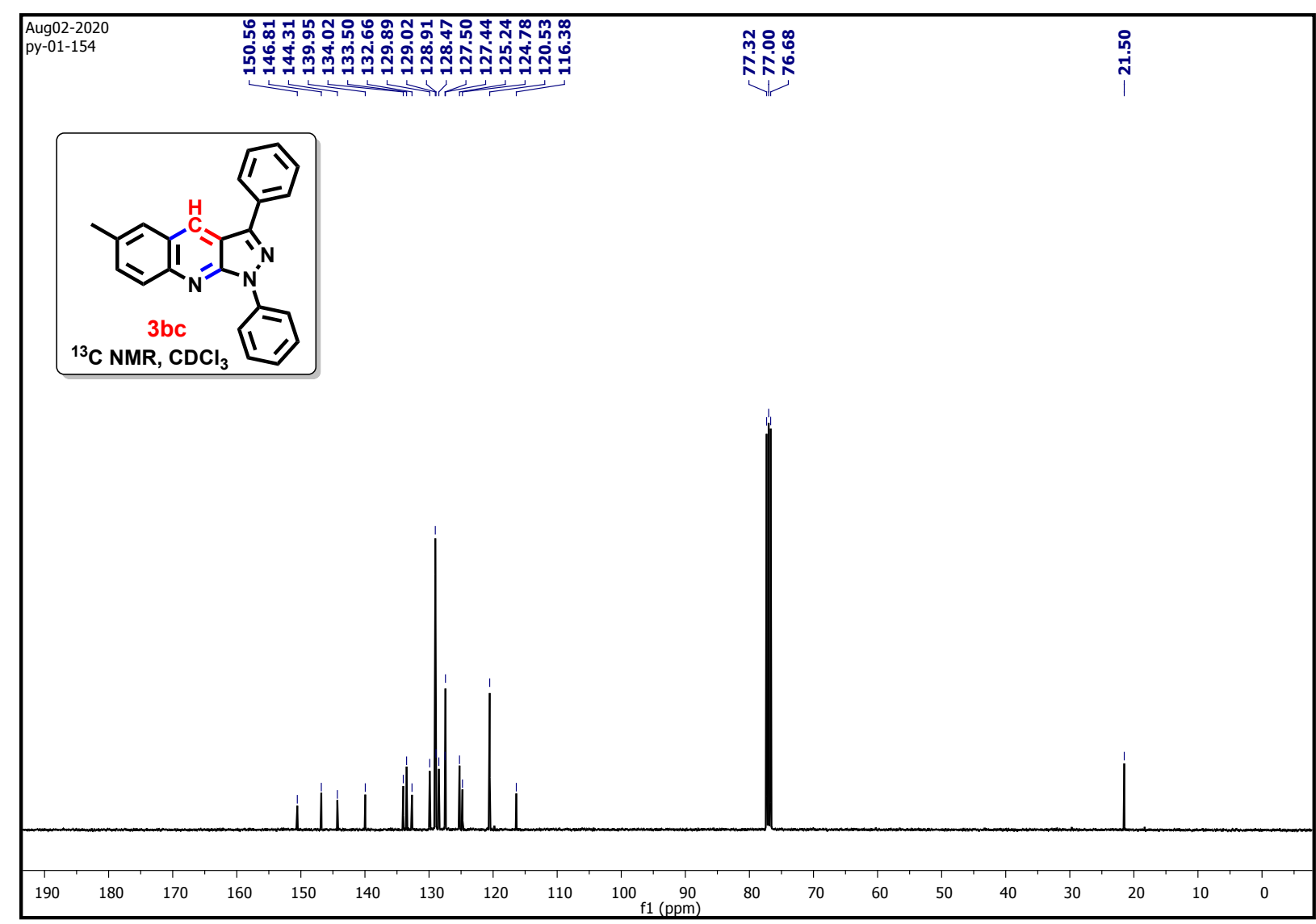


${ }^{1} \mathrm{H}$ NMR (400 MHz) spectra of 3cc

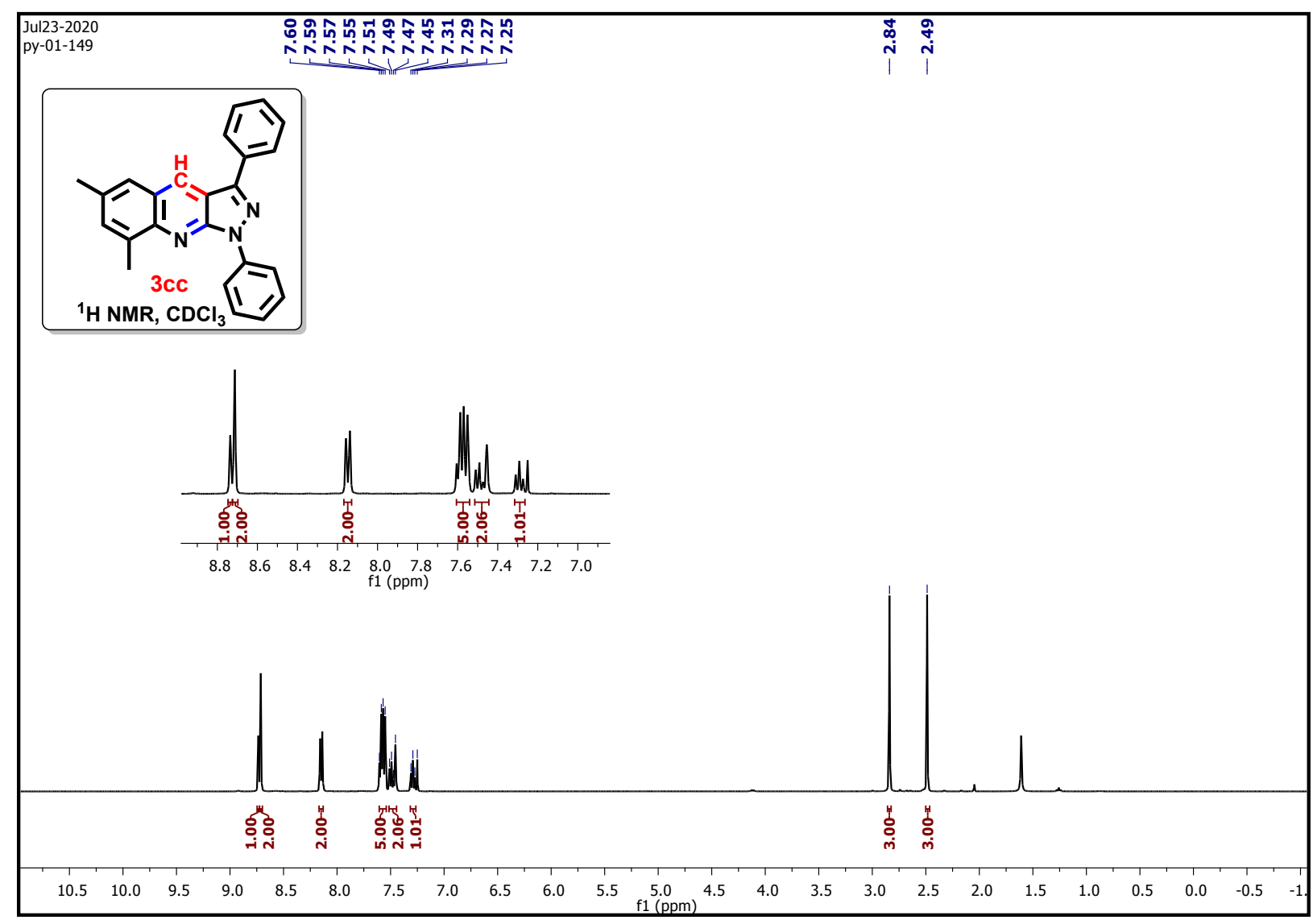

${ }^{13} \mathrm{C}\left\{{ }^{1} \mathrm{H}\right\}$ NMR (101 MHz) spectra of 3cc

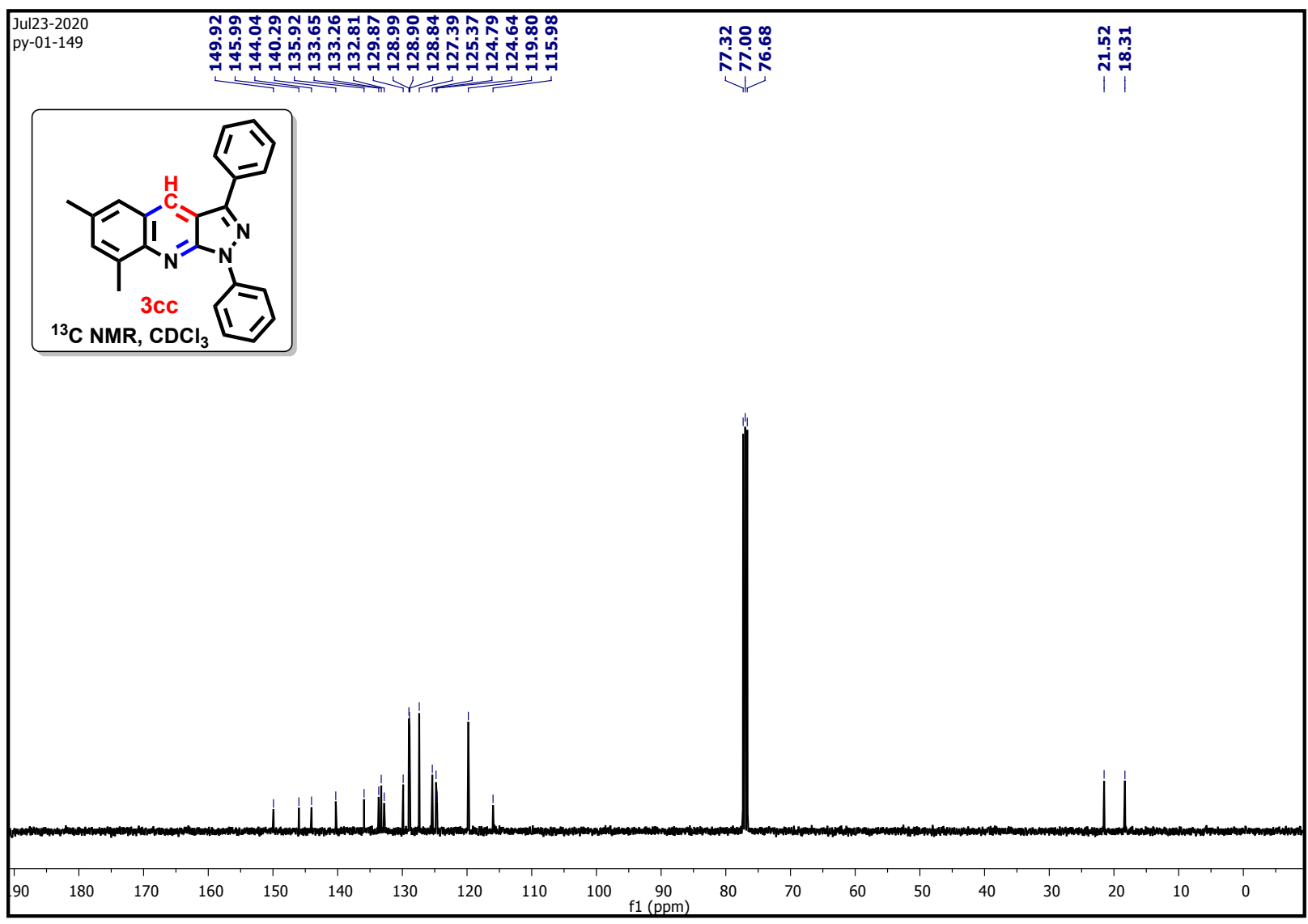


${ }^{1} \mathrm{H}$ NMR $(400 \mathrm{MHz})$ spectra of 3 ic

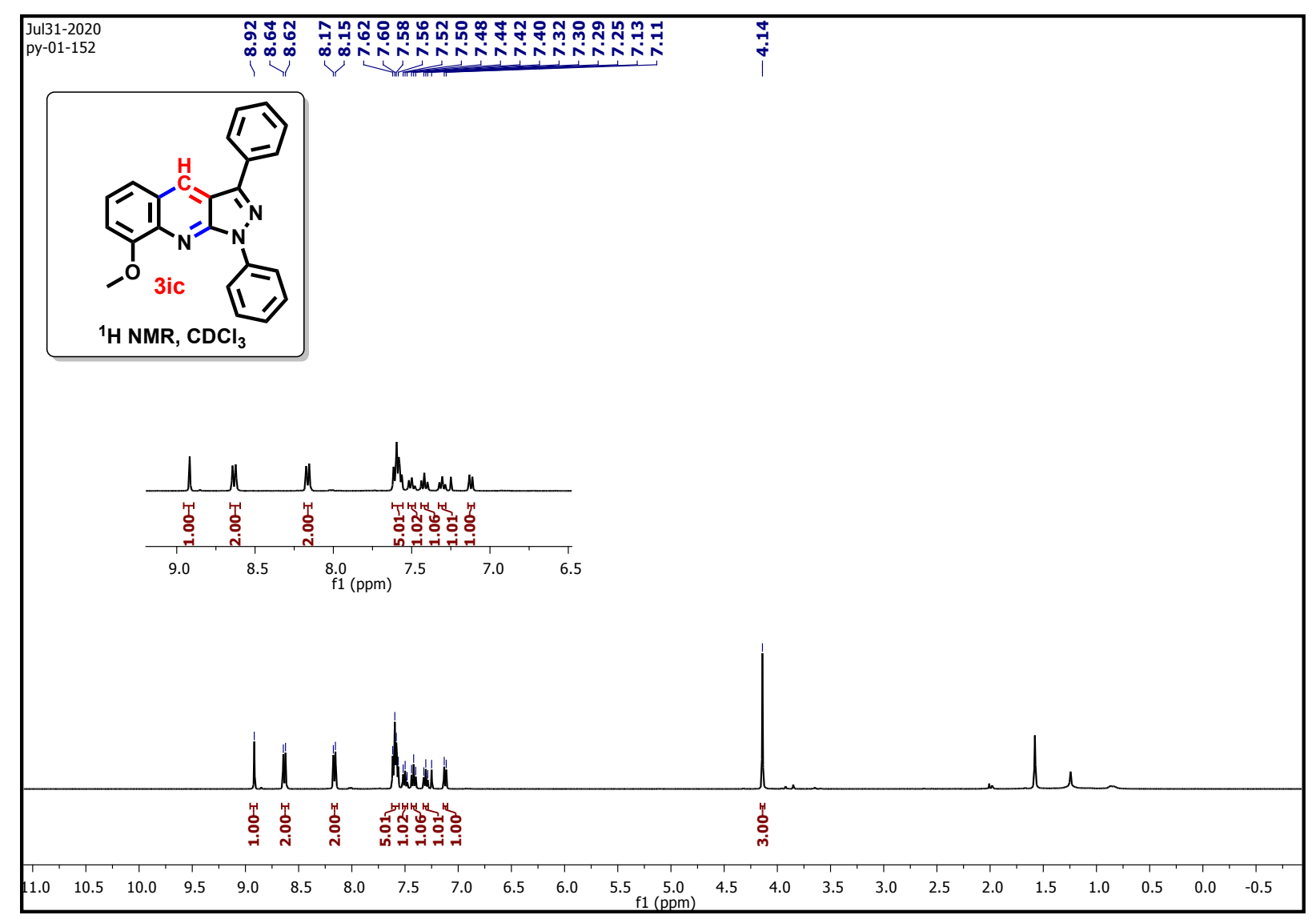

${ }^{13} \mathrm{C}\left\{{ }^{1} \mathrm{H}\right\}$ NMR (101 MHz) spectra of 3ic

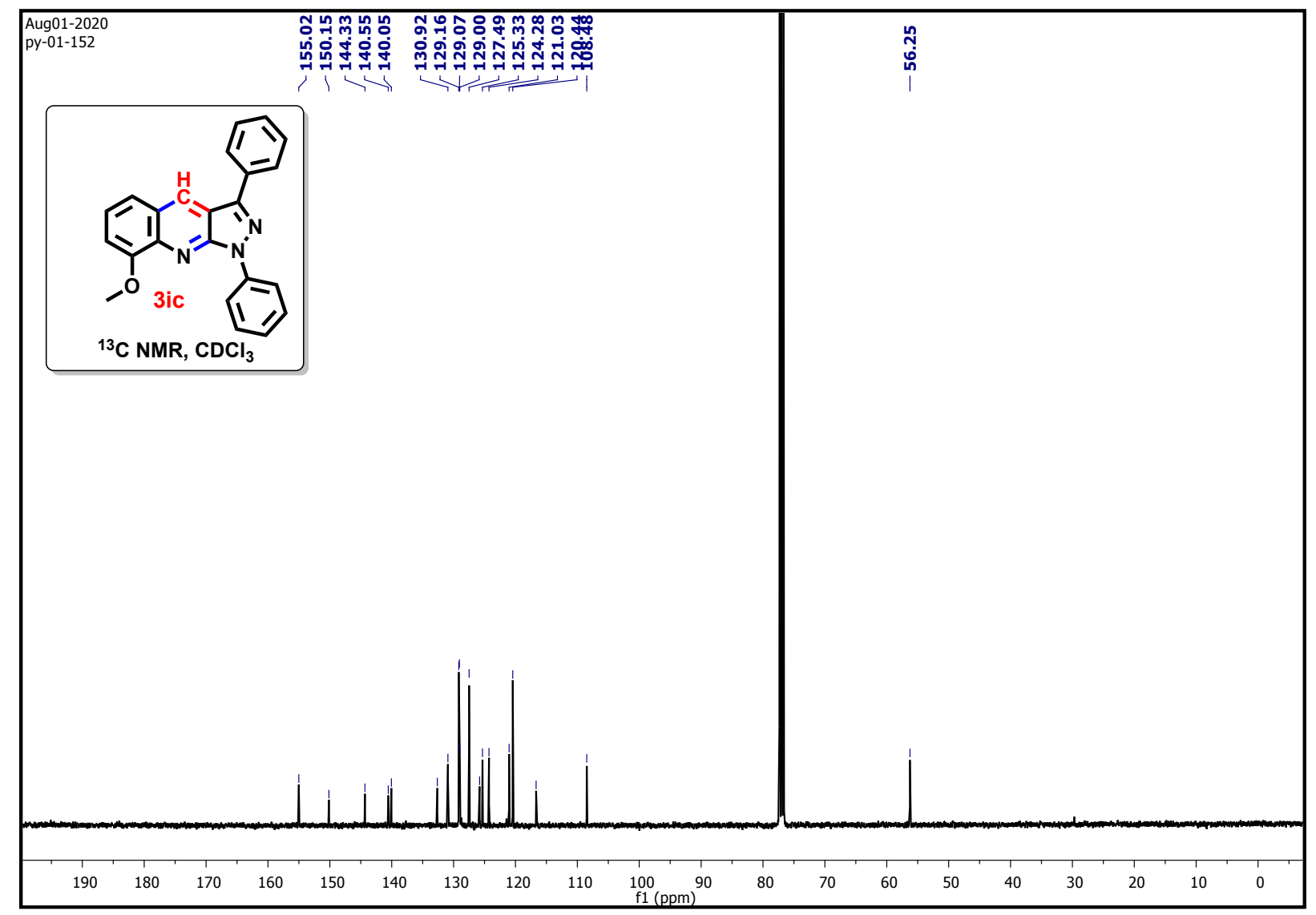


${ }^{1} \mathrm{H}$ NMR (400 MHz) spectra of $30 \mathrm{c}$

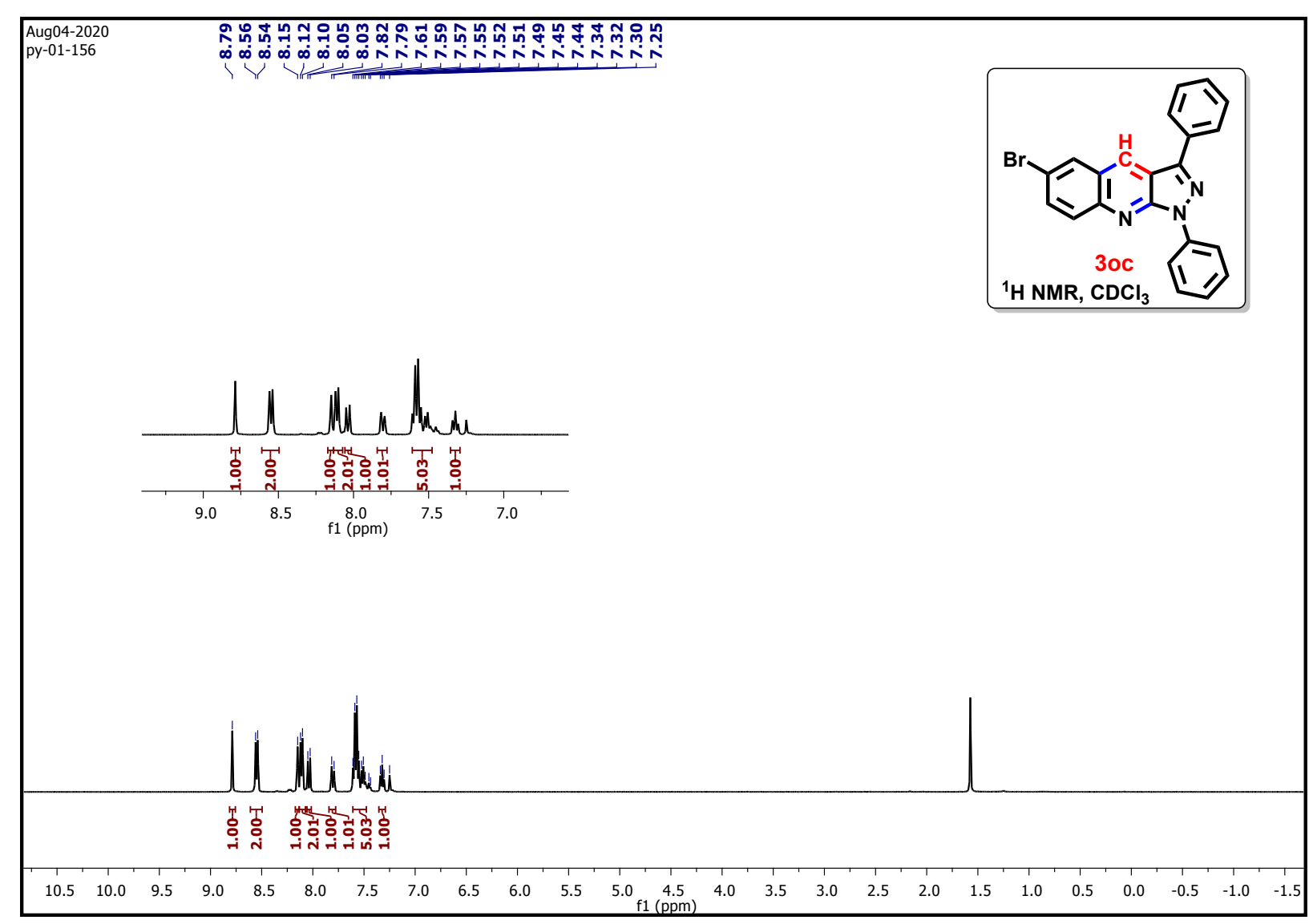

${ }^{13} \mathrm{C}\left\{{ }^{1} \mathrm{H}\right\}$ NMR (101 MHz) spectra of 3oc

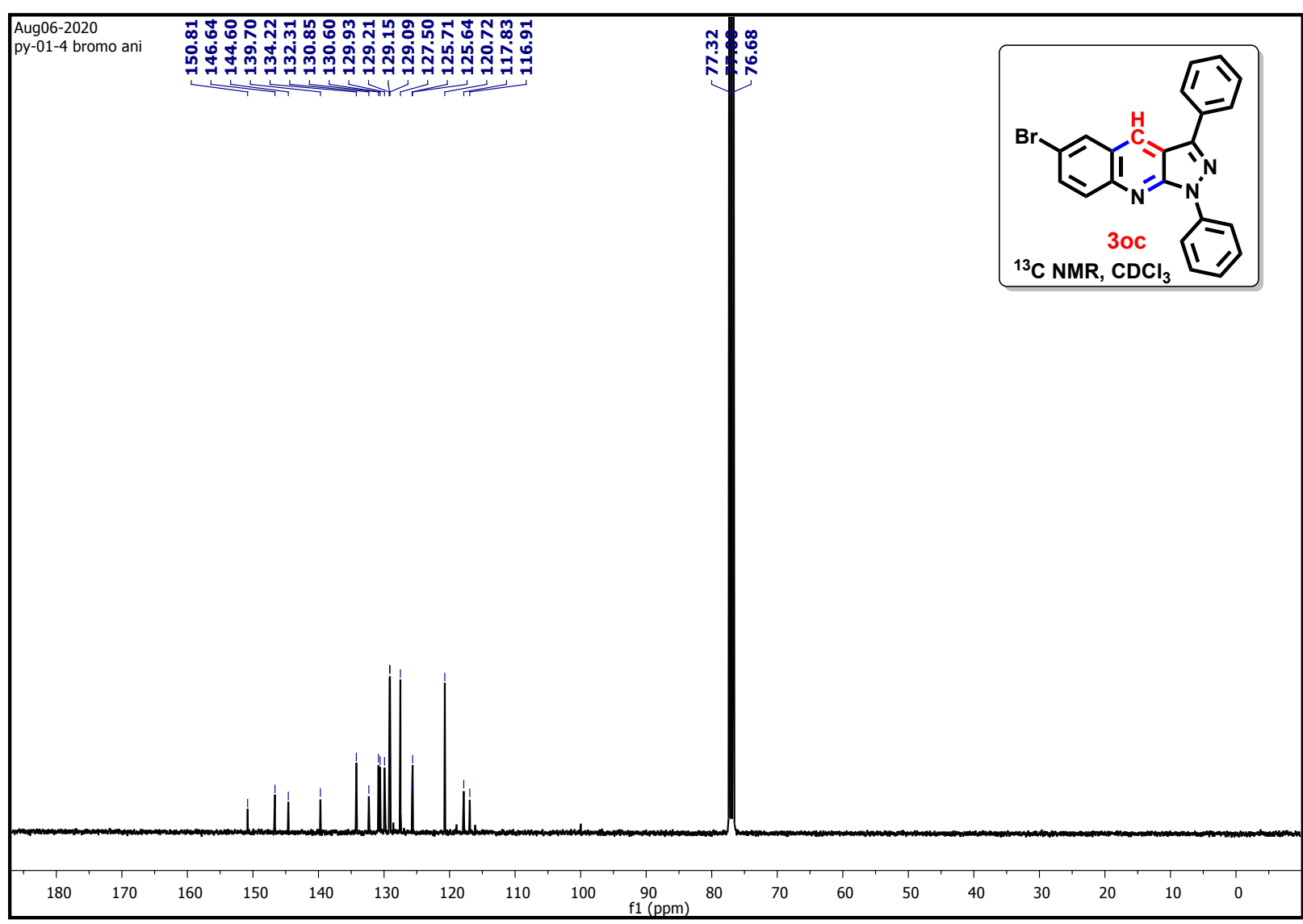


${ }^{1} \mathrm{H}$ NMR (400 MHz) spectra of 3sc

ดें
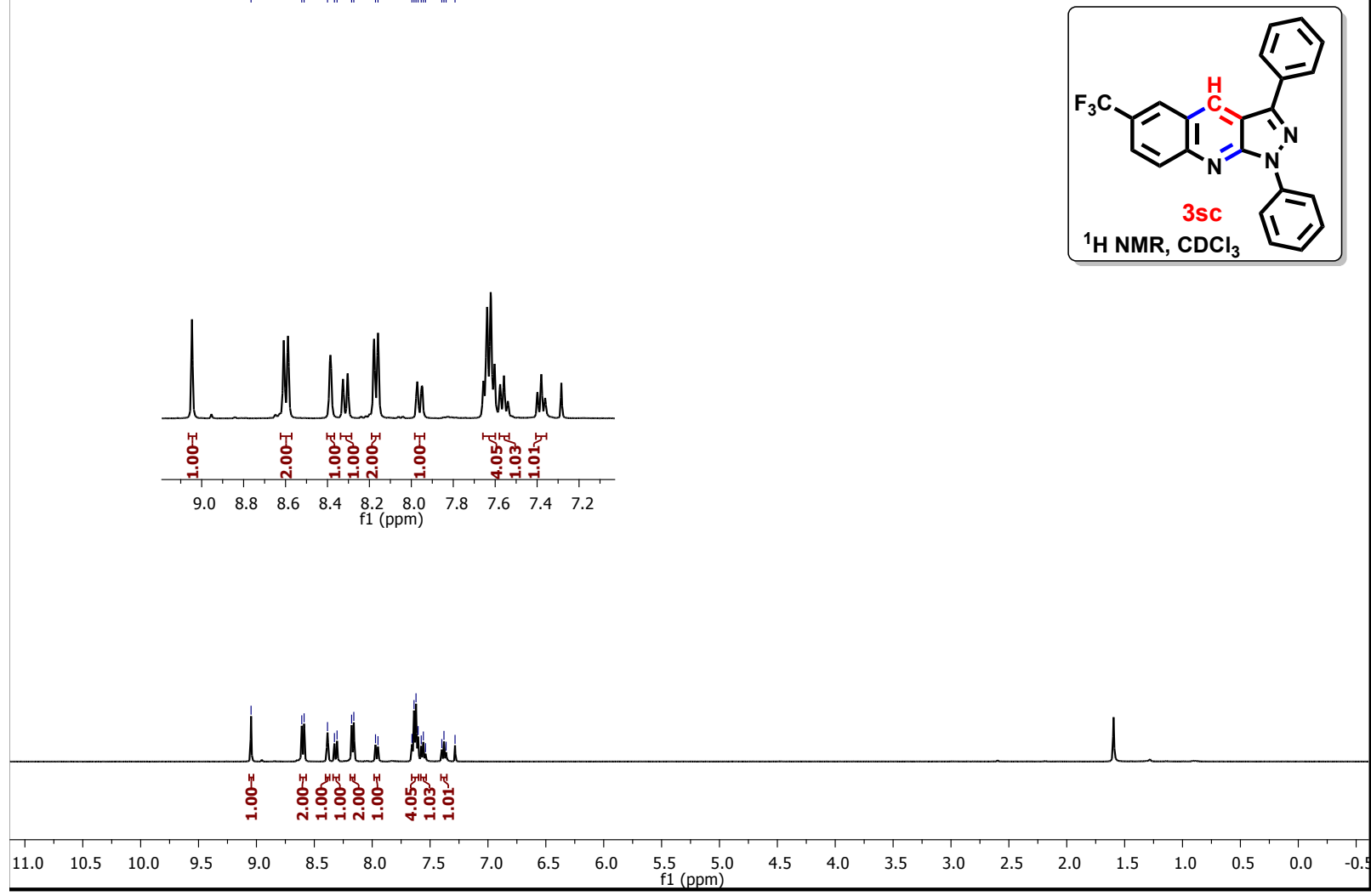

${ }^{13} \mathrm{C}\left\{{ }^{1} \mathrm{H}\right\}$ NMR (101 MHz) spectra of 3sc

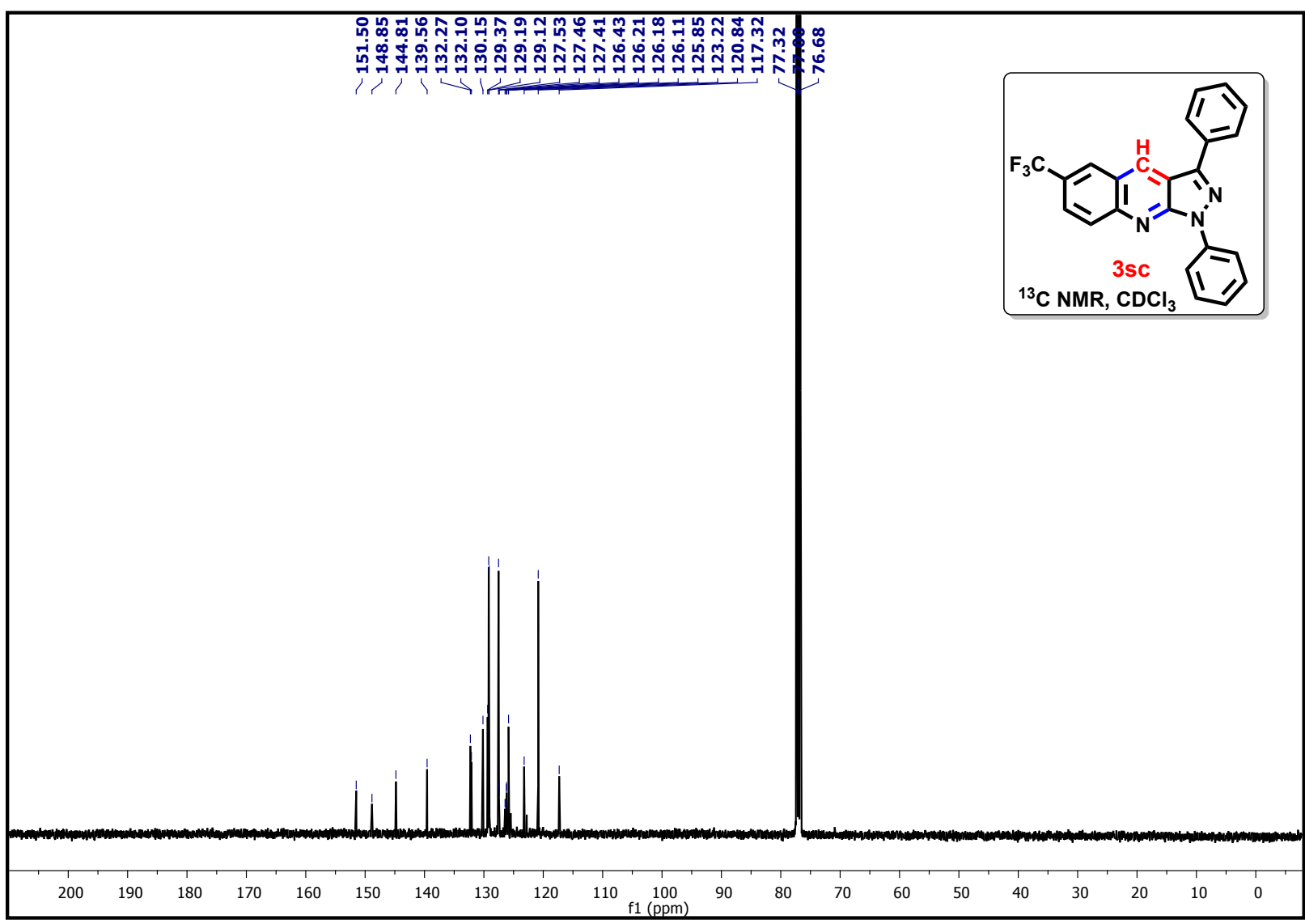


${ }^{1} \mathrm{H}$ NMR (400 MHz) spectra of 3tc

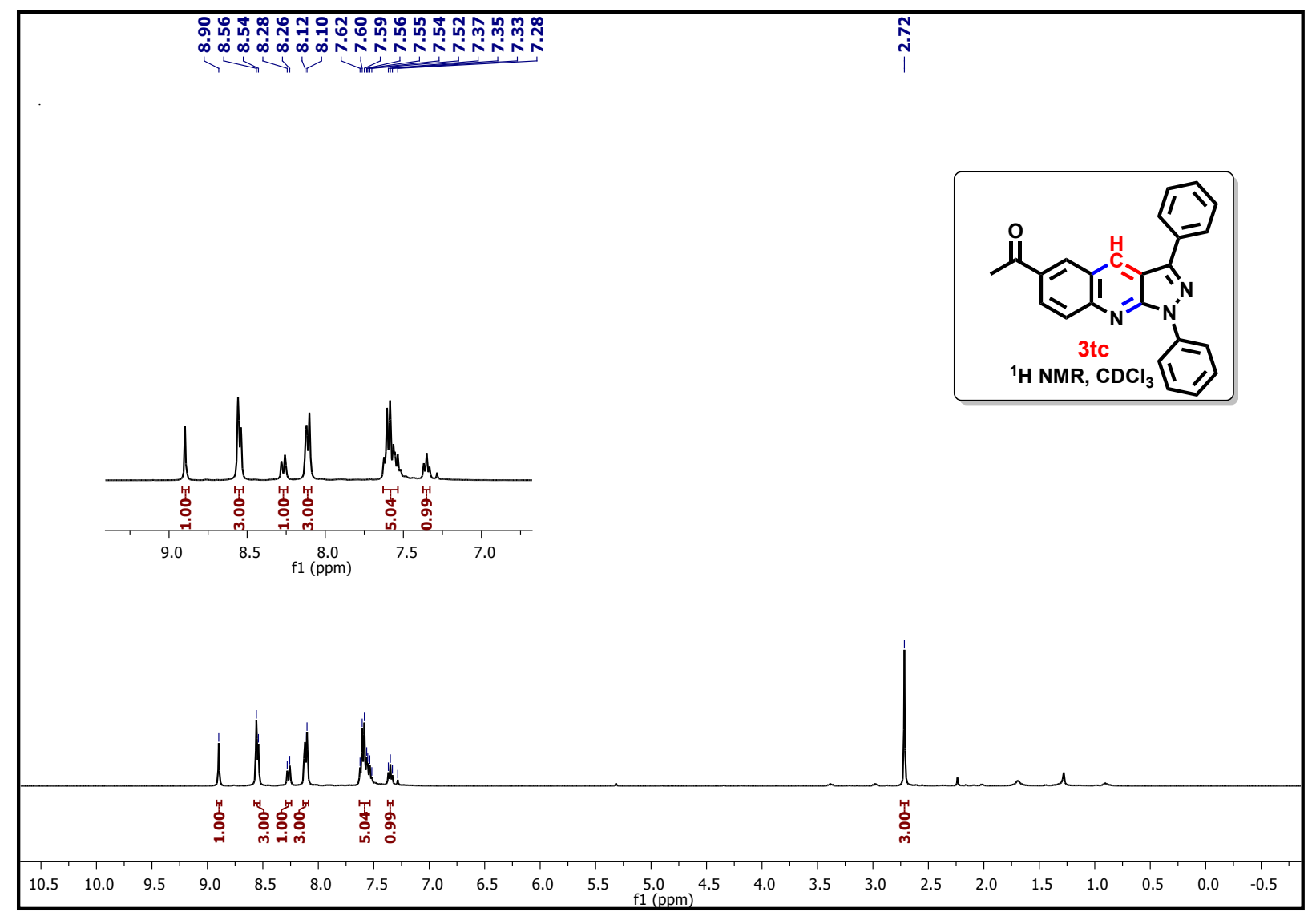

${ }^{13} \mathrm{C}\left\{{ }^{1} \mathrm{H}\right\}$ NMR (101 MHz) spectra of 3tc

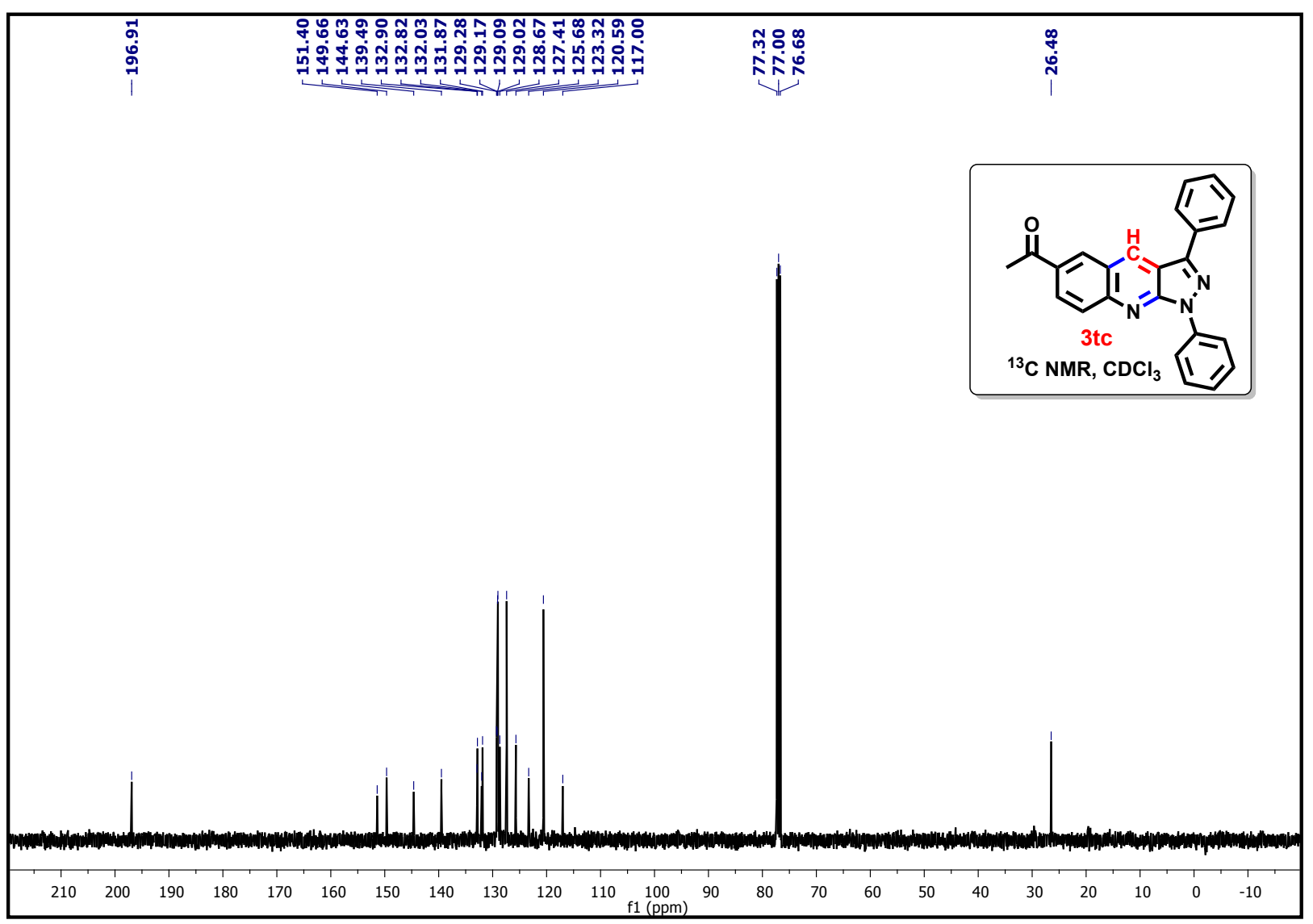


${ }^{1} \mathrm{H} \mathrm{NMR}(400 \mathrm{MHz}$ ) spectra of $\mathbf{3 b d}$

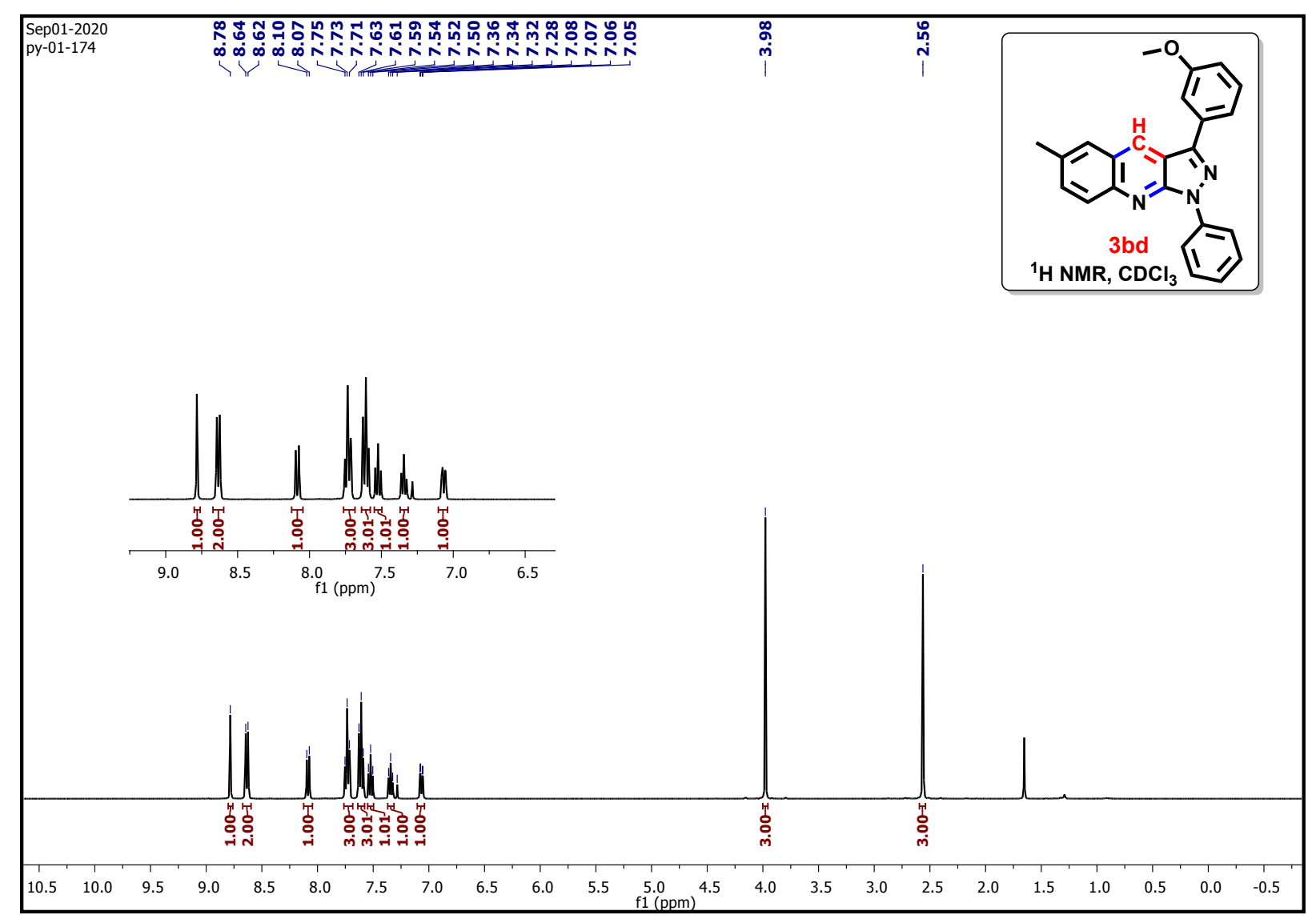

${ }^{13} \mathrm{C}\left\{{ }^{1} \mathrm{H}\right\}$ NMR (101 MHz) spectra of $\mathbf{3 b d}$

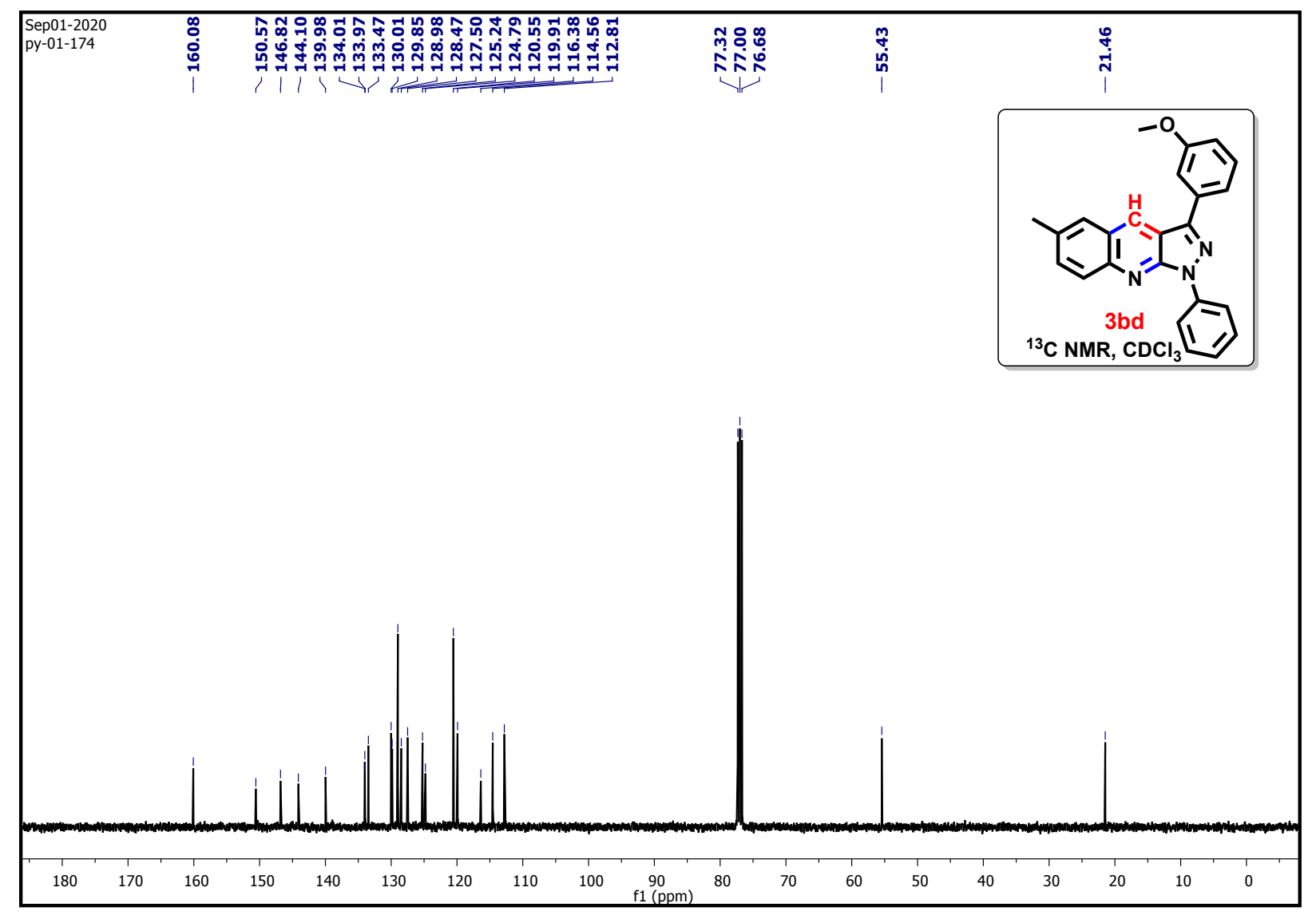


${ }^{1} \mathrm{H}$ NMR (400 MHz) spectra of 4

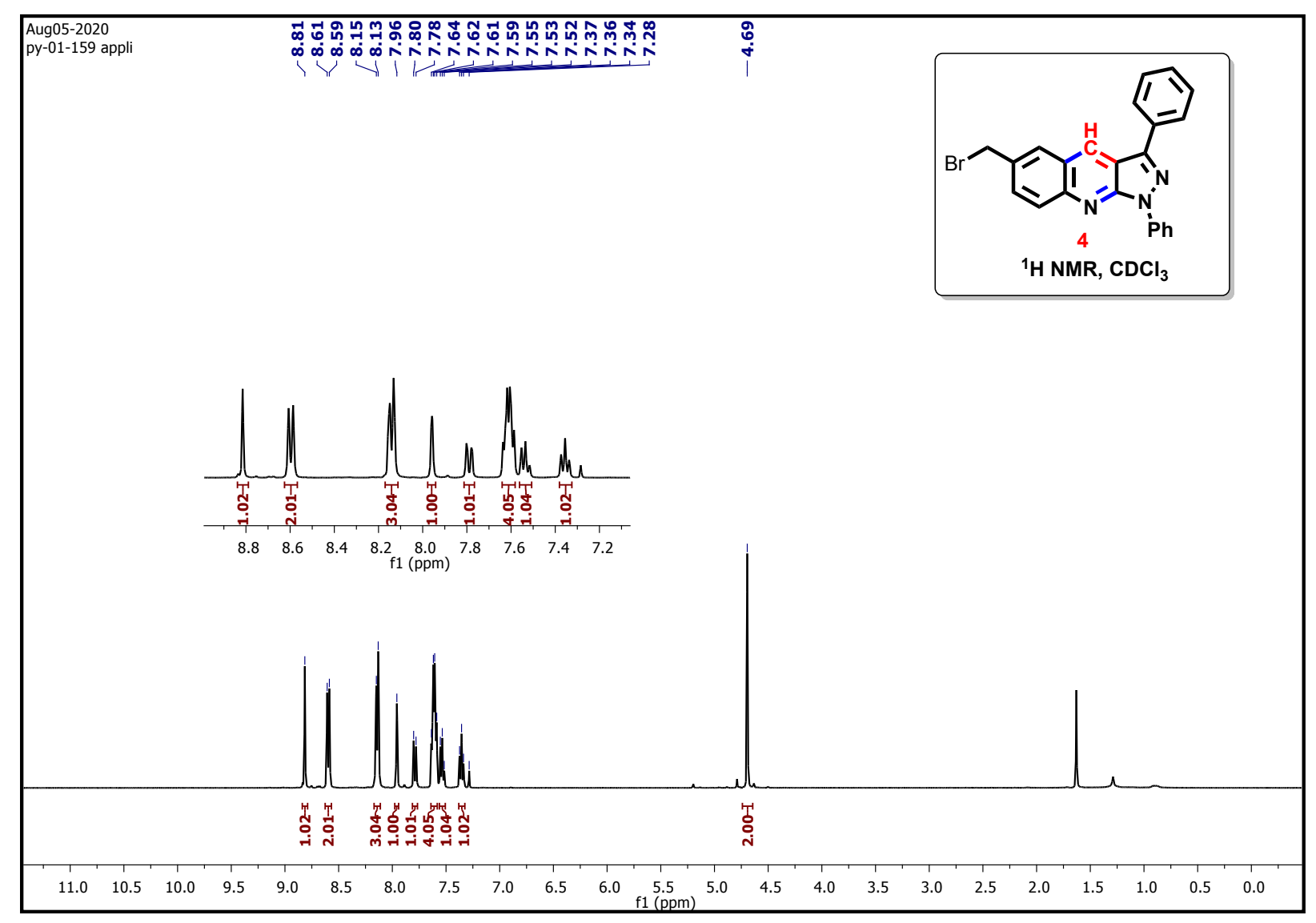

${ }^{13} \mathrm{C}\left\{{ }^{1} \mathrm{H}\right\}$ NMR (101 MHz) spectra of 4

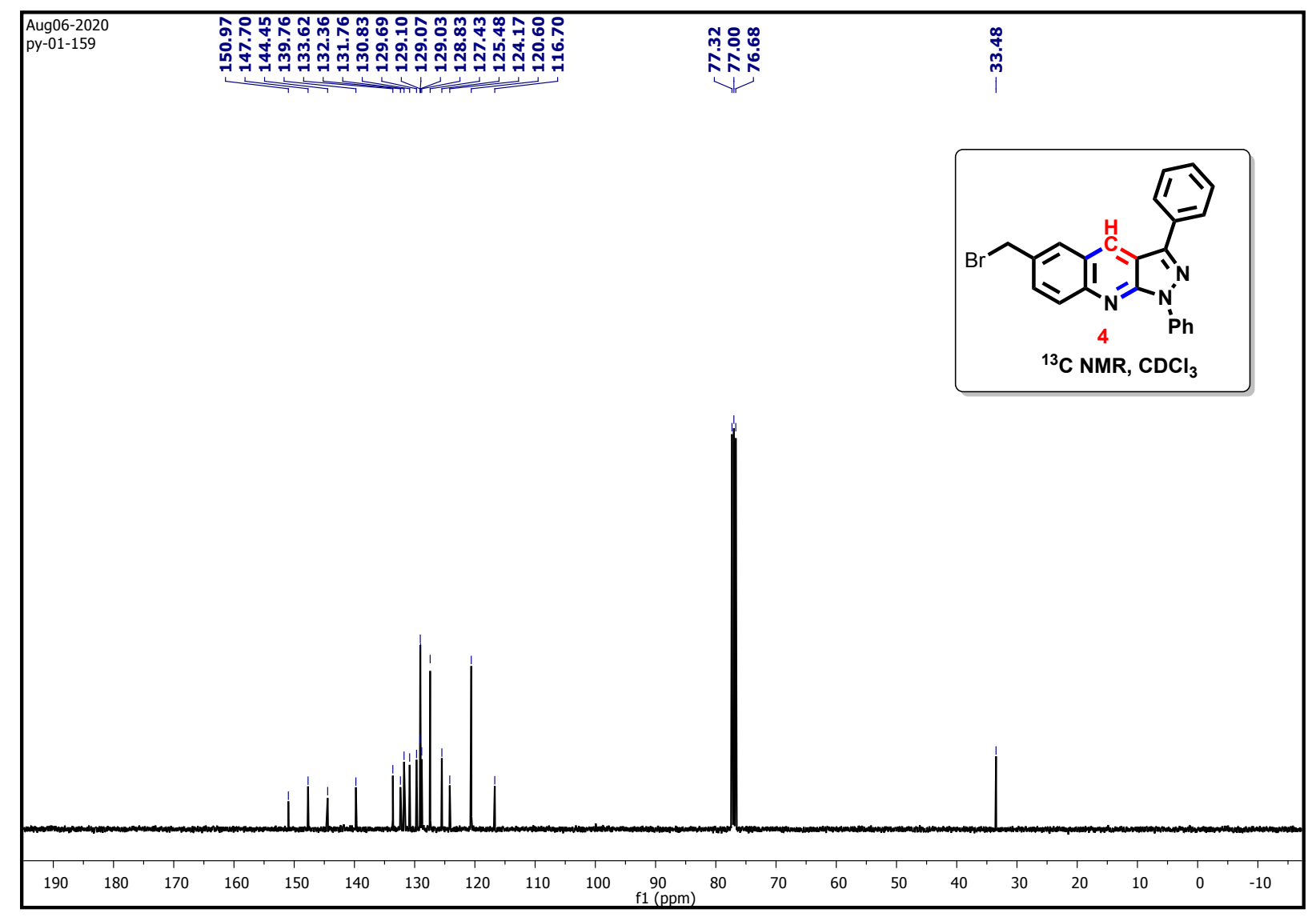


${ }^{1} \mathrm{H}$ NMR (400 MHz) spectra of 3aa-d

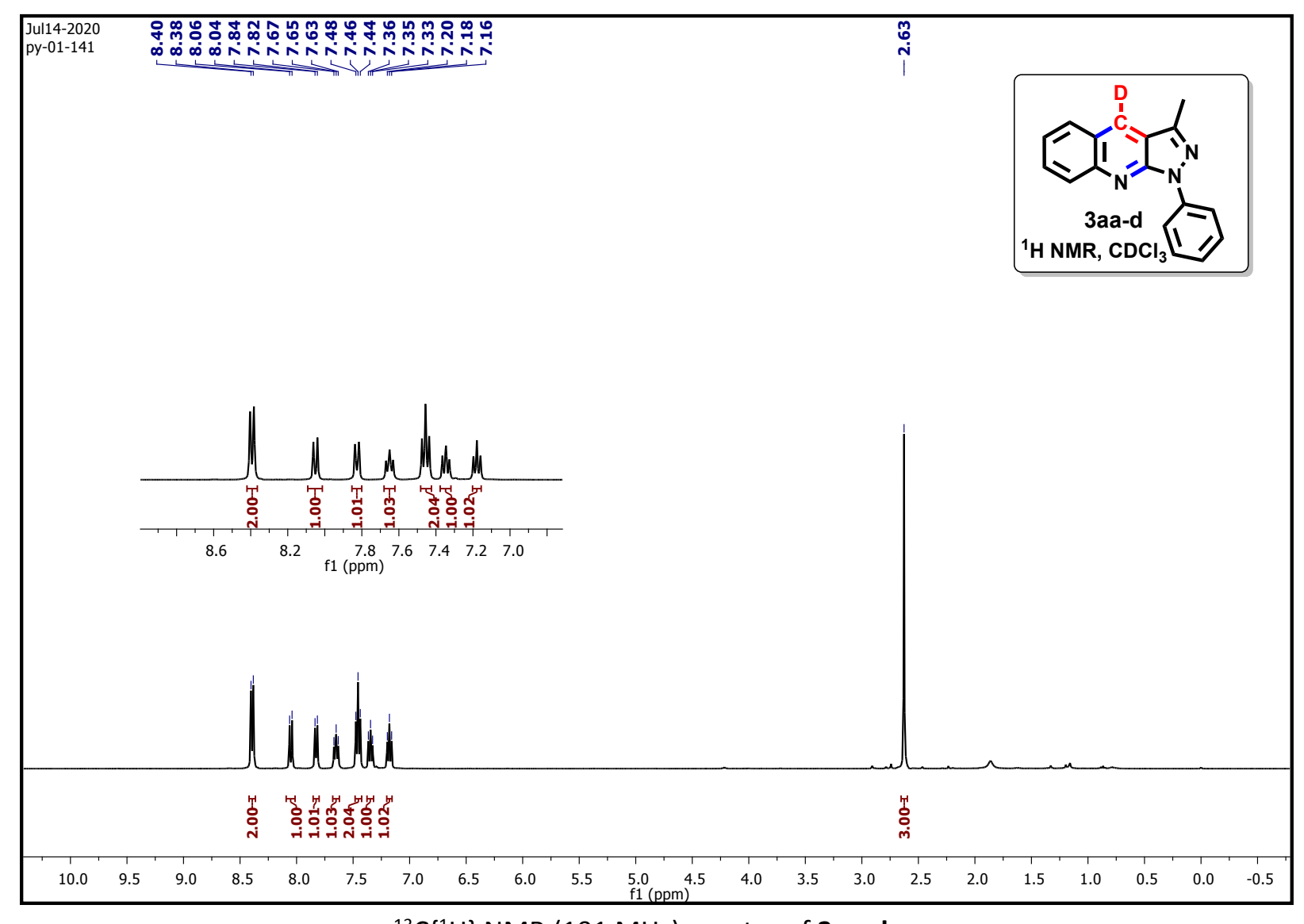

${ }^{13} \mathrm{C}\left\{{ }^{1} \mathrm{H}\right\}$ NMR (101 MHz) spectra of 3aa-d

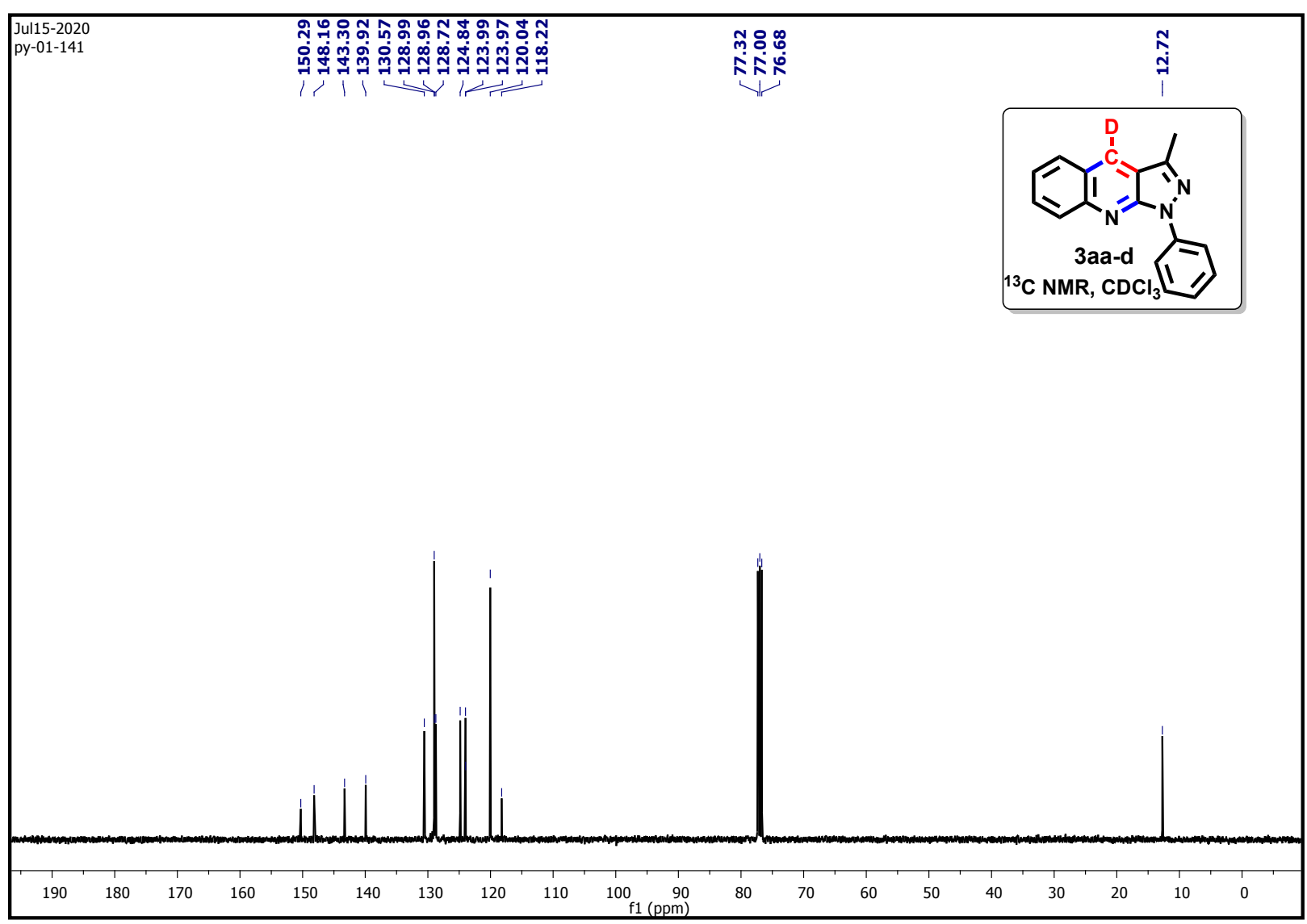




\section{X-Ray Analysis of 3lb (CCDC No. 2032763)}

The single crystals suitable for X-ray diffraction were grown by the vapour diffusion of hexane into dichloromethane solution of the compound 3I $\mathbf{b}$ at room temperature.

Single crystals of each compound 3Ib were mounted under mineral oil on a glass capillary. Data for all compounds were collected at 296 K, on a Bruker Kappa diffractometer equipped with an APEXII CCD detector and Mo fine focus sealed tube source. All the data were collected by employing graphite $\mathrm{S} 3$ monochromated $\mathrm{M}_{0} K_{\alpha}$ radiation $(\lambda=0.7107 \AA)$ and the data sets were processed using APEX II software. Integration of the data sets were carried out with the Bruker SAINT program. Structure solutions were performed using the SHELXTL/PC suite. ${ }^{\mathrm{S} 1}$ Intensities were corrected for Lorentz and polarization effects and an empirical absorption correction was applied using Blessing's method as incorporated into the program SADABS. ${ }^{\mathrm{S} 2}$ Non-hydrogen atoms were refined with anisotropic thermal parameters and hydrogen atoms were included in idealized positions.

(S1) (a) SHELXTL-PC, Vers. 5.10; 1998, Bruker-Analytical X-ray Services, Madison, WI.

(b) G. M. Sheldrick, SHELX-97, Universität Göttingen, Göttingen, Germany. (S2) SADABS; G. M. Sheldrick, 1996, based on the method described in R. H. Blessing, Acta Crystallogr. Sect. A, 1995, 51, 33-38. 


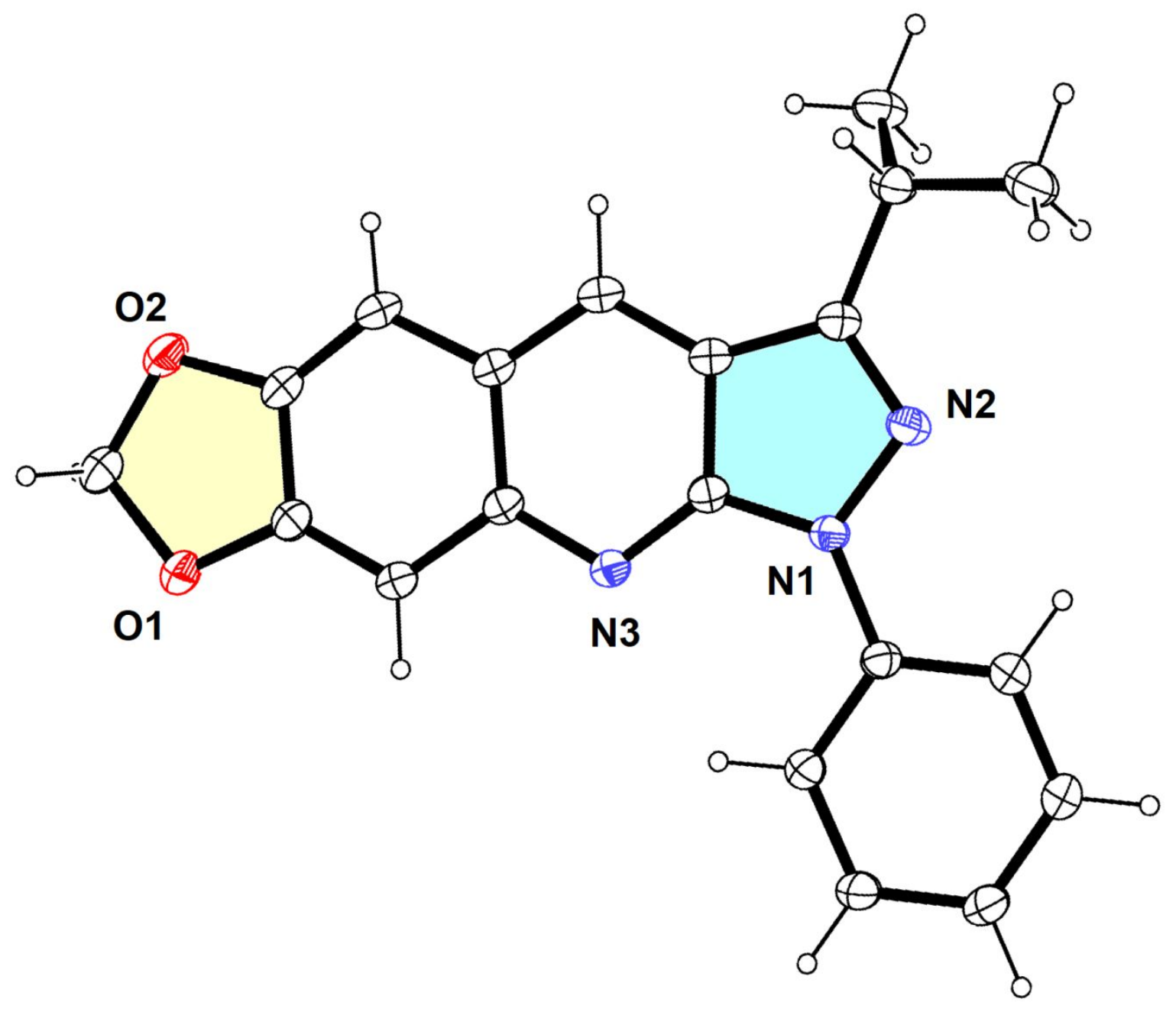

Figure S1 X-ray structure of 3lb; The thermal ellipsoid plots are drawn at 30\% probability level.

\section{Table S1}

Identification code

Empirical formula

Formula weight

Temperature/K

Crystal system

Space group
Dharmendar 31b

$\mathrm{C}_{20} \mathrm{H}_{17} \mathrm{~N}_{3} \mathrm{O}_{2}$

331.36

296(2)

Triclinic

P-1 


\begin{tabular}{|c|c|}
\hline $\mathrm{a} / \AA$ & $8.5093(16)$ \\
\hline $\mathrm{b} / \AA \AA$ & $9.8568(19)$ \\
\hline $\mathrm{c} / \AA$ & $11.112(2)$ \\
\hline$\alpha^{\circ}$ & $86.750(6)$ \\
\hline$\beta /{ }^{\circ}$ & $74.003(6)$ \\
\hline$\gamma /{ }^{\circ}$ & $64.768(6)$ \\
\hline Volume $/ \AA^{3}$ & $808.4(3)$ \\
\hline $\mathrm{Z}$ & 2 \\
\hline$\rho_{\text {calc }} \mathrm{g} / \mathrm{cm}^{3}$ & 1.361 \\
\hline$\mu / \mathrm{mm}^{-1}$ & 0.090 \\
\hline $\mathrm{F}(000)$ & 348.0 \\
\hline Crystal size $/ \mathrm{mm}^{3}$ & $0.170 \times 0.095 \times 0.045$ \\
\hline Radiation & $\operatorname{MoK} \alpha(\lambda=0.71073)$ \\
\hline $2 \Theta$ range for data collection $/^{\circ}$ & 4.58 to 49.99 \\
\hline Index ranges & $-10 \leq \mathrm{h} \leq 10,-11 \leq \mathrm{k} \leq 11,-13 \leq 1 \leq 13$ \\
\hline Reflections collected & 17546 \\
\hline Independent reflections & $2837\left[\mathrm{R}_{\mathrm{int}}=0.0433, \mathrm{R}_{\mathrm{sigma}}=0.0325\right]$ \\
\hline Data/restraints/parameters & $2837 / 0 / 229$ \\
\hline Goodness-of-fit on $\mathrm{F}^{2}$ & 1.044 \\
\hline Final $R$ indexes $[I>=2 \sigma(I)]$ & $\mathrm{R}_{1}=0.0433, \mathrm{wR}_{2}=0.0989$ \\
\hline Final $R$ indexes [all data] & $\mathrm{R}_{1}=0.0732, \mathrm{wR}_{2}=0.1224$ \\
\hline Largest diff. peak/hole / e $\AA^{-3}$ & $0.19 /-0.13$ \\
\hline
\end{tabular}

Table S2 Fractional Atomic Coordinates $\left(\times 10^{4}\right)$ and Equivalent Isotropic Displacement Parameters $\left(\AA^{2} \times 10^{3}\right)$ for Dharmendar 31b. $U_{\text {eq }}$ is defined as $1 / 3$ of of the trace of the orthogonalised $\mathrm{U}_{\mathrm{IJ}}$ tensor.

$\begin{array}{lcc}\text { Atom } & \boldsymbol{x} & \boldsymbol{y} \\ \text { C1 } & 1034(3) & 3429(3) \\ \text { C2 } & 1859(3) & 3466(2) \\ \text { C3 } & 1167(3) & 4933(2) \\ \text { C4 } & 1073(3) & 6095(2) \\ \text { C5 } & 1716(2) & 5801(2) \\ \text { C6 } & 2443(2) & 4291(2) \\ \text { C7 } & 2495(3) & 3112(2) \\ \text { C8 } & 1638(3) & 6940(2) \\ \text { C9 } & 2252(3) & 6578(2) \\ \text { C10 } & 2956(2) & 5053(2) \\ \text { C11 } & 2354(3) & 7360(2) \\ \text { C12 } & 1699(3) & 9030(2) \\ \text { C13 } & 2738(3) & 9631(2) \\ \text { C14 } & 1743(4) & 9452(3) \\ \text { C15 } & 4289(3) & 3738(2) \\ \text { C16 } & 4937(3) & 3926(2) \\ \text { C17 } & 5771(4) & 2718(3) \\ \text { C18 } & 5984(3) & 1315(3) \\ \text { C19 } & 5333(3) & 1131(2)\end{array}$

$z$ $\mathbf{U}(\mathbf{e q})$

$838(2) 58.4(6)$

$2576.7(18) 45.4(5)$

$2199.9(19) 48.6(5)$

$2836(2) 52.6(6)$

$3922.8(18) 42.6(5)$

$4297.9(17) 41.1(5)$

$3588.0(18) 47.1(5)$

$4657.3(19) 47.8(5)$

$5705.4(18) 42.9(5)$

$5971.9(17) 40.1(5)$

$6706.5(19) 45.8(5)$

$6922(2) 54.9(6)$

$5866(2) 67.8(7)$

$8188(2) 87.6(9)$

$7723.7(17) 42.3(5)$

$8672(2) 57.9(6)$

$9318(2) 67.2(7)$

$9014(2) 62.2(6)$

$8073(2) 65.7(7)$ 


$\begin{array}{lrr}\text { C20 } & 4472(3) & 2330(2) \\ \text { N1 } & 3461(2) & 4984.5(17) \\ \text { N2 } & 3072(2) & 6415.8(18) \\ \text { N3 } & 3076(2) & 3913.1(17) \\ \text { O1 } & 1747(2) & 2527.8(17) \\ \text { O2 } & 620(2) & 4945.7(18)\end{array}$

$7429(2) 58.1(6)$

$7055.6(15) 43.8(4)$

$7491.9(15) 47.7(4)$

$5320.6(15) 43.5(4)$

$1781.7(14) 62.5(4)$

$1152.9(14) 67.5(5)$

Table S3 Anisotropic Displacement Parameters $\left(\AA^{2} \times 10^{3}\right)$ for Dharmendar 31b. The Anisotropic displacement factor exponent takes the form: -

\section{$2 \pi^{2}\left[h^{2} a * 2 U_{11}+2 h k a * b * U_{12}+\ldots\right]$.}

\begin{tabular}{lrrrrrr} 
Atom & \multicolumn{1}{c}{$\mathbf{U}_{11}$} & $\mathbf{U}_{\mathbf{2 2}}$ & $\mathbf{U}_{\mathbf{3 3}}$ & \multicolumn{1}{c}{$\mathbf{U}_{\mathbf{2 3}}$} & \multicolumn{1}{c}{$\mathbf{U}_{\mathbf{1 3}}$} & \multicolumn{1}{c}{$\mathbf{U}_{\mathbf{1 2}}$} \\
$\mathrm{C} 1$ & $60.7(14)$ & $65.0(16)$ & $48.6(13)$ & $10.3(11)$ & $-22.2(11)$ & $-22.6(12)$ \\
C2 & $44.5(12)$ & $48.4(12)$ & $41.1(11)$ & $5.7(9)$ & $-12.5(9)$ & $-17.9(10)$ \\
C3 & $44.9(12)$ & $53.3(14)$ & $42.6(12)$ & $10.6(10)$ & $-16.0(10)$ & $-15.1(10)$ \\
C4 & $52.5(13)$ & $44.3(13)$ & $53.3(13)$ & $15.1(10)$ & $-20.1(10)$ & $-11.9(10)$ \\
C5 & $37.6(11)$ & $42.2(12)$ & $42.4(11)$ & $8.4(9)$ & $-9.2(9)$ & $-13.6(9)$ \\
C6 & $36.4(11)$ & $43.3(12)$ & $40.3(11)$ & $8.2(9)$ & $-8.7(9)$ & $-15.9(9)$ \\
C7 & $51.7(13)$ & $41.0(12)$ & $48.3(12)$ & $10.4(9)$ & $-17.7(10)$ & $-18.1(10)$ \\
C8 & $45.6(12)$ & $37.4(11)$ & $53.0(13)$ & $10.2(10)$ & $-12.1(10)$ & $-12.9(9)$ \\
C9 & $40.1(11)$ & $37.6(11)$ & $45.8(12)$ & $6.7(9)$ & $-8.4(9)$ & $-14.5(9)$ \\
C10 & $38.2(11)$ & $39.5(11)$ & $39.1(11)$ & $5.2(9)$ & $-7.1(9)$ & $-15.8(9)$ \\
C11 & $47.2(12)$ & $39.0(11)$ & $47.0(12)$ & $6.0(10)$ & $-9.1(10)$ & $-17.2(10)$ \\
C12 & $63.0(14)$ & $37.3(12)$ & $57.4(14)$ & $2.1(10)$ & $-16.5(11)$ & $-14.9(10)$ \\
C13 & $81.4(17)$ & $42.9(13)$ & $75.4(17)$ & $12.5(12)$ & $-17.9(14)$ & $-26.6(13)$ \\
C14 & $141(3)$ & $48.5(15)$ & $69.1(18)$ & $3.5(13)$ & $-34.8(18)$ & $-33.4(17)$ \\
C15 & $46.0(12)$ & $38.3(11)$ & $39.7(11)$ & $5.8(9)$ & $-10.0(9)$ & $-16.8(9)$ \\
C16 & $75.2(16)$ & $44.6(13)$ & $62.5(14)$ & $6.1(11)$ & $-32.7(12)$ & $-25.6(12)$ \\
C17 & $90.4(18)$ & $56.4(15)$ & $68.6(16)$ & $11.7(12)$ & $-46.9(14)$ & $-29.9(13)$ \\
C18 & $79.6(17)$ & $47.5(14)$ & $58.8(15)$ & $14.7(11)$ & $-33.5(13)$ & $-19.2(12)$ \\
C19 & $102.3(19)$ & $37.4(13)$ & $58.7(14)$ & $7.4(10)$ & $-34.7(14)$ & $-24.0(13)$ \\
C20 & $88.3(17)$ & $42.6(13)$ & $48.7(13)$ & $7.1(10)$ & $-30.8(12)$ & $-26.2(12)$ \\
N1 & $53.7(10)$ & $34.1(9)$ & $43.2(10)$ & $5.7(7)$ & $-15.3(8)$ & $-17.8(8)$ \\
N2 & $55.0(11)$ & $36.7(10)$ & $48.1(10)$ & $2.1(8)$ & $-9.9(8)$ & $-19.1(8)$ \\
N3 & $47.3(10)$ & $39.5(10)$ & $43.5(10)$ & $8.1(8)$ & $-15.2(8)$ & $-17.6(8)$ \\
O1 & $83.7(11)$ & $57.2(10)$ & $55.0(9)$ & $10.8(8)$ & $-37.8(8)$ & $-27.5(9)$ \\
O2 & $86.4(12)$ & $57.2(10)$ & $58.6(10)$ & $11.9(8)$ & $-40.9(9)$ & $-18.9(9)$
\end{tabular}

Table S4 Bond Lengths for Dharmendar $31 \mathrm{~b}$.

\begin{tabular}{|c|c|c|c|c|c|}
\hline At & Atom & Length/Å & Ator & Atom & Length/Å \\
\hline $\mathrm{C} 1$ & $\mathrm{O} 2$ & $1.422(3)$ & $\mathrm{C} 10$ & N3 & $1.323(2)$ \\
\hline $\mathrm{C} 1$ & $\mathrm{O} 1$ & $1.429(2)$ & $\mathrm{C} 10$ & N1 & $1.375(2)$ \\
\hline $\mathrm{C} 2$ & $\mathrm{C} 7$ & $1.344(3)$ & C11 & $\mathrm{N} 2$ & $1.311(2)$ \\
\hline
\end{tabular}




$\begin{array}{llllll}\text { C2 } & \text { O1 } & 1.363(2) & \mathrm{C} 11 & \mathrm{C} 12 & 1.506(3) \\ \mathrm{C} 2 & \mathrm{C} 3 & 1.398(3) & \mathrm{C} 12 & \mathrm{C} 14 & 1.504(3) \\ \mathrm{C} 3 & \mathrm{C} 4 & 1.341(3) & \mathrm{C} 12 & \mathrm{C} 13 & 1.518(3) \\ \mathrm{C} 3 & \mathrm{O} 2 & 1.365(2) & \mathrm{C} 15 & \mathrm{C} 16 & 1.371(3) \\ \mathrm{C} 4 & \mathrm{C} 5 & 1.426(3) & \mathrm{C} 15 & \mathrm{C} 20 & 1.377(3) \\ \mathrm{C} 5 & \mathrm{C} 8 & 1.394(3) & \mathrm{C} 15 & \mathrm{~N} 1 & 1.415(2) \\ \text { C5 } & \text { C6 } & 1.434(3) & \mathrm{C} 16 & \mathrm{C} 17 & 1.378(3) \\ \text { C6 } & \mathrm{N} 3 & 1.356(2) & \mathrm{C} 17 & \mathrm{C} 18 & 1.365(3) \\ \text { C6 } & \mathrm{C} 7 & 1.420(3) & \mathrm{C} 18 & \mathrm{C} 19 & 1.365(3) \\ \text { C8 } & \mathrm{C} 9 & 1.373(3) & \mathrm{C} 19 & \mathrm{C} 20 & 1.378(3) \\ \text { C9 } & \mathrm{C} 10 & 1.409(3) & \mathrm{N} 1 & \mathrm{~N} 2 & 1.388(2) \\ \text { C9 } & \mathrm{C} 11 & 1.431(3) & & & \end{array}$

Table S5 Bond Angles for Dharmendar 31b.

\begin{tabular}{|c|c|c|c|c|c|c|c|}
\hline \multirow{2}{*}{$\begin{array}{l}\text { Atom } \\
\mathrm{O} 2\end{array}$} & \multirow{2}{*}{$\begin{array}{l}\text { Atom } \\
\mathrm{C} 1\end{array}$} & \multirow{2}{*}{ O1 1 Atom } & \multirow{2}{*}{$\begin{array}{l}\text { Angle } /^{\circ} \\
107.96(17)\end{array}$} & \multicolumn{3}{|c|}{ Atom Atom Atom } & \multirow{2}{*}{$\begin{array}{l}\text { Angle } /^{\circ} \\
110.64(17)\end{array}$} \\
\hline & & & & $\mathrm{N} 2$ & $\mathrm{C} 11$ & C9 & \\
\hline $\mathrm{C} 7$ & $\mathrm{C} 2$ & $\mathrm{O} 1$ & $127.82(19)$ & $\mathrm{N} 2$ & $\mathrm{C} 11$ & $\mathrm{C} 12$ & $121.74(19)$ \\
\hline $\mathrm{C} 7$ & $\mathrm{C} 2$ & $\mathrm{C} 3$ & $122.76(19)$ & C9 & $\mathrm{C} 11$ & $\mathrm{C} 12$ & $127.59(18)$ \\
\hline $\mathrm{O} 1$ & $\mathrm{C} 2$ & $\mathrm{C} 3$ & $109.42(17)$ & $\mathrm{C} 14$ & $\mathrm{C} 12$ & $\mathrm{C} 11$ & $112.11(18)$ \\
\hline $\mathrm{C} 4$ & $\mathrm{C} 3$ & $\mathrm{O} 2$ & $128.45(19)$ & $\mathrm{C} 14$ & $\mathrm{C} 12$ & $\mathrm{C} 13$ & $112.0(2)$ \\
\hline $\mathrm{C} 4$ & $\mathrm{C} 3$ & $\mathrm{C} 2$ & $122.09(19)$ & $\mathrm{C} 11$ & $\mathrm{C} 12$ & $\mathrm{C} 13$ & $110.98(18)$ \\
\hline $\mathrm{O} 2$ & $\mathrm{C} 3$ & $\mathrm{C} 2$ & $109.46(18)$ & $\mathrm{C} 16$ & $\mathrm{C} 15$ & $\mathrm{C} 20$ & $119.20(19)$ \\
\hline C3 & $\mathrm{C} 4$ & $\mathrm{C} 5$ & $118.13(19)$ & $\mathrm{C} 16$ & $\mathrm{C} 15$ & N1 & $119.74(18)$ \\
\hline $\mathrm{C} 8$ & $\mathrm{C} 5$ & $\mathrm{C} 4$ & $122.21(19)$ & $\mathrm{C} 20$ & $\mathrm{C} 15$ & N1 & $121.06(18)$ \\
\hline $\mathrm{C} 8$ & $\mathrm{C} 5$ & C6 & $118.21(18)$ & $\mathrm{C} 15$ & $\mathrm{C} 16$ & $\mathrm{C} 17$ & $120.4(2)$ \\
\hline $\mathrm{C} 4$ & $\mathrm{C} 5$ & C6 & $119.57(18)$ & $\mathrm{C} 18$ & $\mathrm{C} 17$ & $\mathrm{C} 16$ & $120.6(2)$ \\
\hline N3 & C6 & $\mathrm{C} 7$ & $117.02(17)$ & $\mathrm{C} 17$ & $\mathrm{C} 18$ & $\mathrm{C} 19$ & $118.9(2)$ \\
\hline N3 & C6 & $\mathrm{C} 5$ & $123.44(18)$ & $\mathrm{C} 18$ & C19 & $\mathrm{C} 20$ & $121.2(2)$ \\
\hline $\mathrm{C} 7$ & C6 & $\mathrm{C} 5$ & $119.53(18)$ & $\mathrm{C} 15$ & $\mathrm{C} 20$ & C19 & $119.7(2)$ \\
\hline $\mathrm{C} 2$ & $\mathrm{C} 7$ & C6 & $117.91(19)$ & $\mathrm{C} 10$ & N1 & $\mathrm{N} 2$ & $110.14(15)$ \\
\hline C9 & $\mathrm{C} 8$ & $\mathrm{C} 5$ & $119.03(18)$ & $\mathrm{C} 10$ & N1 & $\mathrm{C} 15$ & $130.70(16)$ \\
\hline $\mathrm{C} 8$ & C9 & $\mathrm{C} 10$ & $117.66(18)$ & $\mathrm{N} 2$ & N1 & $\mathrm{C} 15$ & $119.15(15)$ \\
\hline $\mathrm{C} 8$ & C9 & $\mathrm{C} 11$ & $137.16(19)$ & $\mathrm{C} 11$ & $\mathrm{~N} 2$ & N1 & $107.48(16)$ \\
\hline $\mathrm{C} 10$ & C9 & $\mathrm{C} 11$ & $105.15(17)$ & $\mathrm{C} 10$ & N3 & C6 & $114.91(17)$ \\
\hline N3 & $\mathrm{C} 10$ & N1 & $126.68(17)$ & $\mathrm{C} 2$ & $\mathrm{O} 1$ & $\mathrm{C} 1$ & $106.45(16)$ \\
\hline N3 & $\mathrm{C} 10$ & C9 & $126.74(18)$ & $\mathrm{C} 3$ & $\mathrm{O} 2$ & $\mathrm{C} 1$ & $106.57(16)$ \\
\hline N1 & $\mathrm{C} 10$ & C9 & $106.57(16)$ & & & & \\
\hline
\end{tabular}

Table S6 Hydrogen Bonds for Dharmendar 31b.

\begin{tabular}{|c|c|c|c|c|c|c|}
\hline D & $\mathbf{H}$ & $\mathbf{A}$ & $\mathrm{d}(\mathrm{D}-\mathrm{H}) / \AA$ & $\mathbf{d}(\mathbf{H}-\mathbf{A}) / \AA$ & $\mathbf{d}(\mathbf{D}-\mathbf{A}) / \AA$ & D-H-A/ ${ }^{\circ}$ \\
\hline $\mathrm{C} 1$ & H1B & $\mathrm{N} 2^{1}$ & 0.97 & 2.67 & $3.418(3)$ & 134.4 \\
\hline $\mathrm{C} 20$ & $\mathrm{H} 20$ & N3 & 0.93 & 2.34 & $2.979(3)$ & 125.6 \\
\hline $\mathrm{C} 1$ & H1B & $\mathrm{N} 2^{1}$ & 0.97 & 2.67 & $3.418(3)$ & 134.4 \\
\hline $\mathrm{C} 20$ & $\mathrm{H} 20$ & N3 & 0.93 & 2.34 & $2.979(3)$ & 125.6 \\
\hline
\end{tabular}


Table S7 Torsion Angles for Dharmendar 31b.
A $\quad$ B $\quad$ C $\quad$ D
Angle ${ }^{\circ}$
$\begin{array}{llll}A & B & C & \mathbf{D}\end{array}$
Angle $/^{\circ}$
$\begin{array}{llll}\text { C7 } & \text { C2 } & \text { C3 } & \text { C4 } 4\end{array}$
$-0.8(3)$
C9 $\mathrm{C} 11 \mathrm{C} 12 \mathrm{C} 13$
$-64.0(3)$
$\mathrm{O} 1 \mathrm{C}_{2} \mathrm{C} 3 \mathrm{C} 4$
$178.37(19) \quad \mathrm{C} 20 \mathrm{C} 15 \mathrm{C} 16 \mathrm{C} 17$
$0.4(3)$
$\begin{array}{llll}\mathrm{C} 7 & \mathrm{C} 2 & \mathrm{C} 3 & \mathrm{O} 2\end{array}$
9) $\mathrm{N} 1 \mathrm{C} 15 \mathrm{C} 16 \mathrm{C} 17$
$-179.0(2)$
$\begin{array}{llll}\mathrm{O} 1 & \mathrm{C} 2 & \mathrm{C} 3 & \mathrm{O} 2\end{array}$
-0.5 (2) $\mathrm{C} 15 \mathrm{C} 16 \mathrm{C} 17 \mathrm{C} 18$
$0.8(4)$
O2 $\quad$ C3 3445
179.12(19) C16C17C18C19
$-1.0(4)$
C2 $\mathrm{C} 3 \quad \mathrm{C} 4 \mathrm{C} 5$
0.5 (3) C17C18C19C20
0.1 ( 4)
$\begin{array}{llll}\mathrm{C} 3 & \mathrm{C} 4 & \mathrm{C} 5 & \mathrm{C} 8\end{array}$
$178.63(19)^{-} \mathrm{C} 16 \mathrm{C} 15 \mathrm{C} 20 \mathrm{C} 19$
$-1.2(3)$
$\mathrm{C} 3 \mathrm{C} 4 \mathrm{C} 5 \mathrm{C} 6$
0.2 (3) N1 C15C20C19
$178.1(2)$
C8 $\quad$ C5 $\quad$ C6 $\mathrm{N} 3$
-0.8 (3) C18C19C20C15
$1.0(4)$
C4 45 C6 6 N3
8) $\mathrm{N} 3 \mathrm{C} 10 \mathrm{~N} 1 \mathrm{~N} 2$
$\begin{array}{llll}\mathrm{C} 8 & \mathrm{C} 5 & \mathrm{C} 6 & \mathrm{C} 7\end{array}$
$179.69(18)^{-}$
$177.87(17)^{-}$
$\begin{array}{llll}\mathrm{C} 4 & \mathrm{C} 5 & \mathrm{C} 6 & \mathrm{C} 7\end{array}$
$178.29(17)$ C9 C10N1 N2
$1.0(2)$
O1 C2 C7 C6
$\begin{array}{lllll}\mathrm{C} 3 & \mathrm{C} 2 & \mathrm{C} 7 & \mathrm{C} 6\end{array}$
$-0.6(3) \mathrm{N} 3 \mathrm{C} 10 \mathrm{~N} 1 \mathrm{C} 15$
$3.2(3)$
$\begin{array}{llll}\mathrm{N} 3 & \mathrm{C} 6 & \mathrm{C} 7 & \mathrm{C} 2\end{array}$
$178.62(18)$
C9 C10N1 C15
$177.95(18)$
0.4 (3) $\mathrm{C} 16 \mathrm{C} 15 \mathrm{~N} 1 \mathrm{C} 10 \quad 169.2(2)$
C5 $\quad$ C6 $\quad$ C7 $\quad$ C2
$179.46(17)$ C20C15N1 C10
$-10.2(3)$
$0.3(3) \mathrm{C} 16 \mathrm{C} 15 \mathrm{~N} 1 \mathrm{~N} 2 \quad-9.7(3)$
$\begin{array}{lllllllll}\mathrm{C} 4 & \mathrm{C} 5 & \mathrm{C} 8 & \mathrm{C} 9 & 178.76(18) & \mathrm{C} 20 \mathrm{C} 15 \mathrm{~N} 1 & \mathrm{~N} 2 & 170.99(18)\end{array}$
$\begin{array}{lllllllll}\mathrm{C} 6 & \mathrm{C} 5 & \mathrm{C} 8 & \mathrm{C} 9 & -0.1(3) & \mathrm{C} 9 & \mathrm{C} 11 \mathrm{~N} 2 & \mathrm{~N} 1 & -0.4(2)\end{array}$
$\begin{array}{llllllll}\mathrm{C} 5 & \mathrm{C} 8 & \mathrm{C} 9 & \mathrm{C} 10 & 0.8(3) & \mathrm{C} 12 \mathrm{C} 11 \mathrm{~N} 2 & \mathrm{~N} 1 & 177.58(17)\end{array}$

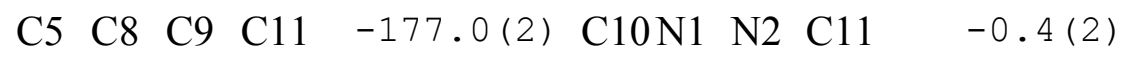

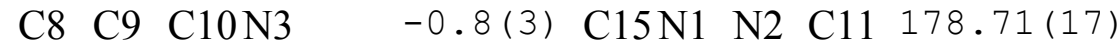
C11C9 C10N3 177.68(18) N1 C10N3 C6 178.55(17)
$\begin{array}{lllllll}\text { C8 C9 C10N1 } & 179.62(1)^{-} & \text {C9 } & \text { C10N3 C6 } & -0.1(3)\end{array}$
$\begin{array}{llllllll}\text { C11C9 C10N1 } & -1.2(2) & \text { C7 } & \text { C6 } & \text { N3 } & \text { C10 } & 178.26(17)\end{array}$

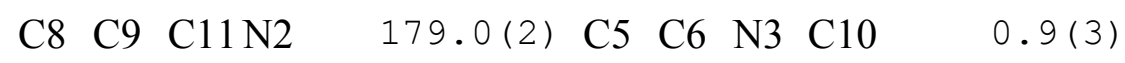
$\begin{array}{lllllll}\mathrm{C} 10 \mathrm{C} 9 & \mathrm{C} 11 \mathrm{~N} 2 & 1.0(2) & \mathrm{C} 7 & \mathrm{C} 2 & \mathrm{O} 1 \mathrm{C} 1 & -178.2(2)\end{array}$
$\begin{array}{llllllll}\mathrm{C} 8 & \mathrm{C} 9 & \mathrm{C} 11 \mathrm{C} 12 & 1.1(4) & \mathrm{C} 3 & \mathrm{C} 2 & \mathrm{O} 1 \mathrm{C} 1 & 2.7(2)\end{array}$
C10C9 C11C12 $176.85(19)^{-} \mathrm{O} 2 \mathrm{C} 1 \mathrm{O}^{2} \mathrm{C} 2 \quad-3.8(2)$
$\begin{array}{llllllll}\mathrm{N} 2 & \mathrm{C} 11 \mathrm{C} 12 \mathrm{C} 14 & -7.7(3) & \mathrm{C} 4 & \mathrm{C} 3 & \mathrm{O} 2 & \mathrm{C} 1 & 179.3(2)\end{array}$

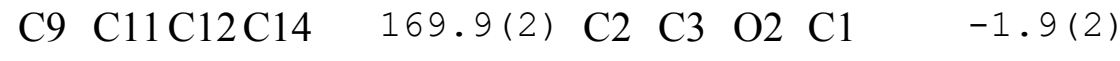

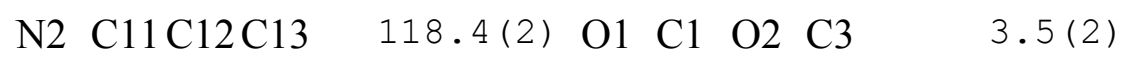

Table S8 Hydrogen Atom Coordinates $\left(\AA \times 10^{4}\right)$ and Isotropic Displacement Parameters $\left(\AA^{2} \times 10^{3}\right)$ for Dharmendar 31b. 


\begin{tabular}{lrrrr} 
H1A & 1917 & 3090.96 & 23.03 & 70 \\
H1B & -48.67 & 3347.48 & 801.75 & 70 \\
H4 & 600.42 & 7064.76 & 2574.86 & 63 \\
H7 & 2954.51 & 2125.1 & 3815.73 & 57 \\
H8 & 1176.7 & 7931.39 & 4440.92 & 57 \\
H12 & 432.81 & 9504.11 & 6906.56 & 66 \\
H13A & 4001.86 & 9142.5 & 5819.8 & 102 \\
H13B & 2301.08 & 10694.99 & 6025.64 & 102 \\
H13C & 2568.26 & 9436.02 & 5085.86 & 102 \\
H14A & 1001.27 & 9116.22 & 8832.04 & 131 \\
H14B & 1289.85 & 10524.32 & 8296.04 & 131 \\
H14C & 2964.41 & 8985.13 & 8245.2 & 131 \\
H16 & 4813.32 & 4873.73 & 8881.14 & 69 \\
H17 & 6193.14 & 2859.63 & 9966.66 & 81 \\
H18 & 6564.47 & 498.7 & 9442.14 & 75 \\
H19 & 5471.24 & 178.83 & 7861.65 & 79 \\
H20 & 4018.46 & 2188.96 & 6798.57 & 70 \\
\hline
\end{tabular}

